\title{
DISCLAIMER:
}

This document does not meet the current format guidelines of the Graduate School at The University of Texas at Austin.

It has been published for informational use only. 
Copyright

by

Youngsung Kwon

2015 
The Dissertation Committee for Youngsung Kwon Certifies that this is the approved version of the following dissertation:

\section{Microgrids for Base Stations: Renewable Energy Prediction and Battery Bank Management for Effective State of Charge Control}

\section{Committee:}

Ross Baldick, Supervisor

Alexis Kwasinski, Co-Supervisor

Aristotle Arapostathis

Edward $\mathrm{Yu}$

Robert Hebner 


\title{
Microgrids for Base Stations: Renewable Energy Prediction and Battery Bank Management for Effective State of Charge Control
}

by

Youngsung Kwon, B.S.E.; M.S.E.

\author{
Dissertation \\ Presented to the Faculty of the Graduate School of \\ The University of Texas at Austin \\ in Partial Fulfillment \\ of the Requirements \\ for the Degree of \\ Doctor of Philosophy
}

The University of Texas at Austin

December 2015 


\section{Dedication}

This dissertation is dedicated to my lovely wife Raehee Park, my sons Daniel Yoobin Kwon and Joseph Kyubin Kwon, and, my parents Myungsik Kwon and Aewall Kang. 


\section{Acknowledgements}

First and foremost, I would like to express the deepest appreciation to my $\mathrm{Ph} . \mathrm{D}$ advisor and supervisor Professor Kwasinski for not only teaching me technical contents, but also helping me to develop my reasoning and learning skills throughout my doctoral studies. Whenever I struggled in academic difficulties, he always guided me in the right direction and believed in me to develop my own ideas continuously. Moreover, whenever I faced personal problems, he was always there with a sympathetic ear. I really would like to thank his support. I would also like to thank him for providing me documentation and ideas in order to support and improve my research method. I would like to thank Professor Baldick for his continuous encouragement and suggestions for my doctoral studies. Particularly, his passion and kindness inspired me to successfully complete my dissertation. I would especially like to thank Professor Andres Kwasinski for guiding me to the field of communication system so that I could complete this research. I was lucky to collaborate with him during my doctoral study. I would also like to thank my master's degree supervisor Professor W. Mack Grady who inspired me to have a broad knowledge of renewable energy systems.

Besides my supervisors, I would like to express my gratitude to the distinguished members who served on my Ph.D dissertation committee, Professor Arapostathis, Professor Yu, and Dr. Hebner at the Center for Electromechanics. I cannot forget their valuable comments and support that made me to successfully complete this work.

I would also like to thank the National Science Foundation for providing the grant to conduct my research.

My thanks also go to my friends, Jin Hur, Seunghoon Choung, Seunghyun Chun,

Cheolhee Cho, Wonjin Cho, Heejung Park, Sungwoo Bae, Juyoung Jung, Jihoon Yoon, 
Duhee Lee, Junseok Song, Myungchin Kim, Joohyun Jin, Joonhyun Kim, Han Kang, Hoonyoung Shin, Kyungwoo Min, Wanki Cho, Vaidyanathan Krishnamurthy, Harsha Kumar, Amir Toliyat, Mahesh Srinivasan, Rossen Tsartzev, and Moses Kai. I would especially like to thank Vaidyanathan for his critical feedback in research.

A very special thanks goes to Austin Korean Presbyterian Church and Oryu-dong Nambu Church for their continuous prayers for not only my studies, but also the local communities and all over the world. Especially, I would like to thank my old church friends Cheol Hong, Soomin Kim, Sanghun Park, Sanghun Kim, Kyungho Kim, Jinhee Lee, Yukyung Lee, Kayoung Lee, Jiyoung Park, Sunhwa Lee, and Minsun Kim.

Last, but not the least, I would like to thank my family, my grandmother Soonboon Kim, my father Myungsik Kwon, my mother Aewall Kang, and my sister Hanna Kwon for their persistent encouragement, support, love, and pray me through studying abroad in USA. Also, I want to thank my parents-in-law Haekyung Park and Mikyung Yu, my sisterin-law, Raeyoung Park and Raejin Park and my brother-in-law Raechan Park for their loving and prying. Especially, I am deeply indebted to my lovely wife Raehee Park for her limitless love and belief in me. Without their support and pray, this dissertation would not have been possible.

Youngsung Kwon

The University of Texas at Austin

December 2015 


\title{
Microgrids for Base Stations: Renewable Energy Prediction and Battery Bank Management for Effective State of Charge Control
}

\author{
Youngsung Kwon, Ph.D. \\ The University of Texas at Austin, 2015 \\ Supervisor: Ross Baldick \\ Co-Supervisor: Alexis Kwasinski
}

Power consumption of cellular communication infrastructure has been considered as a global issue due to its exceptional growth rate. In order to address the challenges posed by the increasing power consumption such as carbon emissions, many studies have been focused on designing green cellular networks where carbon emission can be mitigated by reducing power consumption at base stations. Although the previous studies have a common theme of the power saving strategies at base stations, most studies develop the power saving techniques are restricted to cases where the electricity is supplied from conventional grid instead of renewable energy sources.

The use of renewable sources poses two main issues such as large footprint and power output variability due to the variation of the natural phenomena driving the sources. This research thus aims at addressing the cellular networks power consumption issue by considering a cluster of neighboring base station in a microgrid configuration, called sustainable wireless area (SWA) that can not only reduce the carbon emission effectively by being powered from distributed renewable sources, but also overcome the renewable power output variability issue by using local energy storage and renewable power 
prediction two-days. In this sense, this dissertation investigates a novel approach to realize sustainable power supply in a SWA, which incorporates the design of renewable sources, energy storage, and base station electric architecture, and a power control algorithm for their operation.

With the aim of mostly powering base stations from renewable sources, the power control strategies are explored based on the predicted renewable energy and battery bank state of charge (SOC). Finally, the effectiveness of the control strategies is evaluated regarding on SWA resiliency and battery bank lifetime aspects. 


\section{Table of Contents}

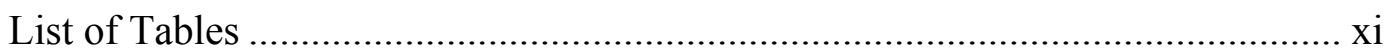

List of Figures ..................................................................................

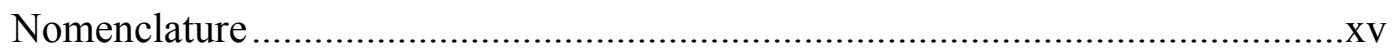

Chapter 1. Introduction .............................................................................1

1.1 Research Motivation .....................................................................

1.2 Literature Review......................................................................2

1.3 Base Stations in A Microgrid Configuration ..........................................5

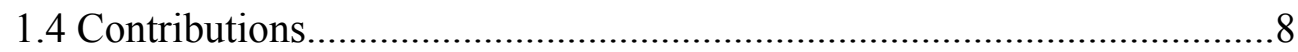

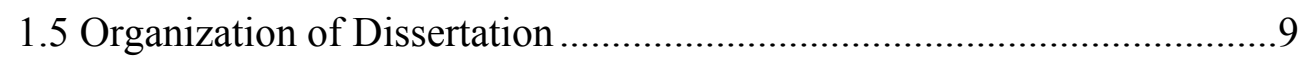

Chapter 2. Power Output Model ...........................................................................11

2.1 Data Description ............................................................................ 11

2.1.1 Weather Variables............................................................ 12

2.1.2 Solar Irradiance ............................................................... 13

2.2 Renewable Source Model .............................................................. 16

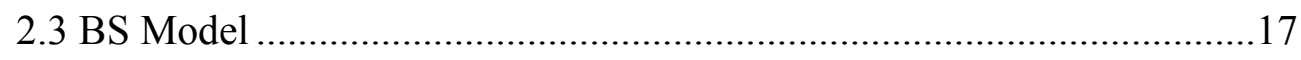

Chapter 3. Renewable Energy Prediction Model..........................................22

3.1 Literature Review.......................................................................22

3.2 Solar Power Prediction Model ..........................................................25

3.2.1 Step 1: Deterministic Characteristic of GHI ..........................25

3.2.2 Step 2: GHI Variation by Clouds.........................................27

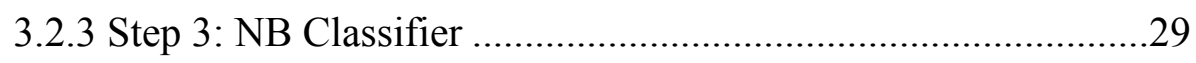

3.2.4 Estimation of $\mathrm{P}\left(\mathrm{V}_{\mathrm{i}} \mid \mathrm{C}\right)$ : Kernel Method .................................32

3.2.5 Update Posterior of $\mathrm{C}$ : Calculating the Value of $\mathrm{C} \mid \mathrm{V}_{\mathrm{i}} \ldots \ldots \ldots \ldots . . . .34$

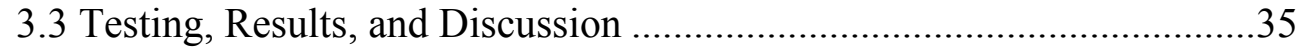

3.3.1 Diagnostic Checking ......................................................... 35

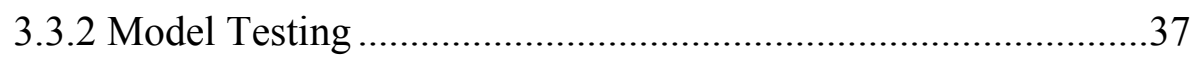

3.4 Wind Power Prediction Model...........................................................45 
Chapter 4. Battery SOC Estimation...................................................................47

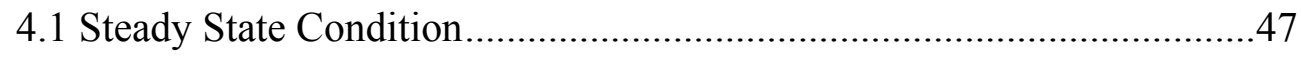

4.1.1 One-Step Tranistion Probability Matrix ……………………........47

4.1.2 Limiting Probabilities ...................................................................49

4.1.3 Availability vs Capacity ............................................................ 51

4.1.4 Statistical Test for Markove Chain Model....................................51

4.1.5 Sizing Battery Bank (Energy Storage)........................................53

4.2 Real Time Condition............................................................................. 61

Chapter 5. Integrated Power Management in SWA............................................64

5.1 Sustainable Wireless Area (SWA) ........................................................64

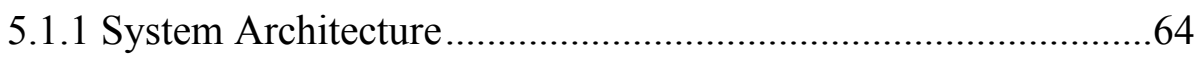

5.1.2 High Level Controller ...............................................................66

5.2 Integrated Traffic-Power Control .......................................................67

5.2.1 Traffic Shaping Strategies........................................................68

5.2.2 Power Sharing Strategies ...........................................................71

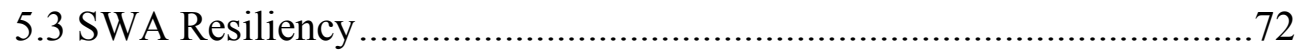

5.4 Battery Bank Lifetime Model .............................................................

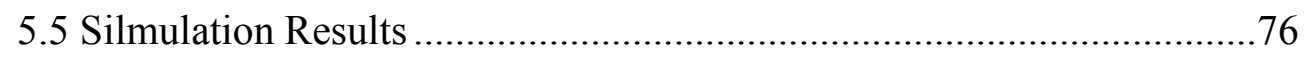

Chapter 6. Conclusions......................................................................................85

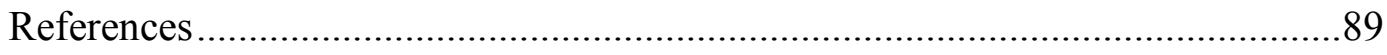

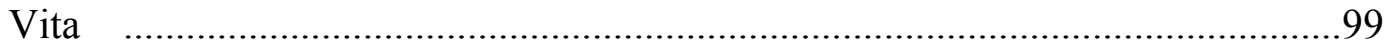




\section{List of Tables}

Table 1.1: Worldwide ICT power conumption ......................................................

Table 3.1: Summary of statistical values for actual GHI and prediction errors ....39

Table 3.2: Summary of two days ahead prediction errors for 4 days .....................44

Table 3.3: Summary of one day ahead prediction errors for 4 days .......................44

Table 4.1: The proposed Markov chain dependency and stationary test for two

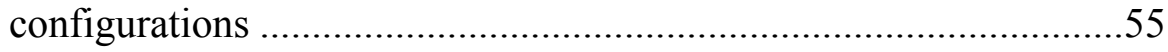

Table 4.2: Statistics of predicting renewable energy in two configurations..........63

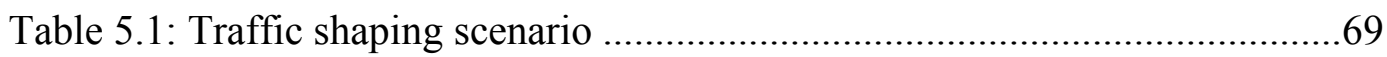

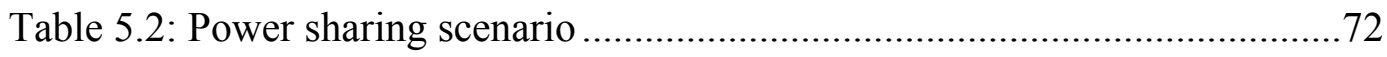

Table 5.3: Comparison between no traffic shaping and traffic sharing

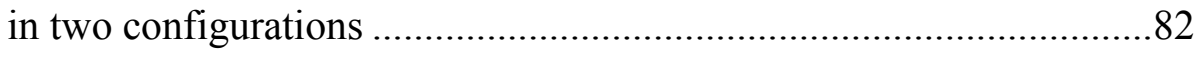

Table 5.4: SOC results for power sharing in SWA.............................................83

Table 5.5: Comparison between local traffic shaping and power sharing in two configuration .84 


\section{List of Figures}

Fig. 1.1: Worldwide electricity consumption prediction of ICT ….....................2

Fig. 1.2: Wind turbine applications which are found in a centralized location in a microgrid configuration ..............................................................

Fig. 1.3: PV applications for powering BSs ................................................

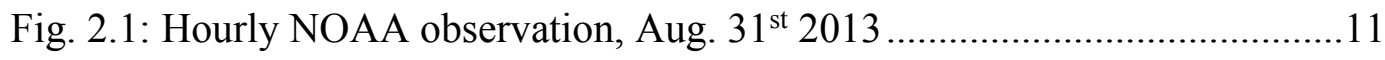

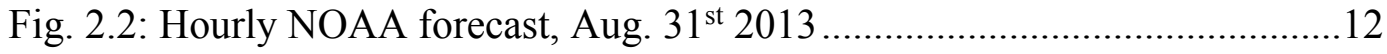

Fig. 2.3: 8 months solar irradiance data set used in NB model:(a) hourly actual GHI, (b) normalization factor ESR, and (c) clear index $k t . .$.

Fig. 2.4: Monthly average energy production in two configurations from August 2013 to March 2014. ................................................................17

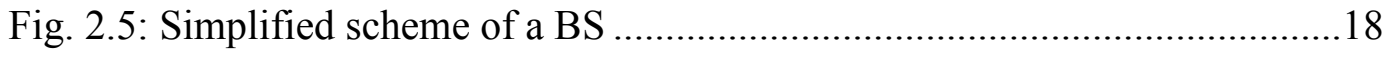

Fig. 2.6: BS power consumtion vs traffic load ................................................19

Fig. 2.7: Normalized hourly average traffic profile in Europe ............................21

Fig. 3.1: Deterministic characteristic of GHI on a clear day and an overcast day.26

Fig. 3.2: The fourteen one-hour observation training sets obtained from separation of the daylight data (step 1) ...........................................27

Fig. 3.3: Filtered training set using the forecasted sky coverage variable (step 2)28

Fig. 3.4: Modified NB classfication using forecasted weather variables (step 3) .30

Fig. 3.5: Comparison between actual and predicted GHI for 8 months:

(a) scatter plot and (b) cumulative distribution function .40

Fig. 3.6: Comparison between two days ahead predicted and actual GHI for clear 4 days (type 1 )

Fig. 3.7: Comparison between two days ahead predicted and actual GHI 
for 2 clear, 1 partly cloudy, and 1 overcast day (type 2)

Fig. 3.8: Comparison between two days ahead predicted and actual GHI for 3 overcast and 1 clear day (type 3 ).

Fig. 3.9: Comparison between two days ahead predicted and actual GHI for 1 clear and 3 partly cloudy days (type 4$)$

Fig. 4.1: State transition diagram for the battery bank SOC.

Fig. 4.2: Data time line: Power supply availability evaluation (red) and real-time operation period (green) 54

Fig. 4.3: 8.1 kWh battery bank capacity with 24 SOC states ............................56

Fig. 4.4: $9.45 \mathrm{kWh}$ battery bank capacity with 28 SOC states ...........................56

Fig. 4.5: Sizing battery capacity (SOC) depending on target power supply availabilty 58

Fig. 4.6: Two configurations' cost analysis with different battery bank cost 59

Fig. 4.7: Sizing battery bank capacity (SOC) with traffic shaping that is considered as a long-term process $(\sigma=0.9$ for $\mathrm{C} 1$ and 0.5 for $\mathrm{C} 2)$ 60

Fig. 5.1: Proposed SWA architecture that comprises of seven base stations with renewable source and storage system .65

Fig. 5.2: Electrical scheme of a SWA .............................................................66

Fig. 5.3: Hierarchical controller architecture ..............................................67

Fig. 5.4: A real-time iteration algorithm using Markov chain .............................69

Fig. 5.5: One day results for trrfic shaping strategies in Sep. $4^{\text {th }} 2013$ :

(a) SOC, (b) traffic shaping factor, and (c) actual and predicted renewable energy .70

Fig. 5.6: Expected life cycle \& throughput vs DOD for deep-cycle battery model for US 250E XC2 
Fig. 5.7: Cumulative distribution functions (CDFs) for the battery SOC with different operating conditions for 8 months:

configuration 1 (a) and configuration 2 (b).......................................78

Fig. 5.8: Percentage of three level of SOC with different operating conditions for 8 months: configuration 1 (a) and configuration 2 (b) .80

Fig. 5.9: Cumulative distribution functions (CDFs) for the power sharing in SWA 


\section{Nomenclature}

\begin{tabular}{|c|c|}
\hline SWA & Sustainable wireless area. \\
\hline $\mathrm{SOC}$ & State of charge. \\
\hline $\mathrm{ICT}$ & Information and communication technologies. \\
\hline QOS & Quality of service. \\
\hline $\mathrm{BS}$ & Base station. \\
\hline GHI & Global horizontal irradiance. \\
\hline $\mathrm{CI}$ & Critical infrastructure. \\
\hline ESR & Extraterrestrial solar radiation. \\
\hline NB classifier & Naïve Bayes classifier. \\
\hline ANN & Artificial neural networks. \\
\hline MLP & Multilayer perceptron. \\
\hline MBE & Mean bias error. \\
\hline RMBE & Relative mean bias error. \\
\hline RMSE & Root mean square error. \\
\hline RRMSE & Relative root mean square error. \\
\hline MAPE & Mean absolute percentage error. \\
\hline LOLP & Loss of load probability. \\
\hline DOD & Depth of discharge. \\
\hline
\end{tabular}




$\begin{array}{ll}\text { MUT } & \text { Mean up time. } \\ \text { MDT } & \text { Mean down time. } \\ \text { QOE } & \text { Quality of experience. } \\ \text { CTF } & \text { Cycles to failure. } \\ \text { CDF } & \text { Cumulative distribution function. } \\ \text { C1 } & \text { Configuration } 1 . \\ \text { C2 } & \text { Configuration } 2 .\end{array}$




\section{Chapter 1. Introduction}

\subsection{RESEARCH Motivation}

In recent years, power consumption of information and communication technologies (ICT) have been considered as global issues due to their rapid global growth rate. Recent studies [1], [2] expect a potential continuous and significant growth in the future, which is reflected in past and present growing tendency: from the average power consumption of $168 \mathrm{GW}$ in year 2008 to the predicted power consumption of $433 \mathrm{GW}$ in year 2020 (about 2.58 times) as shown in Table 1.1 and Fig. 1.1. This trend shows the exceptional growth rate of electric consumption itself, but it is more remarkable when it is compared with a worldwide electricity consumption of $2350 \mathrm{GW}$ in year 2008 to $2970 \mathrm{GW}$ in year 2020 (about 1.26 times).

\begin{tabular}{|l|c|c|}
\hline Type & \multicolumn{1}{|c|}{$\begin{array}{c}\text { Year 2008 } \\
\text { Power } \\
\text { consumption [GW] }\end{array}$} & $\begin{array}{c}\text { Year 2020 } \\
\text { Predicted power } \\
\text { consumption [GW] }\end{array}$ \\
\hline Data centers & 29 & 113 \\
\hline $\begin{array}{l}\text { Network } \\
\text { Equipment }\end{array}$ & 25 & 97 \\
\hline TVs & 44 & 79 \\
\hline PCs & 30 & 71 \\
\hline Other & 40 & 72 \\
\hline Total & 168 & 433 \\
\hline \multicolumn{2}{|l|}{} \\
\hline $\begin{array}{l}\text { Worldwide } \\
\text { Electricity }\end{array}$ & 2350 & 2970 \\
\hline ICT fraction & $7.15 \%$ & $14.57 \%$ \\
\hline
\end{tabular}

Table 1.1: Worldwide ICT Power Consumption. 


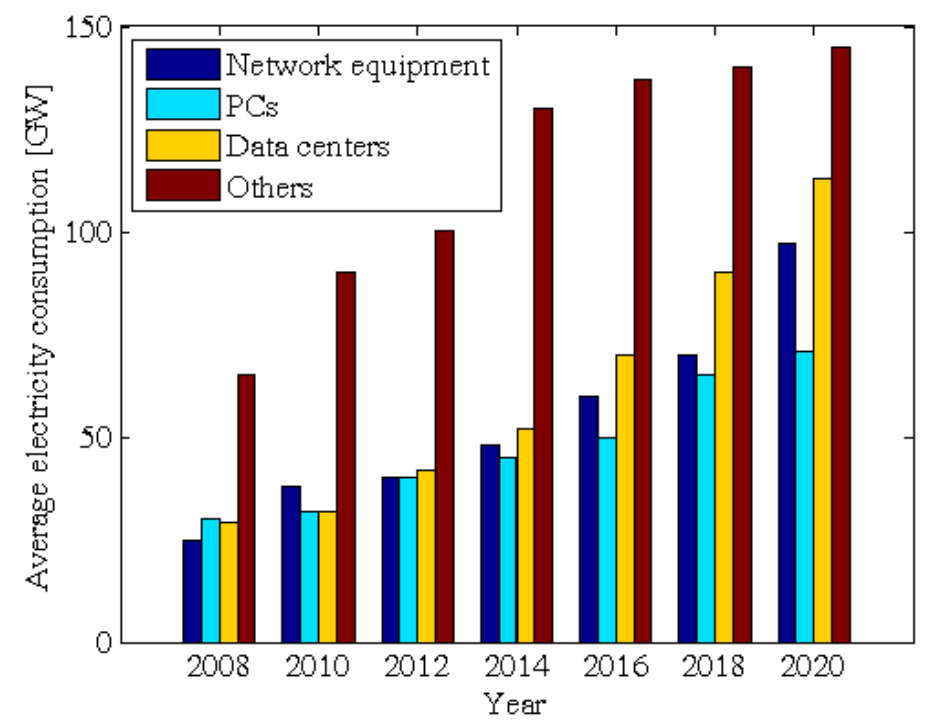

Fig. 1.1: Worldwide electricity consumption prediction of ICT.

Among the ICT infrastructure, telecommunication networks, which account for about one sixth power demand have been researched in order to develop power saving strategies for the operation equipment [3]. Within cellular networks, to be more concrete, a considerable increase in a decade (from 700 million users in year 2000 to 5.9 billion users in year 2011) anticipates a continuous growth in the near future [4]. With the increase of power consumption, carbon dioxide $\left(\mathrm{CO}_{2}\right)$ emissions and grid reliability have been pointed out as the main challenges in the cellular network area.

\subsection{LiterATURE REVIEW}

In the past, most studies in cellular network focused on solutions that improve the Quality of Service (QOS) and availability corresponding to the rapid growth of mobile 
users. As environmental and network resilience concerns rise researchers have increasingly been interested in designing "green" cellular networks in which energy-efficiency system architectures and operation algorithms can be designed to ease environmental concerns [5]. To end this, the research focus has turned to the operation of base stations because they are attributed to about $60 \%$ of the energy consumed in cellular networks [6], [7]. Although the related literature has the common subject of optimal operation aspects, the associated problems are dealt from different points of view for the power saving strategies. The studies in [8] and [9] present an approach, which is to switching off (sleeping mode) a one selected base stations (BSs) according to traffic demand condition in cellular networks. Also, the study [10] proposes an efficient cell zooming technique that can adjust cell size in order to balance traffic load and reduce energy consumption. Another study in [11] suggests various techniques such as interference management (IM) and resource management by power control. These techniques might be helpful in reducing $\mathrm{CO}_{2}$, which are effective since the existing methods are powered from conventional grid or diesel generators. However, the mitigating effects are less when renewable sources are considered in cellular network for powering BSs.

Renewable energy, such as photovoltaics (PVs) and wind turbine, might be a better alternative means for power saving and reduction of $\mathrm{CO}_{2}$ emission in the cellular networks. Renewable energy is desirable for its independence from conventional grid and allows avoiding the uses of fossil fuel reserves, thus aiding in mitigating $\mathrm{CO}_{2}$ emission [12]. In remote areas where conventional grid cannot be reached, renewable energy allows avoidance of huge capital investment for installing grid infrastructure or fuel delivery costs 
for operating diesel generators [13]. The studies [14], [15] propose renewable energy installations as main power sources for BS so that $\mathrm{CO}_{2}$ emission would be effectively mitigated in cellular networks. In [14], power management policies among LTE BSs are developed using online and offline algorithm with stochastic and deterministic energy profile in order to minimize the use of conventional sources. In [15], the study focuses on dimensioning renewable sources and energy storage (battery) for a BS operation while preventing excessive battery discharging. However, as the number of renewable sources increases, system planners would be concerned about space allocation due to its large footprint. For example, a general PV module's power density $\left(250 \mathrm{~W} / \mathrm{m}^{2}\right)$ is relatively small when compared to a typical BS $\left(5 \mathrm{~kW} / \mathrm{m}^{2}\right)$. This indicates a footprint that is 20 times larger [16].

More importantly, system operators would be concerned about the generated power output variability, which may cause BSs low power supply availability. Power output variability depends on numerous variables that can be roughly categorized into two main components: 1) systematic effect and 2) natural environmental elements. The former category includes inverter performance and the arrangement or size of the PV arrays'. The latter category includes dust accumulation, precipitation, temperature, and cloud movement. The highest number of disturbances occurs when clouds pass over the PV arrays caused by changes in irradiance. Because of this, many studies have focused on the variability of PV system's output power. The study in [17] analyzed the impact of the power output variability on the various levels of PV penetration and gave an alternative to mitigate the negative effects on the grid. Other studies in [18]-[20] estimated the output 
variability in situations, where PV systems were dispersed over a wide area ranging from a few $\mathrm{km}$ to hundreds of $\mathrm{km}$ in distance. From a relationship between distance and time intervals, correlation factors were calculated and the system's output variability was estimated. These studies evaluated the smoothing effect of wide-spread PV systems. There have also been several studies of energy storage models. In [21], Bucciarelli illustrated battery energy transition through a fundamental model, in which only one level of energy transition was used to represent the charge and discharge process. In [22], the energy transition states were developed with more possible transition probabilities in order to more closely mimic situations that would likely occur in real energy storage systems. Although an energy storage can assist in supplying more sustainable power against the solar and wind power variability [23], [24], in order to improve reliability, it is still necessary to estimate the variability impact on storage models at different time scales that depend on application types [25].

\subsection{BSS IN A Microgrid CONFIguration}

In order to address the issues outlined above ( $\mathrm{CO}_{2}$ emission and reliability) more effectively, the cellular network sites are considered as a microgrid configuration. Microgrids are comprised of local distributed generators with energy storage system and loads, which have been proposed in recent literature as promising power supply solutions for critical loads [26]. Due to this, microgirds are required to be designed and operated optimally in order to have sustainable power supply availability [27], [28]. For example, 
the critical loads such as telecommunication facilities require over 5 nines (0.99999) availability [29]. Moreover, two major drawbacks in conventional grids such as aging and distinct entities namely, generation, transmission, and distribution, spread over large geographic areas, which lead to an increased incidence of weather-related power outages support the necessity of microgrids for the critical loads. The studies in [30], [31] reveal how recent natural disasters affect the wireless communication infrastructure that is powered from conventional grid and the needs of the distributed renewable energy systems are observed in telecommunication networks [32].

Fig. 1.2 and 1.3 (provided by A. Kwasinski) show several examples where the renewable energy and base station in an urban or suburban residential area can be potential applications based on a microgrid. Fig. 1.2 represents two types of horizontal and vertical axis wind turbine that are available in a microgrid configuration. The horizontal wind turbine "Fuhrlander FL 250" is currently used for powering a plastic molding company. As shown in Fig. 1.2, the height of the FL 250 wind turbine is similar to a cell site tower which is located approximately 400 meter from the FL 250. If a residential urban location would be expected to have smaller wind turbines due to space limit, a typical installation can be used such as a vertical axis turbine as shown in Fig. 1.2. Moreover, Fig. 1.3 shows the sufficient space for installing base stations and PV arrays. 


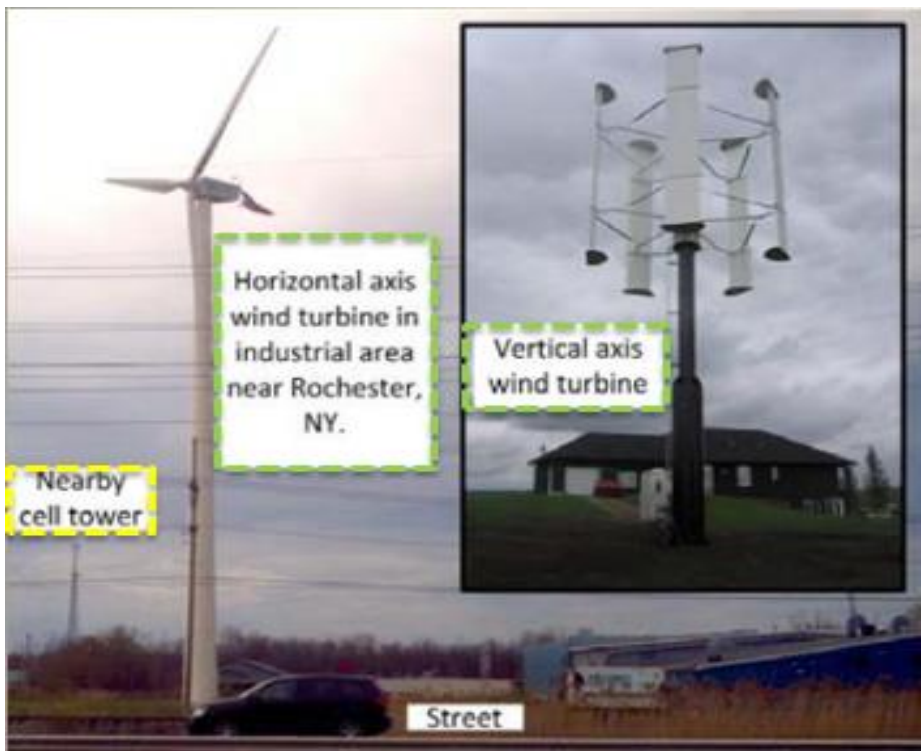

Fig. 1.2: Wind turbine applications which are found in a centralized location in a microgird configuration.
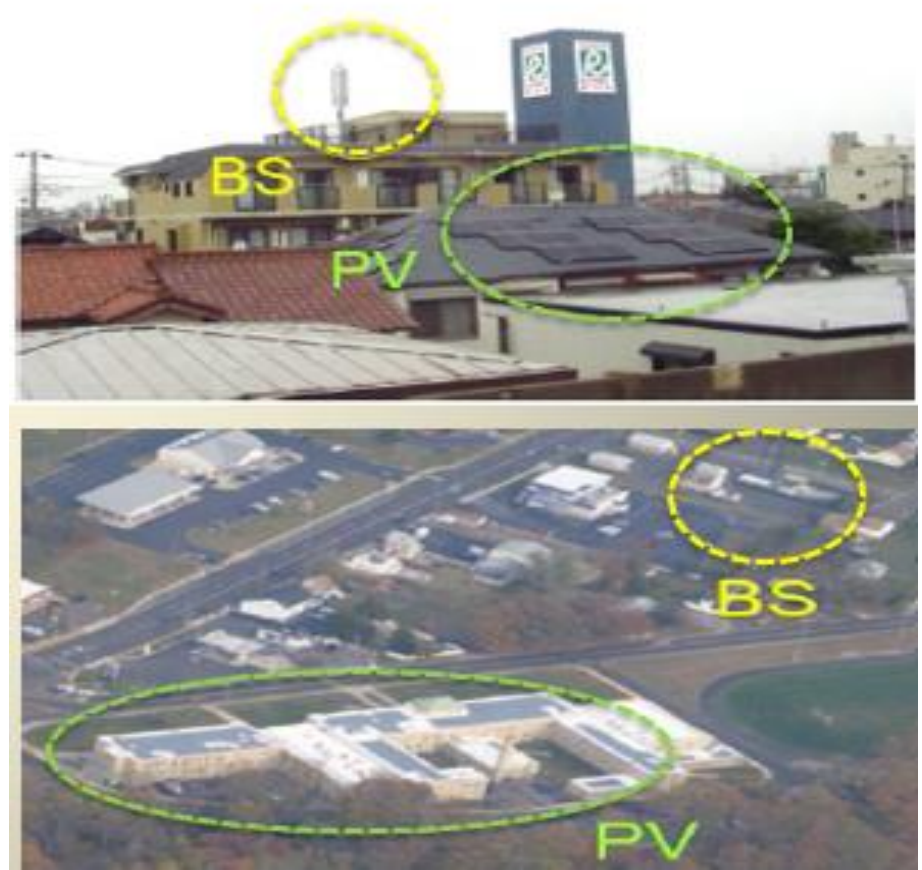

Fig. 1.3: PV applications for powering BSs. 


\subsection{CONTRibutions}

First, this dissertation proposes modeling renewable sources (solar and wind power), which can predict available energy over the following 48 hours. Based on the hourly forecasted and observed weather information at the National Oceanic and Atmospheric Administration (NOAA) [33], global horizontal irradiance (GHI) and wind speed are predicted with naïve Bayes (NB) classifier. A key contribution of the proposed NB model is to reduce the error in the prediction of solar irradiance variability using the five sky coverage states and partitioning daily hours into subsets by an hour. The proposed model is fairly simple in that it requires small training data (less than two months) and uses only four weather variables (temperature, relative humidity, dew point, and sky coverage), whereas the prediction accuracy shows improved results compared to the recent other related models. In contrast with the other models, moreover, the proposed NB model provides hourly GHI prediction as well as a daily total GHI without the effect of the previous predicted results (statistics-based results will be discussed in more detail in Chapter 3). Predicting daily or hourly renewable energy requires GHI or wind speed data that is modeled accurately for designers who need to optimally size the renewable systems before installation, and it might be helpful to users to indicate whether the system is operating efficiently and economically or not.

Second, this dissertation proposes the battery state of charge (SOC) estimation technique that is a key feature in terms of increasing battery life and preventing battery failure [34]-[38]. Different to the existing studies, which measure battery voltage and current in order to estimate battery SOC [34], [35], state of health (SOH) [36], or battery 
life [37], [38], this dissertation uses stochastic Markov chain based on the proposed NB model for the real-time battery SOC estimation. Since the NB model shows a good agreement on mean bias error (MBE) metric, which represents over-predicted or underpredicted tendency between actual and predicted values given the test period, the predicted renewable energy is used to estimate battery SOC. Then this SOC information is used to control the battery operation in cellular works in order to increase availability and prevent the battery deep discharge.

Finally, this dissertation is intended to develop a novel approach that integrates power, computing, and communication system knowledge using the existing models. Therefore, this novel approach can benefit industries, where the integrated operation knowledge currently has been requested such as smart grids and critical infrastructures (CIs) (data centers [39] and military bases [40]).

\subsection{ORgANIZATION OF DiSSERTATION}

This dissertation is organized as follows. Chapter 2 introduces the power output models such as renewable energy (solar and wind) and BS with the actual measurement data. Chapter 3 introduces the naïve Bayes (NB) classifier in order to predict renewable energy. The performance is evaluated and compared with various statistics values and other recent models. More importantly, the properties of proposed NB model is discussed that can be appropriate for renewable energy storage system. Chapter 4 discusses battery SOC estimation with steady-state and real-time condition. The former one is used to size a battery capacity in order to avoid over-sized capacity and the latter is used to control the

battery operation in order to increase availability. Chapter 5 investigates the effectiveness 
of local and global operation in cellular networks from the two points of view of resiliency and battery life. Finally, Chapter 6 concludes the dissertation. 


\section{Chapter 2. Power Output Model}

\subsection{DATA DESCRIPTION}

The hourly weather and GHI data set was obtained at the city of Austin, Texas, USA, from January 2013 to March 2014. GHI represents the total solar irradiation from the entire sky on a horizontal surface, which includes the sum of the direct-beam and diffuse and reflected solar radiation [41]. The weather data were taken from the publicly available website NOAA [33]. Two types of weather data were collected daily: hourly observation as shown in Fig. 2.1 and two day ahead forecast data as shown in Fig. 2.2. Austin typically indicates a warm humid temperature climate with hot summer and no dry season [42].

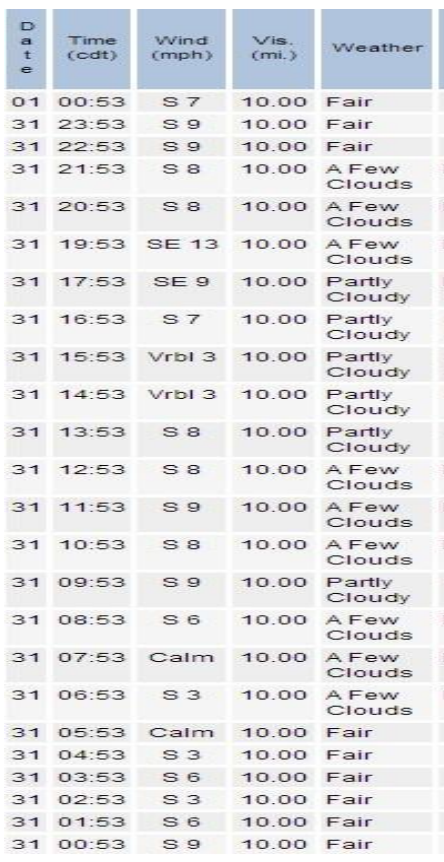

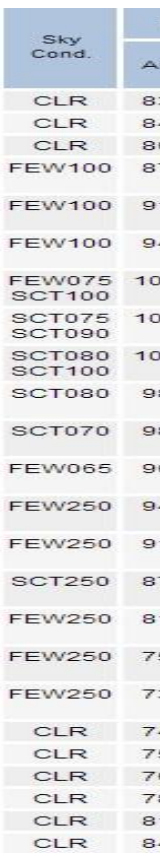

\begin{tabular}{|c|c|c|c|c|}
\hline \multicolumn{3}{|c|}{ Temperature ( $\left.{ }^{\circ} \mathrm{F}\right)$} & \multirow{3}{*}{$\begin{array}{l}\text { Relative } \\
\text { Humidity }\end{array}$} & \multirow{3}{*}{$\begin{array}{l}\text { Wind } \\
\text { Chill } \\
(=F)\end{array}$} \\
\hline \multirow{2}{*}{ Dwpt } & \multicolumn{2}{|c|}{6 hour } & & \\
\hline & $\max$. & Min. & & \\
\hline 68 & 97 & 83 & 6196 & NA \\
\hline 68 & & & $59 \%$ & NA \\
\hline 67 & & & 5396 & NA \\
\hline 66 & & & $50 \%$ & NA \\
\hline 66 & & & 4496 & NA \\
\hline 66 & & & 4096 & NA \\
\hline 65 & & & $32 \%$ & NA \\
\hline 65 & & & $32 \%$ & NA \\
\hline 65 & & & $32 \%$ & NA \\
\hline 65 & & & $34 \%$ & NA \\
\hline 66 & & & $35 \%$ & NA \\
\hline 67 & 97 & 73 & 3996 & NA \\
\hline 67 & & & 4196 & NA \\
\hline 68 & & & 4796 & NA \\
\hline 70 & & & 579 & NA \\
\hline 70 & & & 6996 & NA \\
\hline 69 & & & $82 \%$ & NA \\
\hline 68 & 83 & 73 & $84 \%$ & NA \\
\hline 68 & & & $82 \%$ & NA \\
\hline 68 & & & 7996 & NA \\
\hline 68 & & & 7796 & NA \\
\hline 68 & & & $71 \%$ & NA \\
\hline 68 & & & $65 \%$ & NA \\
\hline 68 & 99 & 84 & $59 \%$ & NA \\
\hline
\end{tabular}

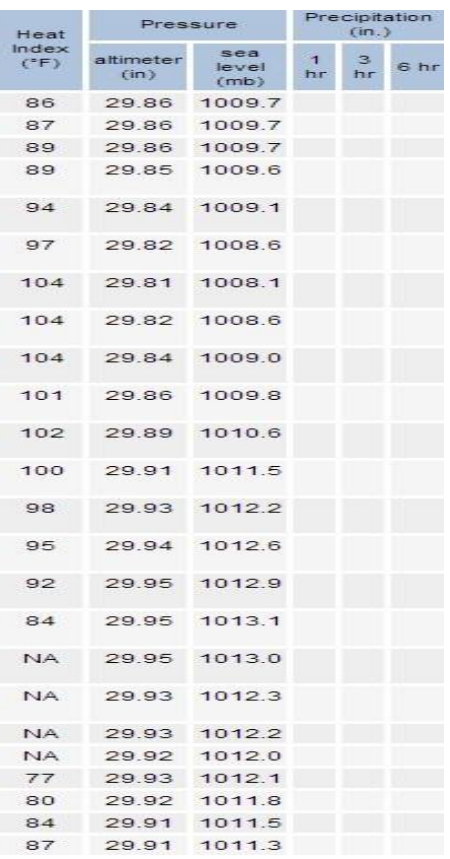

Fig. 2.1: Hourly NOAA observation, Aug. 31 2013. 


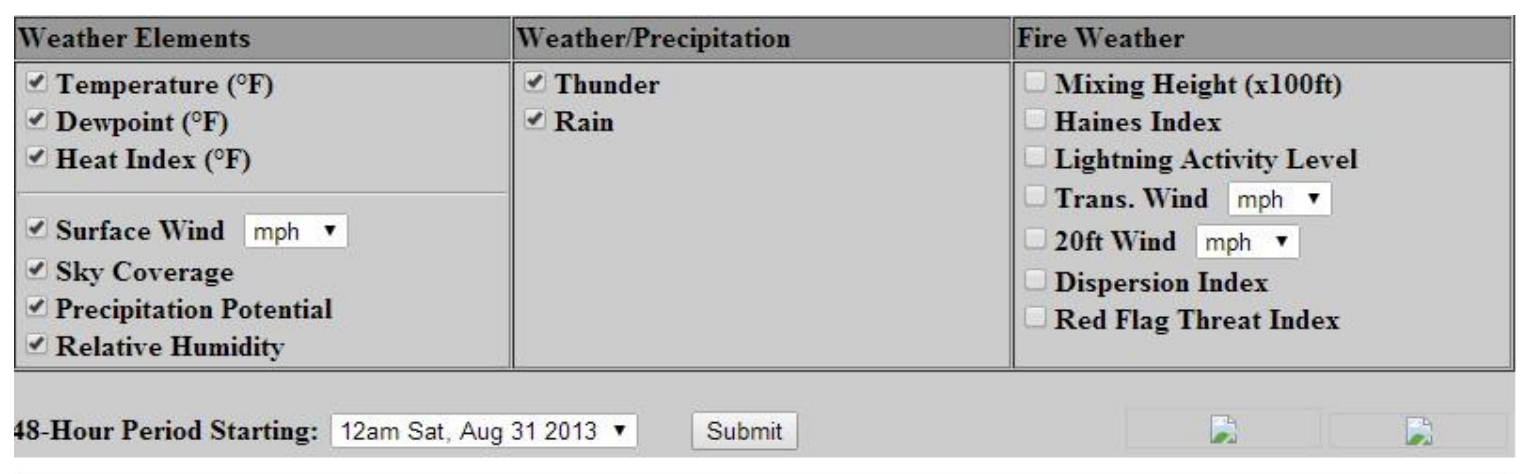

\begin{tabular}{|c|c|c|c|c|c|c|c|c|c|c|c|c|c|c|c|c|c|c|c|c|c|c|c|c|}
\hline Date & $08 / 31$ & & & & & & & & & & & & & & & & & & & & & & & \\
\hline Hour (CDT) & 00 & 01 & 02 & 03 & 04 & 05 & 06 & 07 & 08 & 09 & 10 & 11 & 12 & 13 & 14 & 15 & 16 & 17 & 18 & 19 & 20 & 21 & 22 & 23 \\
\hline $\begin{array}{l}\text { Temperatuce } \\
\text { ("F) }\end{array}$ & 84 & 82 & 82 & 80 & 79 & 78 & 78 & 77 & 79 & 82 & 86 & 90 & 93 & 96 & 98 & 100 & 101 & 100 & 99 & 97 & 94 & 90 & 87 & 86 \\
\hline Derrpoint ( $(\mathrm{F}$ ) & 67 & 68 & 68 & 69 & 70 & 70 & 70 & 69 & 69 & 69 & 68 & 67 & 66 & 65 & 65 & 64 & 64 & 64 & 64 & 64 & 64 & 66 & 66 & 67 \\
\hline $\begin{array}{l}\text { Heat Index } \\
\text { ("F) }\end{array}$ & 87 & 85 & 84 & 83 & 82 & 78 & 78 & 77 & 79 & 85 & 88 & 92 & 96 & 100 & 102 & 104 & 105 & 104 & 102 & 100 & 96 & 92 & 89 & 87 \\
\hline Wind (mph) & 9 & 9 & 9 & 8 & 8 & 7 & 6 & 6 & 7 & 8 & 9 & 8 & 6 & 5 & 5 & 5 & 6 & 7 & 8 & 8 & 10 & 11 & 11 & 13 \\
\hline Wind Dir & $\mathbf{s}$ & $\mathbf{s}$ & $\mathrm{s}$ & s & ssw & ssw & Ssw & Ssw & Ssw & SSw & sw & Ssw & SSW & $\mathrm{s}$ & s & s & SSE & SSE & SSE & SSE & SSE & SSE & SSE & $\mathbf{s}$ \\
\hline \multicolumn{25}{|l|}{ Grost } \\
\hline $\begin{array}{l}\text { Sky Cover } \\
\text { (\%) }\end{array}$ & 5 & 6 & 6 & 6 & 7 & 7 & 7 & 11 & 11 & 11 & 19 & 19 & 19 & 19 & 19 & 19 & 20 & 20 & 20 & 22 & 22 & 22 & 24 & 24 \\
\hline $\begin{array}{l}\text { Pcpn. } \\
\text { Potential (\%) }\end{array}$ & 0 & 0 & 0 & 0 & 0 & 0 & 0 & 5 & 5 & 5 & 5 & 5 & 5 & 5 & 5 & 5 & 5 & 5 & 5 & 5 & 5 & 5 & 5 & 5 \\
\hline $\begin{array}{l}\text { Rel. Humidity } \\
\text { (\%) }\end{array}$ & 56 & 62 & 64 & 69 & 73 & 75 & 76 & 77 & 72 & 63 & 56 & 48 & 41 & 36 & 34 & 31 & 30 & 30 & 32 & 33 & 38 & 45 & 50 & 55 \\
\hline Thunder & - & - & -- & - & - & - & - & - & - & - & - & - & - & - & - & - & - & - & - & - & - & - & - & - \\
\hline Rain & - & - & - & - & - & - & - & - & - & - & - & - & - & - & - & - & - & - & - & - & - & - & - & - \\
\hline
\end{tabular}

Fig. 2.2: Hourly NOAA forecast, Aug. $31^{\text {st }} 2013$.

\subsubsection{Weather Variables}

The available weather variables of interest for this dissertation were temperature, relative humidity, dew point, sky coverage, visibility, wind speed and direction. These weather variables can be categorized into continuous or discrete variables. Of the two categories, the former one includes temperature, relative humidity, dew point, and wind speed. The latter includes sky coverage, visibility, and wind direction. Continuous variables are better suited for the proposed NB model because discrete variables represent some finite states, which cannot be shown in the process of classification of various values 
between two states. Thus, the three weather variables, temperature, relative humidity, and dew point, are used as features in the NB model and the sky coverage, which represents the different types of clouds is used to figure out the impact on GHI variation before the NB classification is performed. Also, the study in [43] supports the use of the three continuous weather variables that indicate the certain correlation with GHI. With this point of view [43], the properties of the three variables are summarized as follows.

\section{1) Temperature}

The hourly measured temperature values show a similar trend as with GHI. Given the data set, temperature varies in the range of $23 \mathrm{~F}$ and $112 \mathrm{~F}$.

2) Relative humidity

Relative humidity is the ratio of the density of water vapor to the saturated water vapor in the air. Relative humidity shows opposite trend with GHI. It shows typical ranges between $10 \%$ and $99 \%$.

3) Dew point

Dew point is the temperature at which the relative humidity reaches $100 \%$. Dew point varies from $1 \mathrm{~F}$ to $77 \mathrm{~F}$ given the data set.

\subsubsection{Solar Irradiance}

GHI measurements can be an important variable to produce and forecast PV power output. Since the values of GHI are proportional to the PV power curve, GHI is appropriate for forecasting PV power output. 
Fig. 2.3 shows hourly actual GHI of which the maximum value is about 1000 $\mathrm{Wh} / \mathrm{m}^{2}$ in August. In order to classify GHI in the proposed NB model, the measured range of GHI values is divided into several groups, so that the classified GHI according to weather features belongs to certain finite groups.

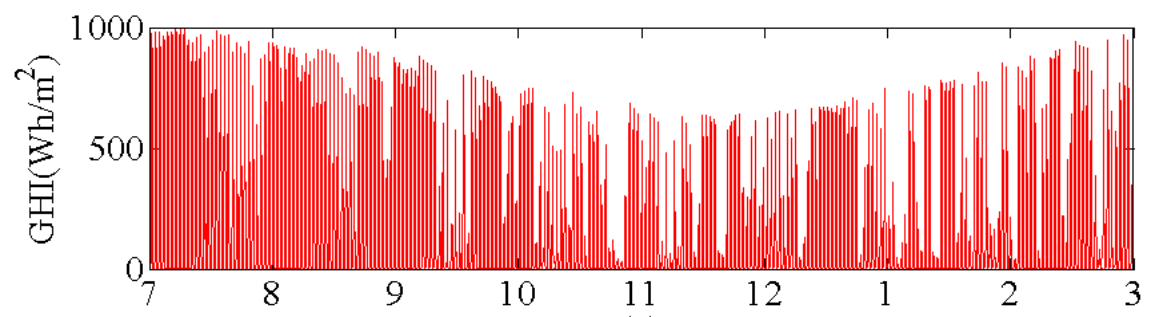

(a)

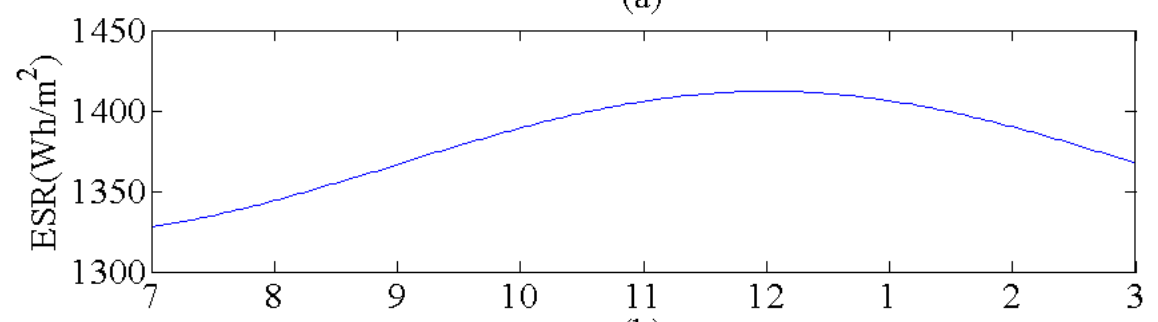

(b)

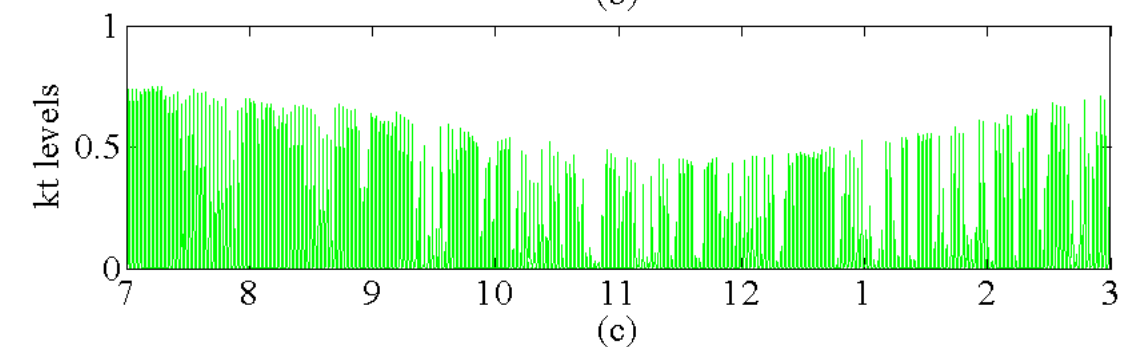

August through March, 2013-2014

Fig. 2.3: 8 months solar irradiance data set used in NB model: (a) hourly actual GHI, (b) normalization factor ESR, and (c) clear index $k t$.

Then, GHI is transformed by the extraterrestrial solar radiation (ESR) called clear index $k t$ in [44] since ESR has greater values than GHI. The calculation of $k t$ is done as, 


$$
\begin{gathered}
\left.\beta=\frac{2 \pi n}{365} \quad \text { [radians }\right] \\
\left(\frac{R_{a v}}{R}\right)^{2}=1.00011+0.034221 \cdot \cos (\beta)+0.00128 \cdot \sin (\beta) \\
+0.000719 \cdot \cos (2 \beta)+0.000077 \cdot \sin (2 \beta) \\
E S R=S C \cdot\left(\frac{R_{a v}}{R}\right)^{2}\left[\mathrm{~W} / \mathrm{m}^{2}\right] \\
k t=\frac{G H I}{E S R}
\end{gathered}
$$

where $S C$ is solar constant $\left(1367 \mathrm{~W} / \mathrm{m}^{2}\right)$ and $R_{a v}$ and $R$ are the mean sun-earth distance and the actual sun-earth distance, which vary depending on the day of year. ESR in Fig. 2.3 (b) has the range of 1320 to $1420 \mathrm{~W} / \mathrm{m}^{2}$, so the range of $k t$ is derived from 0 to 1. Fig. 2.3 (c) shows $k t$ levels that have the similar trend of GHI as in Fig. 2.3 (a). In the proposed NB model, $100 \mathrm{kt}$ levels are chosen based on a step size of 0.01 . After the NB model is performed, the classified $k t$ levels would be converted back to GHI values by multiplying $k t$ with ESR in (2.4). 


\subsection{Renewable Source Model}

GHI measurements are used for the PV power output calculation. PV modules exhibit a difference between the inside and outside temperature. Usually, the PV inside temperature is higher than the outside temperature. For simplicity of calculations, the assumption is that the temperature inside and outside the PV module is the same [45] and the power output by a PV module can be expressed as,

$$
P=G H I \times A \times \eta_{P V}
$$

where $\mathrm{A}$ is the PV module area $\left(\mathrm{m}^{2}\right)$ and $\eta_{P V}$ is the module conversion efficiency. With a USA MX60-240 PV module which has a size of $1.7 \mathrm{~m}^{2}$ in this study, assumes an efficiency of $14 \%$ and maximum power of $240 \mathrm{~W}$.

For the wind power output, a theoretical turbine wind speed-power output curve is used. The Excel $10 \mathrm{~kW}$ wind turbine, BergeyBWE10, is considered, which is comprised of 3-blade horizontal axis (7 $\mathrm{m}$ rotor diameter) with $5.6 \mathrm{mph}$ cut-in wind speed and maximum power of $10 \mathrm{~kW}$ at $26 \mathrm{mph}$.

Fig. 2.4 shows monthly renewable energy profiles, which are according to the given two configurations in a cellular network that include 6 PV modules and a wind turbine (blue) and 20 PV panels (red) from April 2013 to March 2014. As shown in Fig. 2.4, the renewable sources tend to be affected by seasonality. Solar energy shows the higher harvest in summer, but the wind energy shows more generation in winter. 


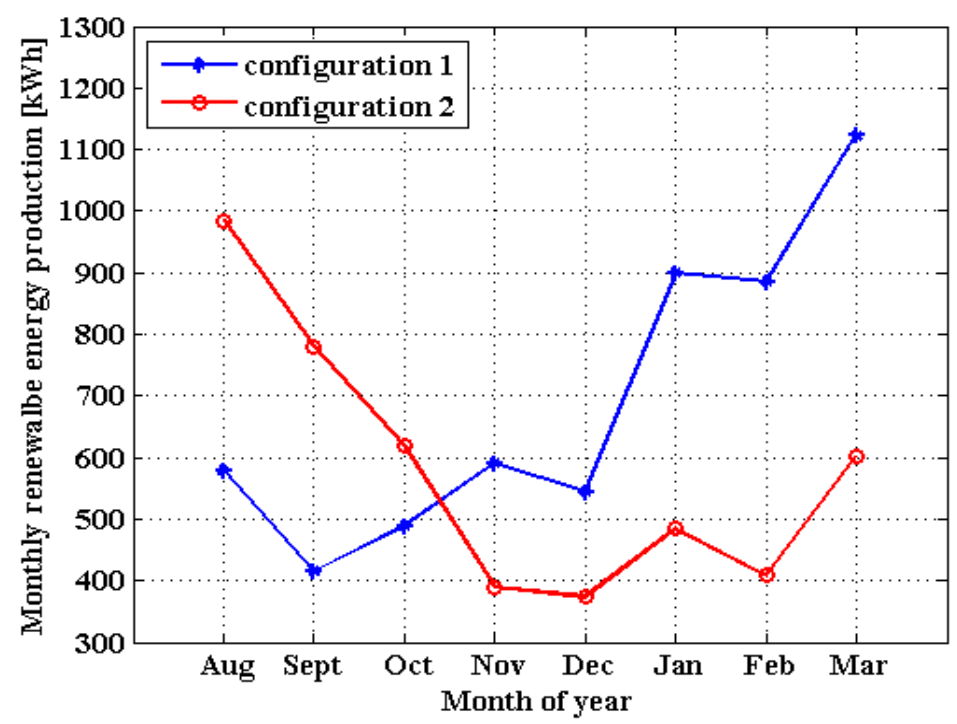

Fig. 2.4: Monthly average energy production in two configurations from August 2013 to March 2014.

\subsection{BS MODEL}

Fig. 2.5 represents a generalized scheme of a BS which is comprised of a baseband (BB) unit and multiple transceivers that consist of radio frequency (RF) part, power amplifier (PA), and antennas. With a $48 \mathrm{~V}$ input voltage, the BS contains a DC-DC converter, cooling system, and an AC-DC converter for a connection to the power gird. In traditional deployments, BS equipment is located far from the antenna which causes high power losses by long feeder cables. However, in a LTE BS, the feeder cable losses can be reduced with a remote radio unit (RRU) layout, which allows the equipment (RF and PA) to be close to the antenna [6]. 


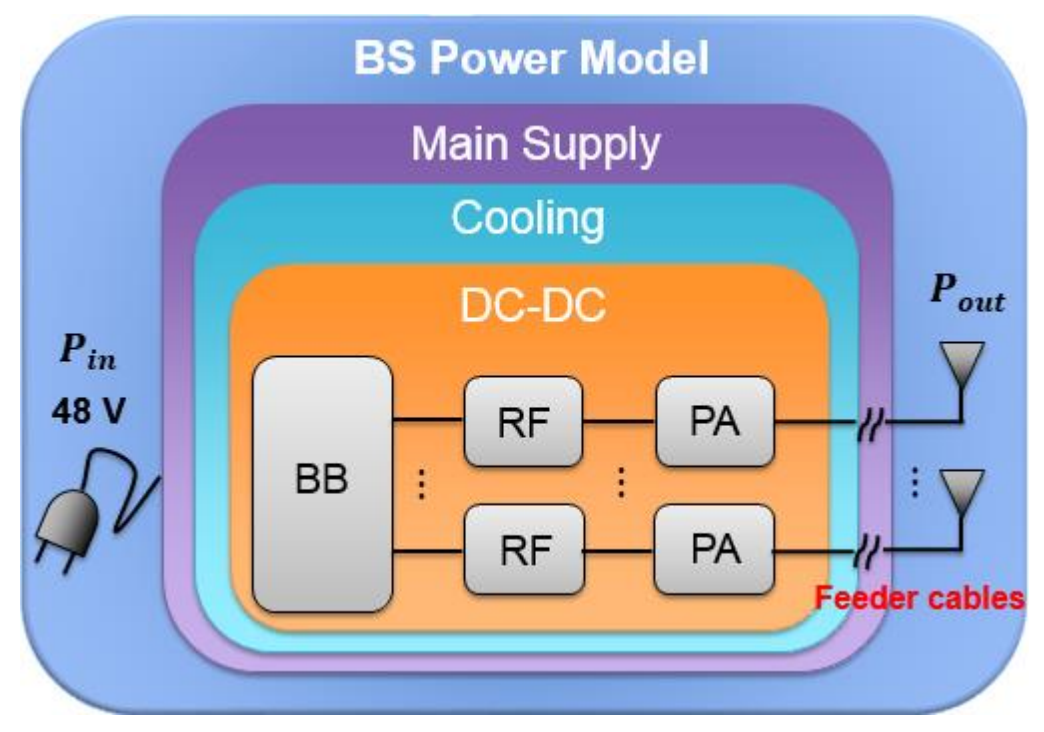

Fig. 2.5: Simplified scheme of a BS

Since LTE networks adopt an orthogonal frequency division multiplexing (OFDM) modulation scheme, which allows PA to operate in a linear region between 6 and $12 \mathrm{~dB}$, the most power is consumed by PA part in a LTE BS.

The peak power consumption of a LTE macro BS is about $1350 \mathrm{~W}$ with the 10 MHz bandwidth, 3 sectors, and 2 antennas per sector. However, when the RRU layout is considered, the peak power consumption deceases to about $800 \mathrm{~W}$ due to power efficiency by cooling system and PA part. At the peak power consumption (full load), the most power is consumed by the PA part, which accounts for about $60 \%$ of the total power consumption in the macro BSs. The actual power consumption in a BS depends on PA power consumption, which in turn represents a linear relation with the traffic carried by the BS as shown in Fig. 2.6 and (2.6) 


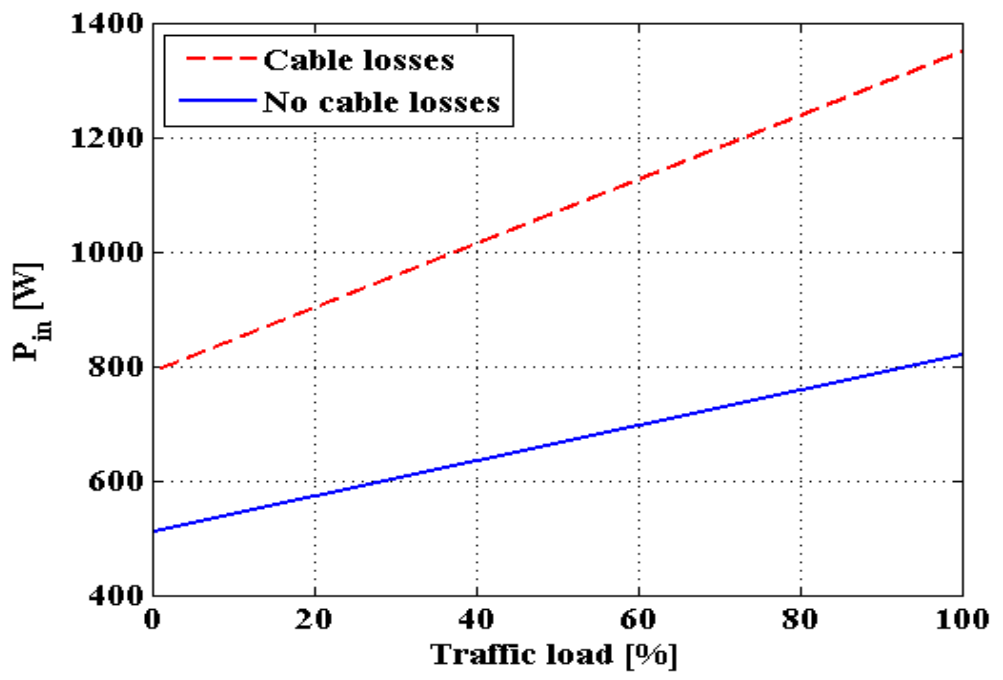

Fig. 2.6: BS power consumption vs traffic load

$$
P_{\text {in }}=\left\{\begin{array}{cc}
N_{T R X} \cdot\left(P_{0}+\Delta_{p} P_{\text {out }}\right), & 0<P_{\text {out }} \leq P_{\text {max }} \\
N_{\text {TRX }} \cdot P_{\text {sleep }}, & P_{\text {out }}=0
\end{array}\right.
$$

where $N_{T R X}$ is the number of antennas, $P_{0}$ is the linear model parameter when the RF output is zero, $P_{\max }$ is the maximum PA power at full load, $\Delta_{p}$ is the slope of relation between power consumption and load, and $P_{\text {sleep }}$ is the power output value when the BS is in sleep mode [6].

The proposed cellular network is considered in an industrial or residential urban or suburban area where a BS in each site has a coverage radius of 250 meters. Based on a macro BS, transmitting 3 sectors with $2 \times 2$ MIMO (two transmitters and two receivers) antenna configuration and transmitting power of $40 \mathrm{dBm}$ are assumed. Also, frequency 
reuse factor of 3 and $10 \mathrm{MHz}$ system bandwidth are assumed as in [46]. From the hourly average traffic profiles as shown in Fig. 2.7 [47], a BS's power consumption is calculated using the model from [48], which can shape the traffic corresponding to the forecasted renewable energy and battery SOC. Based on (2.6) the power consumption is expressed by

$$
B S_{t}=N_{T R} \cdot\left(97 \cdot \sigma_{t} \cdot T P_{t}+65\right)
$$

where $N_{T R}$ is 6 (number of antennas per sector, 2, times the number of sectors per BS, 3) when the BS operates and $\sigma_{t}$ is traffic shaping factor (control factor) ranging from 0.3 (maximum traffic shaping) to 1 (no traffic shaping) that affects the dynamic behavior of the BS power demand. $T P_{t}$ and the number of 97 and 65 are traffic profile in $t$ th trial (hour) in Fig. 2.7 and constant power terms which are determined in [48]. More specifically, the power consumption term in (2.7) can be divided into two components: a constant and a dynamic component. The constant term of $65 \mathrm{~W}$ includes the basic power consumption in a BS such as baseband interface, cooling, and radio resource overhead (pilot signals), etc. The dynamic component, which has the approximately linear relationship with the utilization of resource blocks, depends on the cellular traffic $T P_{t}$. 


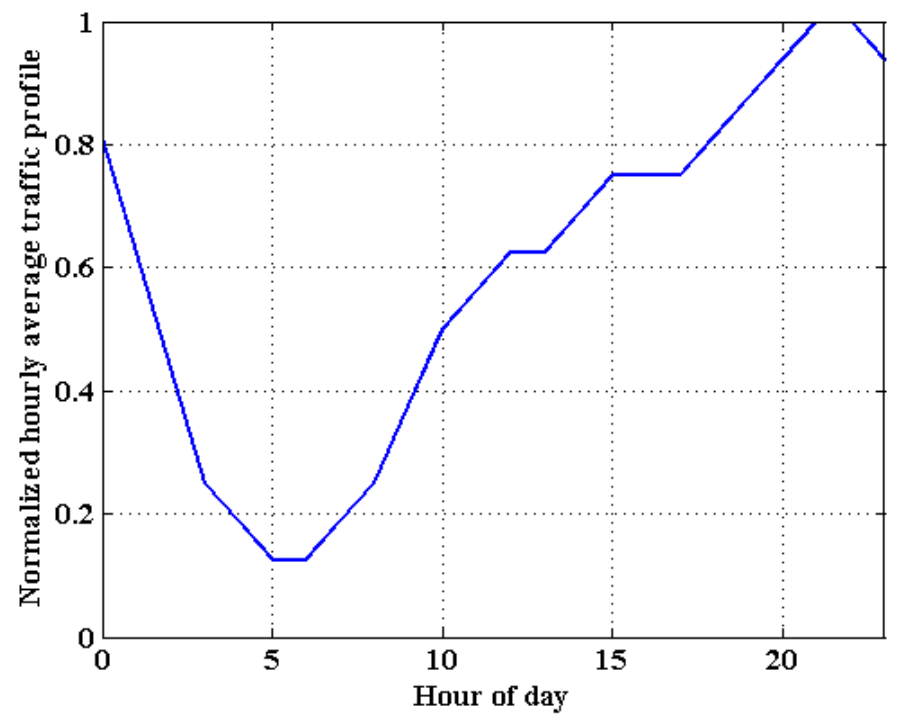

Fig. 2.7: Normalized hourly average traffic profile in Europe [47]. 


\section{Chapter 3. Renewable Energy Prediction Model}

\subsection{LiterATURE REVIEW}

Present state and federal agencies are in the process of increasing the penetration of PV systems on local utility grids. PV power is desirable for its minimal environmental impact, reduced reliance on oil and improved secure electricity supply [49]. Moreover, since the efficiency of PV cells is expected to improve by 44 to 50 percent in the future [50], the contribution of power production by PV systems to electricity grids will increase significantly. However, as the grid-connected PV systems increase, grid operators and system planners will also be more concerned about the PV system's power output fluctuation. Since the power output varies significantly depending on the solar irradiance variability, some potential risks on the utility grid or system would be expected such as scheduling the primary and spinning reserve capacity and the control of the voltage levels. As a back up plan, an energy storage can assist in supplying more sustainable power against the solar irradiance variability [51], but in connection with power dispatching plan, transmission scheduling, and a-day-ahead market industry still needs the accurate solar irradiance forecast techniques based on an hourly resolution in order to reduce the uncertainty of the variability [52]. Furthermore, the forecasting solar irradiance a day ahead or even more can be helpful in agriculture by constructing temperature maps that are directly affected by the solar irradiance [53].

Until recently, many studies have worked on forecasting GHI or solar power output directly. The solar forecast techniques can be divided into two classes by data resolution. 
For the high resolution (i.e., less than one hour), most time series techniques such as regression, autoregressive integrated moving average (ARIMA) [54], artificial neural networks (ANN), and hybrid models that are combination of regressions with ANN all show good accuracy compared to the reference model in [55]. For the a-day-ahead forecast with hourly resolution, the study in [56] forecasts solar irradiance using ANN with multilayer perceptron (MLP) model during sunny and cloudy days. For the sunny days, the forecast accuracy is verified with correlation coefficient of 98.95 to $99.96 \%$, relative mean bias error (RMBE) of -6.43 to $32 \%$, and relative root mean square error (RRMSE) of 18.98 to $67.08 \%$. Similar to [56], the study in [43] proposes solar power forecast using ANN in several weather types. For sunny days, the correlation coefficient shows 98.43 to $99.39 \%$, and mean absolute percentage error (MAPE) represents 8.29 to $10.8 \%$. However, over the several hours or on the cloudy or rainy days, the majority of them show larger errors due to the severe transitions in the solar irradiance and weather change by a diurnal cycle. The studies in [57] and [58] also have developed solar irradiance forecast hour-by-hour or dayby-day using ANN. However, they produced results with large errors given the previous poorly forecasted value.

The ANN forecast techniques are frequently used in many areas and works well these days, but it also has drawbacks. Complicated architecture, large training data set, and choosing the optimal number of hidden layers and input nodes for the better results still remain as issues. Furthermore, the use of different test criteria (i.e., comparison to the unclear reference model, normalization factor or test duration) or performance testing under the several defined weather types (i.e., sunny, foggy, rainy, and cloudy day) makes 
it difficult to compare with other developed models and understand with real-time weather, which can be a series of changes without certain weather types for a day or few days. Although these forecast models can be a baseline against which certain change occurs in its similar environment or evaluated according with its weather types, it is hard to compare generally with other developed values or evaluate for the whole period of the test, which is close to real-life situations.

In order to solve these problems, this paper proposes a simple probabilistic classification method, NB classifier for two-days-ahead GHI forecast. Since the variability of solar irradiance raises many problems as mentioned above, the proposed NB model considers two main characteristics of solar irradiance (diurnal cycle and daytime variability) in order to improve the forecast accuracy by avoiding the overlapping classification. Based on the Bayes' theorem, conditional probabilities are calculated which not only include the prior knowledge of solar irradiance but also account for uncertainties in the future solar irradiance variability. Using the four forecasted and observed weather variables such as temperature, relative humidity, dew point, and sky coverage, two day ahead solar irradiance is predicted with hourly resolution. Moreover, the proposed NB model, which consider above two characteristics, can provide hourly GHI forecast as well as a daily total GHI without the effect of the previous forecasted results.

The two-day-ahead forecast accuracy is validated with the statistical values and several error criteria. For the whole data ( 8 months), the proposed NB model indicates RMBE of $2.73 \%$, and RRMSE of $41.7 \%$ based on the mean GHI of $333.04 \mathrm{Wh} / \mathrm{m}^{2}$. Especially, August, which represents mostly clear days, satisfies the guideline for the 
evaluation of two days ahead forecast, in which the acceptable limit of RRMSE is about $23 \%$ in [52]. Moreover, the mixed weather types for 4 days results are evaluated with error criteria in order to compare the existing weather type models.

\subsection{Solar Power Prediction Model}

In this section, the proposed NB model is constructed as consisting of three steps. In the first step, the daytime hours in the NB model are partitioned into subsets in order to avoid an overlapping classification. In the second step, the observed weather variables are filtered by the five states of forecasted sky coverage to improve the classification accuracy. In the final step, the proposed NB model is performed based on a Gaussian kernel estimation and with the forecasted weather values as input.

\subsubsection{Step 1: Deterministic Characteristic of GHI}

In many studies, GHI is considered as a random variable due to its uncertainty, which arises from the random weather changes. As shown in Fig. 3.1, on a clear day, the values of GHI over time follow what can be best described as a bell-shaped curve, increasing until noon and decreasing thereafter until sunset. This trend allows the random component in the GHI can be estimated by leveraging the observations that the deterministic characteristic (24 hour cycle) in a day dominates the overall GHI value. However, this trend also causes the overlapping classification because there are two overlapped points over the GHI values (red points in Fig. 3.1), when the whole day (24 
hours) data are applied in the proposed NB model. And this trend is also observed on an overcast day. Therefore, the basic idea for step 1 is to reduce the GHI variation as well as the overlapping classification by limiting the classification range to one hour.

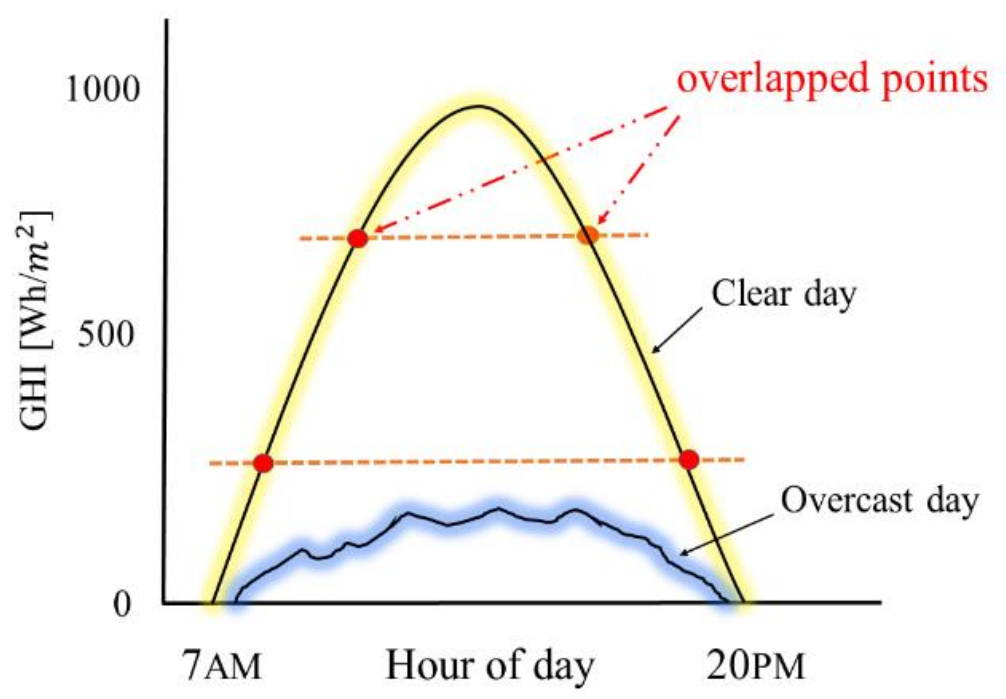

Fig. 3.1: Deterministic characteristic of GHI on a clear day and an overcast day.

Fig. 3.2 shows that, after excluding the trivial hours with no sunlight, a day can be partitioned into 14 hour-long subsets (i.e., daytime hours, 7:00 A.M. to 20:00 P.M.). In this paper, we assume that the daylight hour is fourteen hours, but it can be adjusted depending on location and season (i.e., latitude or longitude and summer or winter). The rows of the matrix $o b s_{\text {whole day }}$ correspond to each day in the training data (here 30 days) and the columns correspond to each daylight hour. The elements in the matrix, $W_{o b s, h}=$ $\left[\begin{array}{llll}V_{T e m p, h} & V_{R H, h} & V_{D P, h} & V_{S C, h}\end{array}\right] \in \mathbb{R}^{1 \times 4}$, represent the feature vectors consisting of the 
temperature, relative humidity, dew point, and sky coverage observation values, respectively. The partitioning of the daily data set into hours increases the number of iterations the NB classifier requires to process the data. However, the speed of calculation is not impacted due to the simplicity of the NB classifier.

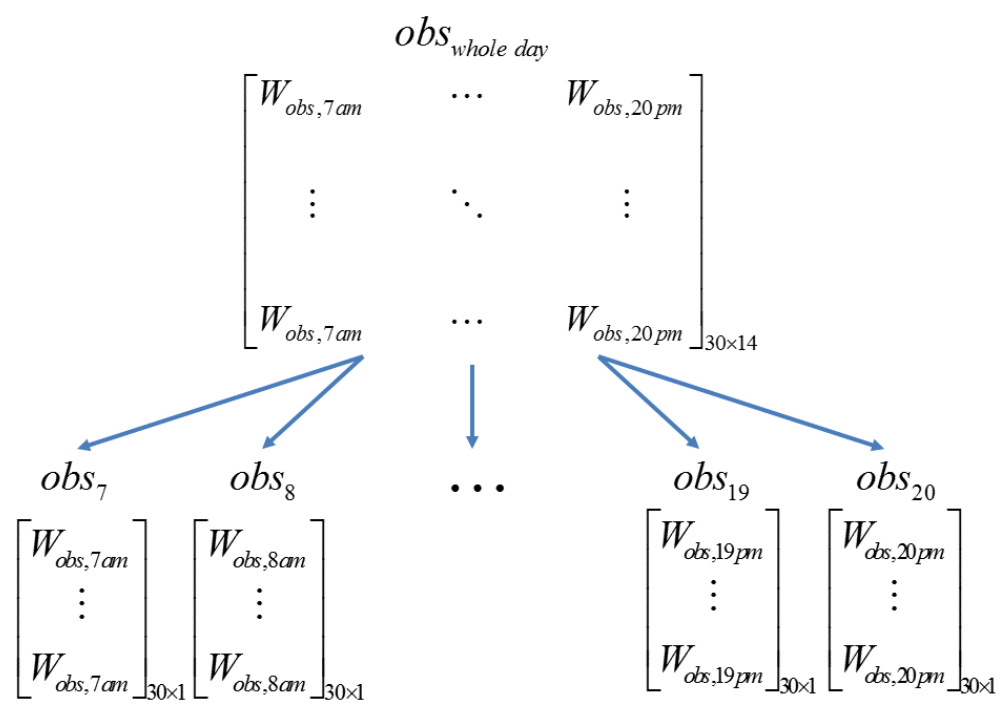

Fig. 3.2: The fourteen one-hour observation training sets obtained from separation of the daylight data (step 1).

\subsubsection{Step 2: GHI Variation by Clouds}

Sky coverage plays a pivotal role in the GHI forecast model. Other factors may also affect GHI, e.g. dust, trees, buildings etc., but most disturbances occur due to cloud movement. In contrast to the other observed variables, sky coverage is a discrete quantity. The sky coverage can be divided into 5 states according to the reported weather data 
observations [33]: clear (0-1\%), mostly clear (2-23\%), partly cloudy (24-48\%), mostly cloudy (49-81\%), and overcast sky (82-100\%). Fig. 3.3 represents how the new training sets are obtained. The 14 observations from a single day that make up the training sets from step 1, are filtered individually by following these 5 states of the forecasted sky coverage.

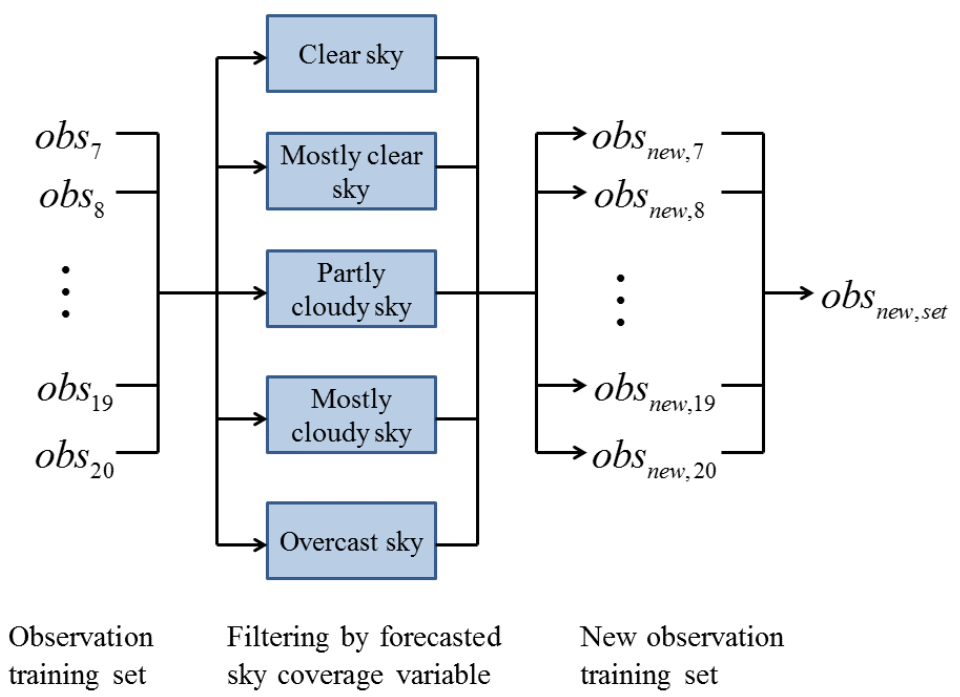

Fig. 3.3: Filtered training set using the forecasted sky coverage variable (step 2).

For a better understanding of step 2, an example can be given as follows. If the next day sky coverage state at 8 A.M. indicates clear sky and 4 days training data are assumed as in (3.1), the same states (clear sky) of the feature vectors $W_{o b s, 8}$ in the matrix $o b s_{8}$ are selected and the rest of feature vectors including the other states (i.e., overcast and partly cloudy) are excluded in the $o b s_{8}$. Therefore, the new observation training set matrix, $o b s_{n e w, 8}$ is determined as in (3.2). In step 2, this process is repeated continuously for the entire observation training set $\left(o b s_{8}\right.$ to $\left.o b s_{19}\right)$ in order to improve the estimate of 
the feature vector (weather variables) given the GHI observations. This process will be discussed further in the next section while discussing the NB classifier.

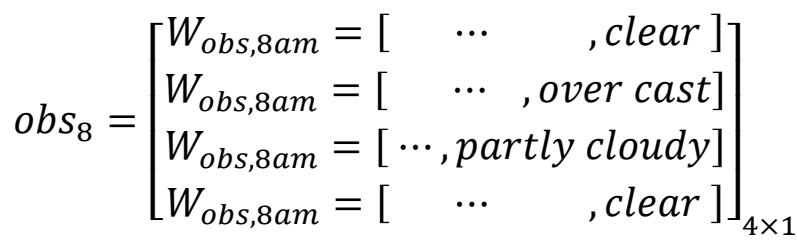

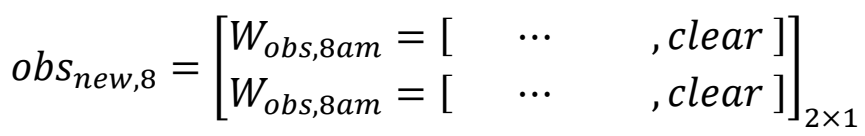

\subsubsection{Step 3: NB Classifier}

The NB classifier is an effective probabilistic classification algorithm. Based on Bayes' theorem, classification is performed with the assumption that the features (the weather variables) are independent of each other given the corresponding classes (the levels or values of the $k t$ ). This assumption considerably simplifies the training step of the proposed algorithm for forecasting, and for that reason the calculations are fast while the performance is highly accurate in many practical applications [59].

Fig. 3.4 represents the process of the NB model. The input value, in conjunction with the observation training set, draws the output value $k t_{N B} \in\{1, \cdots, 100\}$ that belong to each hour in the daytime. Unlike the observation feature $W_{o b s, h} \in \mathbb{R}^{1 \times 4}$ in step 1 , the forecasted feature vector $W_{f c s t, h} \in \mathbb{R}^{1 \times 3}$, which includes $V_{T e m p, h}, V_{R H, h}$, and $V_{D P, h}$ is 
used as an input for the proposed NB model. The relationship between the input and output in Fig. 3.4 can be expressed as follows:

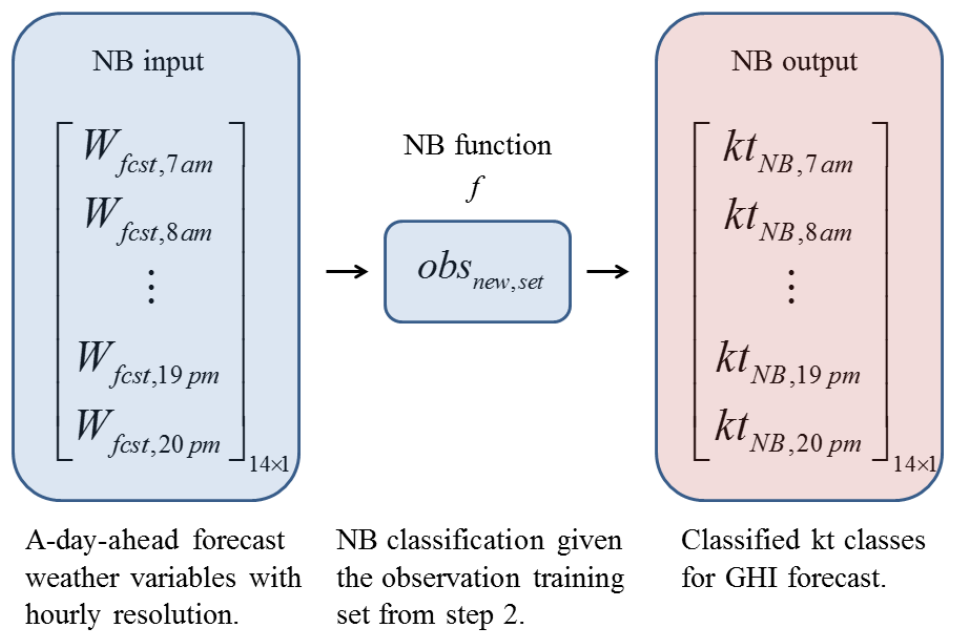

Fig. 3.4: Modified NB classification using forecasted weather variables (step 3).

$$
k t(h+48)=f\left(V_{T e m p}(h+48), V_{R H}(h+48), V_{D P}(h+48)\right)
$$

where the function $f$ represents the NB classification.

The fundamental idea of Bayes' theorem is that the probability of class $C \in$ $\{1, \cdots, l\}$, where $l$ is integer including the class levels, in other words the set of values that GHI can take (here, $l=100$ ) can be estimated based on the observations features. For ease of notation, hereafter let $\left[\mathrm{V}_{1}, \mathrm{~V}_{2}, V_{3}\right]=\left[\begin{array}{lll}V_{T e m p} & V_{R H} & V_{D P}\end{array}\right]$ and $V_{i}$ represents the features $V_{1}, V_{2}, V_{3}$ for $i=1,2,3$. With multiple features, Bayes' theorem is expressed as, 


$$
P\left(C \mid V_{1}, V_{2}, V_{3}\right)=\frac{P\left(V_{1}, V_{2}, V_{3} \mid C\right) \cdot P(C)}{P\left(V_{1}, V_{2}, V_{3}\right)} .
$$

The prior probability of $C, P(C)$, is obtained for a given data set before the features are observed, but the posterior probability $P\left(C \mid V_{1}, V_{2}, V_{3}\right)$ is obtained after the features are observed. With the independence assumption, the multiple conditional probabilities of (3.4) can be described as

$$
P\left(C \mid V_{1}, V_{2}, V_{3}\right)=\frac{P\left(V_{1} \mid C\right) \cdot P\left(V_{2} \mid C\right) \cdot P\left(V_{3} \mid C\right) \cdot P(C)}{P\left(V_{1}, V_{2}, V_{3}\right)} .
$$

Equation (3.5) represents the effect of each feature $V_{i}$ on a given class $C$ that is independent of the other features. Moreover, the denominator in (3.5) can be omitted in the calculation since $P\left(V_{1}, V_{2}, V_{3}\right)$ has the same effect on all the classes.

There are two parts in obtaining the posterior estimate $P\left(C \mid V_{1}, V_{2}, V_{3}\right)$. First part is estimating the conditionals $P\left(V_{i} \mid C\right)$ using kernel density function as explained next. The second part is using the estimate of the conditional probabilities $P\left(V_{i} \mid C\right)$ in part 1 into (3.5) to obtain the posterior $P\left(C \mid V_{1}, V_{2}, V_{3}\right)$. 


\subsubsection{Estimation of $P\left(V_{i} \mid C\right)$ : Kernel methods}

The estimation of $P\left(V_{i} \mid C\right)$ requires itself an estimation of the probability density functions (PDF) for the random variables involved. This estimation can be implemented using parametric or non-parametric methods. The parametric estimation for the PDF assumes that it is a member of some parametric family of distributions, e.g. a normal distribution $N\left(\mu, \sigma^{2}\right)$. When the assumption is correct, the parameters (mean $\mu$ and standard deviation $\sigma$ in case of the Gaussian) can be easily estimated from the data. However, when the underlying distribution generating the data has multiple modes or a skewed shape, the probability calculation might be wrong, due to the restrictions imposed by the choice of the distributions family.

Non-parametric kernel density estimation can deal with large variations in the features. In other words, the kernel density estimation is not necessary for the assumption that the features follow some generic probability distributions as mentioned above. In addition, since the GHI classification is a nonlinear problem [45], the kernel density function may be preferable to the estimation of $P\left(V_{i} \mid C\right)$. Therefore, this paper uses kernel density function based on Gaussian kernel in order to estimate $P\left(V_{i} \mid C\right)$. Gaussian kernel for each feature $V_{i}$ as follow

$$
K_{g a u}\left(V_{i, n}, \sigma_{i}\right)=\exp \left(\frac{-V_{i, n}{ }^{2}}{2{\sigma_{i}}^{2}}\right)
$$


where $V_{i, n}$ indicates $n$ observations $\left(V_{i, 1}, V_{i, 2}, \cdots, V_{i, n}\right)$ and $\sigma_{i}$ represents bandwidth. The bandwidth has a decisive effect on the decay of the Gaussian kernel in (3.6). However, the methods used to choose $\sigma_{i}$ are seldom discussed in related works. The bandwidth $\sigma_{i}$ can be determined from an estimator $\hat{\sigma}_{i}$, which is a combination of the inter-quartile range $\hat{R}_{i}$ and a rule-of-thumb bandwidth [60]. First, the interquartile range is $\hat{R}_{i}$ is determined as

$$
\widehat{R}_{\iota}=V_{i, Q 3}-V_{i, Q 1}
$$

which indicates that the interquartile range $\hat{R}_{i}$, is the length of the interval in the support of the feature $V_{i}$ between the upper quartile of $75 \%\left(V_{i, Q 3}\right)$ and the lower quartile of $25 \%$ $\left(V_{i, Q 1}\right)$. Equation (3.7) also can be transformed into the standardized Z-scale [61], which has a Gaussian with mean of 0 and standard deviation of 1 as shown in (3.8) by rescaling the horizontal axis with the feature mean $E\left(V_{i}\right)$ and standard deviation $\sigma\left(V_{i}\right)$.

$$
Z_{i}=\frac{V_{i}-E\left(V_{i}\right)}{\sigma\left(V_{i}\right)}
$$

Based on (3.8), then, the inter-quartile range $\hat{R}_{i}$ in (3.7) can be derived as

$$
\hat{R}_{i}=\left(E\left(V_{i}\right)+\sigma\left(V_{i}\right) Z_{Q 3}\right)-\left(E\left(V_{i}\right)+\sigma\left(V_{i}\right) Z_{Q 1}\right)
$$




$$
\begin{gathered}
=\sigma\left(V_{i}\right)(0.675-(-0.675))=1.35 \sigma\left(V_{i}\right) \\
\sigma\left(V_{i}\right)=\frac{\hat{R}_{i}}{1.35} .
\end{gathered}
$$

The standard deviation $\sigma\left(V_{i}\right)$ in (3.10) is then plugged into the rule-of-thumb bandwidth $\hat{\sigma}_{i, r o t}$ in (3.11), where $n$ represents the number of hourly data in feature $V_{i}$.

$$
\begin{gathered}
\hat{\sigma}_{i, r o t}=\left(\frac{4 \sigma\left(V_{i}\right)^{5}}{3 n}\right)^{0.2} \approx 1.06 \sigma\left(V_{i}\right) n^{-0.2} \\
\hat{\sigma}_{i, r o t}=1.06 \frac{\hat{R}_{i}}{1.35} n^{-0.2}
\end{gathered}
$$

\subsubsection{Update Posterior of $C$ : Calculating the value of $P\left(C \mid V_{i}\right)$}

Once the bandwidth estimator $\hat{\sigma}_{i, r o t}$ in (3.12) is derived, the above process, (3.6) through (3.12), is repeated in the training step in order to estimate a posterior density of each feature in each class using the Gaussian kernel density estimation. Based on the training step, the naïve Bayes classifier can be expressed as,

$$
k t_{N B}=\underset{C \in\{1, \cdots, l\}}{\arg \max } P(C) \prod_{i} P\left(V_{i} \mid C\right)
$$


where $k t_{N B}$ stands for the target class value, chosen to be the one maximizing the probability (3.13). Therefore, this proposed NB model is used to estimate the hourly GHI by multiplying ESR in (2.4) with $k t_{N B}$ from (3.13). It is worth noticing that the $k t_{N B}$ value has interval $[(l-1) \times 0.01 ; l \times 0.01]$, where $l=1, \cdots, 100$ so the median value of 0.005 is used for the $k t_{N B}$ when the GHI is re-estimated. Furthermore, after training two-day-ahead average daily solar energy can be forecasted by summing the daily $k t_{N B, h}$ values regardless of the previous forecast results since the input values are obtained from the weather reports.

\subsection{TeSTING, ReSUlts, AND Discussion}

The outputs of the proposed NB model, $k t$ classes, are re-converted to GHI values to compare with actual GHI. In order to consider seasonal effects, several error tests are compared month by month from August 2013 to March 2014.

\subsubsection{Diagnostic Checking}

To comprehensively evaluate the forecasting performance clearly, multiple error metrics were calculated. These metrics were the mean bias error (MBE), mean absolute error (MAE), root mean square error (RMSE), relative mean bias error (RMBE), mean absolute percentage error (MAPE), and relative root mean square (RRMSE) and were evaluated as, 


$$
\begin{gathered}
E=G H I_{f c s t, i}-G H I_{o b s, i} \\
M B E=\frac{1}{N} \sum_{i=1}^{N}(E) \\
M A E=\frac{1}{N} \sum_{i=1}^{N}|E| \\
R M S E=\sqrt{\frac{1}{N} \sum_{i=1}^{N}(E)^{2}}
\end{gathered}
$$

$$
\begin{aligned}
& R M B E=\frac{M B E}{\frac{1}{N} \sum_{i=1}^{N} G H I_{o b s, i}} 100 \\
& M A P E=\frac{1}{N} \sum_{i=1}^{N} \frac{|E|}{G H I_{o b s, i}} 100
\end{aligned}
$$

$$
R R M S E=\frac{R M S E}{\frac{1}{N} \sum_{i=1}^{N} G H I_{o b s, i}} 100
$$


where subscript $i$ represents the $i$ th forecast and observation pair given the forecasting horizon length. For example, $N$ is equal to 434 (14 daily points $\times 31$ days) for August. With hourly resolution, the error evaluations are restricted to the daytime hours (14 points).

Depending on the various purposes, the error criteria can be mutually complementary for analyzing the forecast quality. Usually RMBE in (3.18), MAPE in (3.19), or RRMSE in (3.20) are used for forecast testing, but these are often unclear on the normalizing factor (measured mean or maximum value).

The MBE in (3.15) measures the tendency of the solar energy that is over-estimated or under-estimated given the forecasting period. For example, MBE can be directly used for an evaluation of real application such as PV energy storage system. The MAE in (3.16) measures an absolute difference that is less biased than the RMSE in (3.17) in large error cases. Similar to the MSE, the MAPE in (3.19) is widely used for forecasting performance tests, but the MAPE is not bounded in case that the errors are greater than actual value [62]. The RMSE represents the variation between forecasted and actual data that is usually used for short-term forecast (up to 48 hours ahead). RMBE in (3.18) and RRMSE in (3.20) show the relative MBE and RMSE values, which are normalized by the average of GHI within the observation period.

\subsubsection{Modeling Testing}

Before the forecasting results are discussed, it is important to note that summers in Austin show more clear days than the other seasons. Moreover, in order to avoid an infinite 
result during the computation procedure the denominators in (3.18), (3.19), and (3.20) are replaced by 1 during the nighttime, when the GHI result is close to zero.

\section{1) Monthly results: Seasonal effect}

Table 3.1 shows a summary of the statistical values relevant to the two day ahead GHI forecast. For the actual GHI the minimum, maximum, mean, and correlation coefficient $r$ are evaluated month by month with hourly resolution. From Table 3.1, August indicates the maximum GHI and standard deviation with the largest mean of 551.28 $\mathrm{Wh} / \mathrm{m}^{2}$ while December shows the smallest GHI and standard deviation with the smallest mean of $210.87 \mathrm{Wh} / \mathrm{m}^{2}$. Similar to GHI trend, $r$, which represents the linear relationship between actual and forecast value, also shows the maximum of $90.38 \%$ for August and minimum of $78.37 \%$ for December). In addition, the RRMSE shows an inverse proportionality with the GHI mean value (the minimum of $23 \%$ for August and the maximum $60.01 \%$ for December). The RMBE of August through November indicates an overestimation in the NB model while the rest of the months show the reverse tendency. More importantly, MBE and RMBE indicate $9.09 \mathrm{Wh} / \mathrm{m}^{2}$ and $2.73 \%$ for the 8 months test that show the better results that other metric. As shown in (3.15) and (3.18), the MBE and RMBE show the characteristic of a short-term prediction evaluation (not point prediction test) so predicting the power output of renewable sources, which are coupled with an energy storage, might be explained with the MBE and RMBE metric because the point prediction error during a given prediction period can be complemented by the stored energy in energy storage system. Particularly, the MAPE is not evaluated in the monthly analysis 
(Table 3.1) because of its unbounded property. This property will be discussed in more detail in the next sub-section.

Fig. 3.5 represents the relationship between actual and forecasted GHI for an eight months period. Fig. 3.5 (a) shows the scatter plot where a large correlation between actual and forecasted GHI can be visually seen through the linear regression shown by the results. Indeed, the correlation coefficient was measured equal to $86.33 \%$. In details, the results in Fig. 3.5 (a) indicate overestimation when the actual GHI represents lower values (about less than $300 \mathrm{Wh} / \mathrm{m}^{2}$ ). However, as the actual GHI increases, the forecasted GHI tends to predict the actual GHI more accurately. The cumulative distribution functions (CDF) depicted in Fig. 3.5 (b) also show that the actual GHI (blue) closely matches the forecasted GHI (red) when the actual GHI is greater than $300 \mathrm{Wh} / \mathrm{m}^{2}$.

\begin{tabular}{|ccccccccccc|}
\hline Month & $\begin{array}{c}\text { Min./Max } \\
\left(\mathrm{Wh} / \mathbf{m}^{2}\right)\end{array}$ & $\begin{array}{c}\text { Mean } \\
\left(\mathrm{Wh} / \mathbf{m}^{2}\right)\end{array}$ & $\begin{array}{c}\text { Std. } \\
\left(\mathrm{Wh} / \mathbf{m}^{2}\right)\end{array}$ & $r$ & $\begin{array}{c}\text { Training } \\
\text { days }\end{array}$ & $\begin{array}{c}\text { MBE } \\
\left(\mathrm{Wh} / \mathbf{m}^{2}\right)\end{array}$ & $\begin{array}{c}\text { MAE } \\
\left(\mathrm{Wh} / \mathbf{m}^{2}\right)\end{array}$ & $\begin{array}{c}\text { RMSE } \\
\left(\mathrm{Wh} / \mathbf{m}^{2}\right)\end{array}$ & $\begin{array}{c}\text { RMBE } \\
(\%)\end{array}$ & $\begin{array}{c}\text { RRMSE } \\
(\%)\end{array}$ \\
\hline Aug. & $5 / 999$ & 551.28 & 306.65 & 90.38 & 26 & 33.42 & 62.31 & 126.8 & 6.06 & 23 \\
\hline Sep. & $0 / 926$ & 452.85 & 302.79 & 86.55 & 26 & 23.46 & 78.18 & 143.64 & 5.18 & 31.72 \\
\hline Oct. & $0 / 879$ & 348.18 & 284.98 & 84.7 & 28 & 12.56 & 84.48 & 143.66 & 3.61 & 41.26 \\
\hline Nov. & $0 / 746$ & 227.11 & 232.94 & 87.29 & 36 & 40.49 & 76.51 & 117.22 & 17.83 & 51.64 \\
\hline Dec. & $0 / 643$ & 210.87 & 212.16 & 78.37 & 40 & 4.95 & 73.77 & 126.49 & 2.35 & 60.01 \\
\hline Jan. & $0 / 745$ & 273.30 & 240.28 & 91.42 & 50 & -11.09 & 57.04 & 91.03 & -4.06 & 33.32 \\
\hline Feb. & $0 / 853$ & 254.80 & 269.35 & 80.98 & 24 & -12.89 & 105.17 & 147.91 & -5.06 & 58.06 \\
\hline Mar. & $0 / 972$ & 338.68 & 300.83 & 80.9 & 30 & -32.85 & 107.37 & 167.32 & -9.7 & 49.4 \\
\hline Total & $0 / 999$ & 333.04 & 292.47 & 86.33 & 30 & 9.09 & 80.39 & 138.85 & 2.73 & 41.7 \\
\hline
\end{tabular}

Table 3.1: Summary of statistical values for actual GHI and prediction errors. 


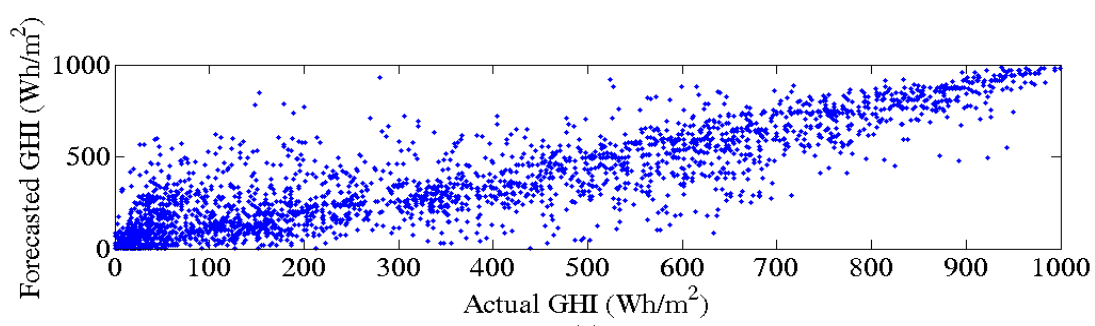

(a)

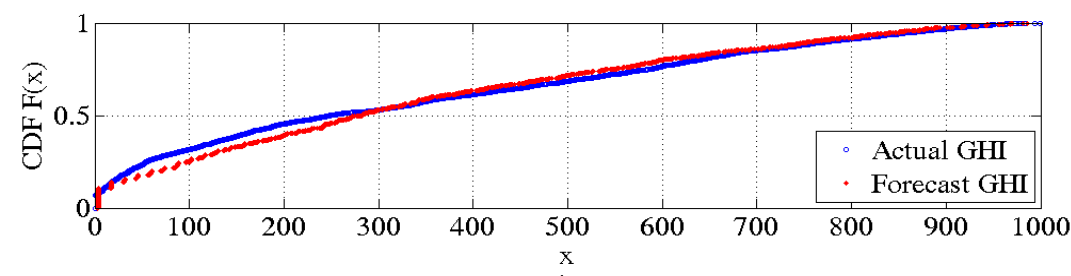

(b)

Fig. 3.5: Comparison between actual and forecasted GHI for 8 months:

(a) scatter plot and (b) cumulative function.

\section{2) 4 days results: Mixed weather vs specified weather types}

Fig. 3.6 to 3.9 compares the two-day-ahead forecast and actual GHI with the mixed weather types for 4 days. This mixed weather analysis is similar to the established analytical practices in [43], [56] in that test period is 4 days. However, the mixed weather analysis shows real-time weather change that is not specified with specific weather types (i.e., sunny, cloudy, or rainy days). On the days that are clear (August $5^{\text {th }}$ to $8^{\text {th }} 2013$ ), Fig. 3.6 and type 1 in Table 3.2 indicate that the forecasted GHI have a good agreement with the actual GHI. Fig. 3.7 shows a mix of 2 clear, 1 partly cloudy, and 1 overcast day (December $10^{\text {th }}$ to $13^{\text {rd }} 2013$ ) where the GHI decreases significantly at the last day. In contrast, the 3 overcast days and 1 clear day (February $24^{\text {th }}$ to $27^{\text {th }} 2014$ ) depicted in Fig. 3.8 show that GHI sharply increases on the last day. Although there are some differences between forecast and actual value on the last day in Fig. 3.7 and 3.8, the proposed NB 
model shows that the predicted GHI follows the actual values tendency in terms of MBE and RMBE metric. In addition, the following actual tendency can be proved with Table 3.2. For type 2 and 3 Table 3.2 indicate RRMSE of $50.94 \%$ and $29.45 \%$, but $r$ represents $84.16 \%$ and $96.86 \%$, which mean the predicted values significantly follows the actual GHI trend for 4 days. Lastly, Fig. 3.9 shows 1 clear and 3 partly cloudy days (March $25^{\text {th }}$ to $28^{\text {th }} 2014$ ), which represent the typical GHI variability by cloud movement. From the results (Fig. 3.6 to Fig. 3.9), most of differences between forecast and actual values occur in cloudy days. As shown in Fig. 3.7 and 3.8, there are significant differences in overcast days. However, since the magnitude of differences is relatively small compared to the cloudy days in Fig. 3.9, the RMBE and RRMSE in Table 3.2 represent lower values than the cloudy days (type 4). For the MAPE, Table 3.2 indicates a little different result. The MAPE shows a large error tendency on the cloudy or overcast days (type 2, 3 and 4) since the MAPE can have various values depending on the magnitude of the normalizing factor (actual GHI) as defined in (21). This nature of MAPE might not be explained from an analysis based on the similar consecutive specific weather type test that the predicting patterns can be trained under consistent weather condition than the mixed weather type analysis. 


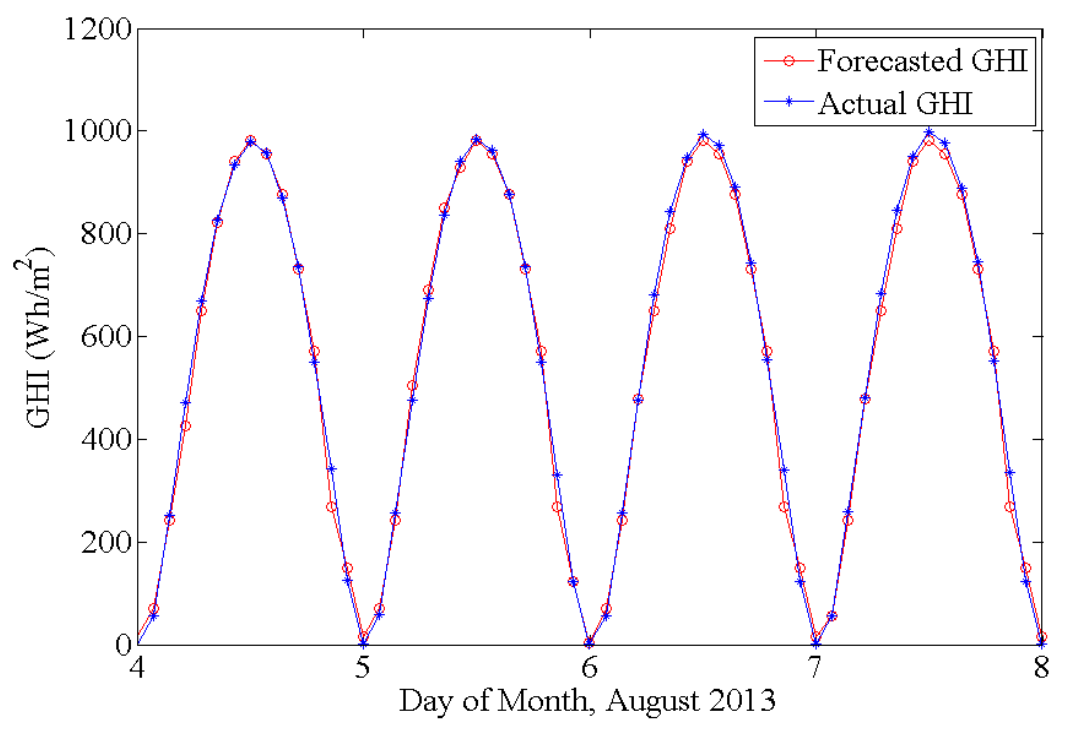

Fig. 3.6: Comparison between two days ahead predicted and actual GHI for clear 4 days (type 1 ).

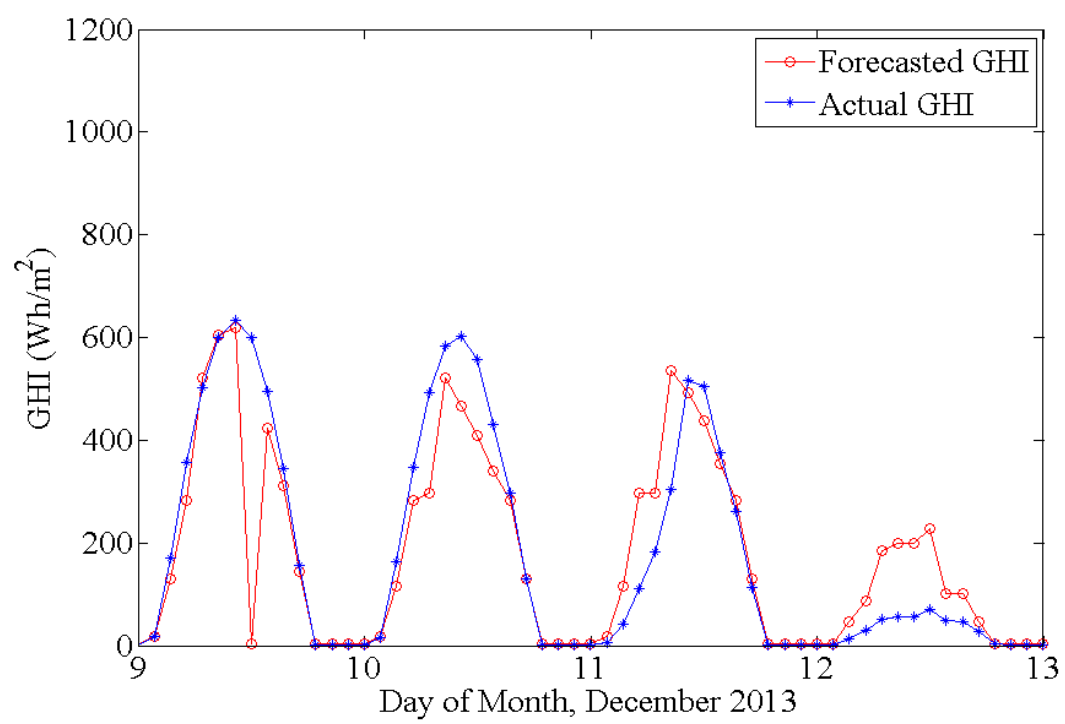

Fig. 3.7: Comparison between two days ahead predicted and actual GHI for 2 clear, 1 partly cloudy, and 1 overcast day (type 2). 


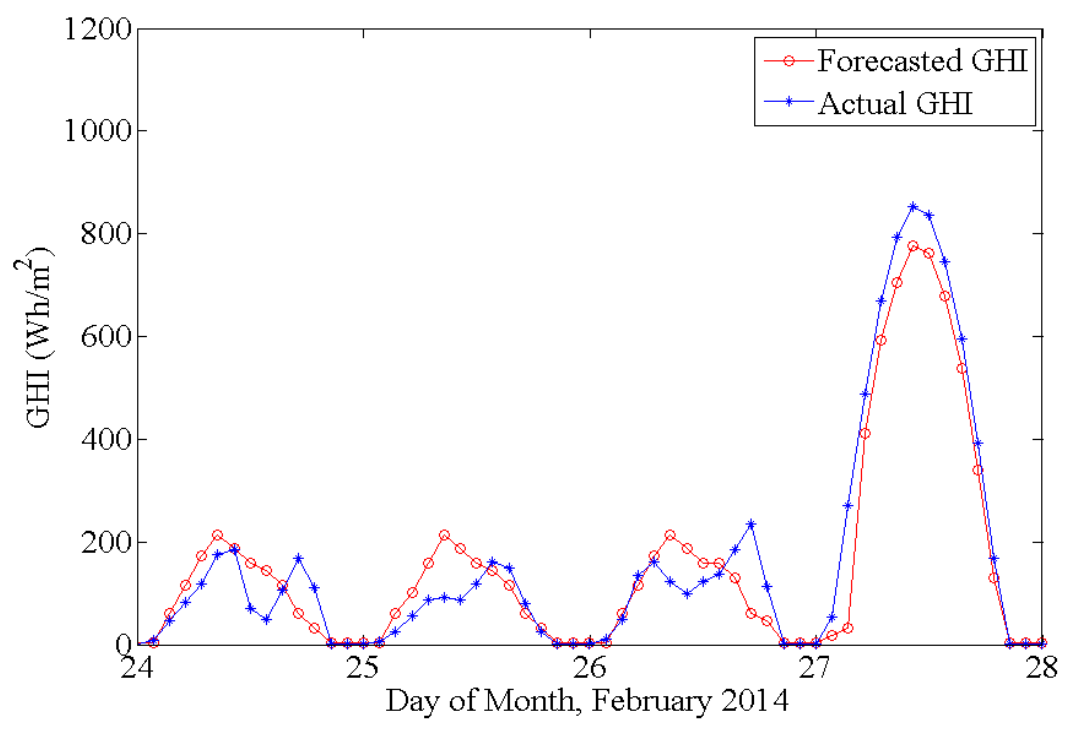

Fig. 3.8: Comparison between two days ahead predicted and actual GHI for 3 overcast and 1 clear day (type 3 ).

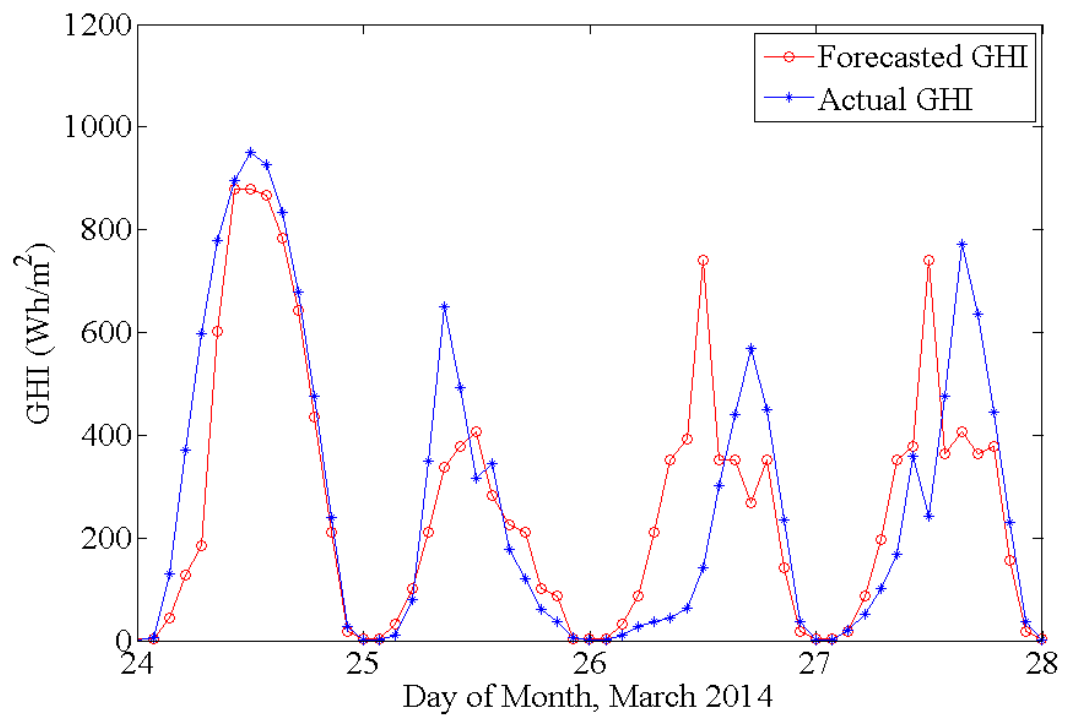

Fig.3.9: Comparison between two days ahead predicted and actual GHI for clear 1 clear and 3 partly cloudy days (type 4 ). 


\begin{tabular}{|c|c|c|c|c|}
\hline Weather type & $\begin{array}{c}r \\
(\%)\end{array}$ & $\begin{array}{c}\text { RMBE } \\
(\%)\end{array}$ & $\begin{array}{c}\text { MAPE } \\
(\%)\end{array}$ & $\begin{array}{c}\text { RRMSE } \\
(\%)\end{array}$ \\
\hline Type 1 & 99.82 & -1.49 & 2.85 & 2.93 \\
\hline Type 2 & 84.16 & -1.33 & 476.33 & 50.94 \\
\hline Type 3 & 96.86 & -3.4 & 280.17 & 29.45 \\
\hline Type 4 & 74.18 & -8.43 & 73.59 & 55.43 \\
\hline
\end{tabular}

Table 3.2: Summary of two days ahead prediction errors for 4 days.

It is worth comparing predicting performance of the proposed NB model (Table 3.2) against the approaches of previous studies (Table III) that might be helpful to understand the above MAPE nature. The existing prediction models are ANN [43] and MLP [56], which are mentioned in the introduction section.

\begin{tabular}{|c|c|c|c|c|c|}
\hline Methods & Weather type & $\begin{array}{c}r \\
(\%)\end{array}$ & $\begin{array}{c}\text { RMBE } \\
(\%)\end{array}$ & $\begin{array}{c}\text { MAPE } \\
(\%)\end{array}$ & $\begin{array}{c}\text { RRMSE } \\
(\%)\end{array}$ \\
\hline \multirow{3}{*}{ ANN } & Sunny & 98.77 & $\mathrm{x}$ & 9.45 & $\mathrm{x}$ \\
\cline { 2 - 6 } & Cloudy & 98.46 & $\mathrm{x}$ & 9.88 & $\mathrm{x}$ \\
\cline { 2 - 6 } & Rainy & 65.63 & $\mathrm{x}$ & 38.11 & $\mathrm{x}$ \\
\hline \multirow{2}{*}{ MLP } & Sunny & 99.45 & 12.97 & $\mathrm{x}$ & 38.26 \\
\cline { 2 - 6 } & Cloudy & 93.85 & 25.02 & $\mathrm{x}$ & 69.78 \\
\hline
\end{tabular}

Table 3.3: Summary of one day ahead prediction errors for 4 days.

ANN in Table 3.3 shows the MAPE of $38.11 \%$ for rainy days, which is the better result than the type 3 in the NB model result (MAPE of $280.17 \%$ ). However, regarding the $r$, the NB model shows the better values of $96.86 \%$ than that of ANN (65.63\%). Since the ANN could recognize the rainy days' pattern during 4 rainy days it might reduce the MAPE, but the pattern could not reflect the actual value's tendency. Contrary to this, the 
NB model (type 3) was able to follow the tendency of the mixed weather type for 4 days, but it provided overestimation for overcast days that result in the large MAPE. Strictly speaking, because of the different testing environment and metrics the two techniques ANN and MLP are not directly comparable with the proposed NB model. However, based on the predicting time scale (two days vs one day) and comparing 4 days' weather types (mixed weather types vs a specified weather type), the predicting performance of the NB model is acceptable regarding the results of ANN and MLP. Furthermore, with 4 days' mixed weather types test, the nature of MAPE may not be appropriate for monthly and mixed weather types forecasting evaluation, which is close to real-time weather changes.

\subsection{Wind Power Prediction Model}

As with the developed solar power prediction model, wind power output is modeled with the same methodology. For estimating the two days-ahead solar power prediction, the proposed NB model was performed with the forecasted weather variables since the predicted GHI is not included in weather forecast information. However, unlikely the GHI, the predicted wind speed is typically obtained from weather information. Therefore, with the same algorithm that is the proposed NB model, the relationship between forecasted wind speed and actual wind power is established.

Hourly observed wind speed from [33] is directly converted to power using theoretical turbine wind speed-power output function. Then these calculated wind powers are used in the proposed NB model with two-days-ahead forecasted wind speed [33] in order to establish the relationship between these two. Since the direct conversion, which 
transforms wind speed to wind power, and then building a model in wind power domain, instead of constructing a model in wind speed domain and then calculating wind power, can reduce the unnecessary information in a Markov transition matrix as in [63], before applying the proposed NB classifier, wind speed is directly transformed to theoretical wind power. 


\section{Chapter 4. Battery SOC Estimation}

This chapter investigates how the power output of renewable energy sources affect the design of a reliable energy storage model. Based on the balance of power output between PV, wind turbine, and BS, the energy states in an energy storage model are modeled using Markov chain. Due to the random nature of the power output, a stochastic process Markov chain is used.

\subsection{STEAdy STATE CONDITION}

\subsubsection{One-Step Transition Probability Matrix}

First, estimation of battery SOC is discussed based on the developed approach in [22], which proposed the Markov chain energy storage model in order to evaluate the relationship between power supply availability and battery bank capacity in steady state condition as shown in Fig. 4.1.

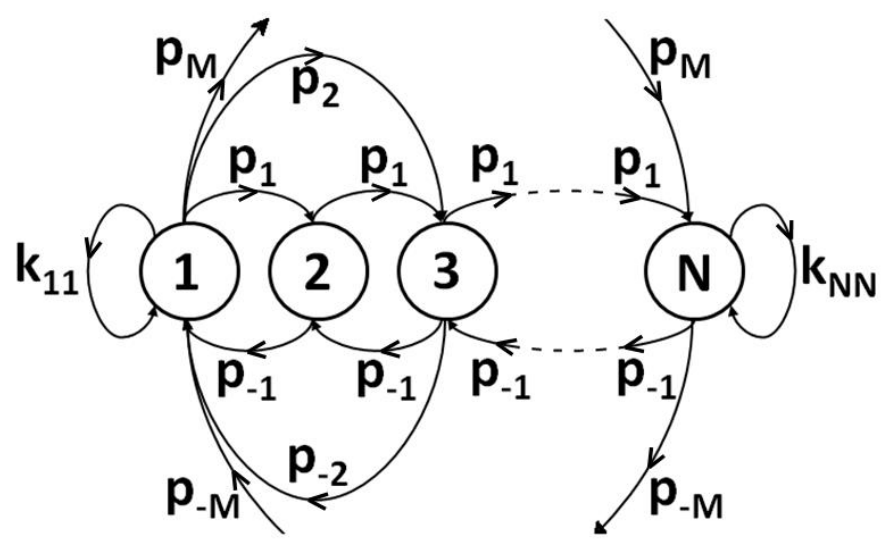

Fig. 4.1: State transition diagram for the battery bank SOC from [22]. 
This Markov chain model begins by determining net power Net, which is the sum of renewable sources' power output and BS power consumption (Chapter 2) as follows

$$
\text { Net }=\text { Solar }+ \text { Wind }-B S .
$$

With the net power profiles, a one-step transition matrix $\boldsymbol{P}$ in (4.2) [22] is constructed with several features: First, the transition matrix is a square matrix in which each row indicates the current energy state and each column represents the next energy state in the set Markov chain time steps. Second, each transition probability $p_{i}$ has a probability less than 1 so that the sum of any row must be equal to 1 . Third, diagonal entries except for first and last row represent the staying probabilities that are zero. Lastly, the special transition probabilities in (4.3) through (4.6) indicate that, beyond the battery bank capacity, the battery bank cannot be charged or discharged at any state at given Markov chain time step.

$$
\begin{gathered}
\boldsymbol{P}=\left[\begin{array}{ccccccc}
k_{11} & p_{1} & p_{2} & & & & \\
k_{11} & 0 & p_{1} & \cdots & & \vdots & \\
\hat{p}_{-2} & p_{-1} & 0 & & & & \\
& & & & 0 & p_{1} & \hat{p}_{2} \\
& \vdots & & \cdots & p_{-1} & 0 & k_{N N} \\
& & & p_{-2} & p_{-1} & k_{N N}
\end{array}\right]_{N \times N} \\
k_{11}=1-\sum_{i=1}^{M} p_{1}
\end{gathered}
$$




$$
\begin{aligned}
k_{N N} & =1-\sum_{i=1}^{M} p_{-1} \\
\hat{p}_{k} & =1-\left(\sum_{i=1}^{k-1} p_{i}+\sum_{i=1}^{M} p_{-i}\right) \\
\hat{p}_{-k} & =1-\left(\sum_{i=1}^{k-1} p_{-i}+\sum_{i=1}^{M} p_{i}\right)
\end{aligned}
$$

\subsubsection{Limiting Probabilities}

After a one-step transition matrix $\boldsymbol{P}$ is constructed with the transition probabilities $p_{i}$, there are several ways to solve the limiting probability vector $\boldsymbol{\pi}$. $\boldsymbol{\pi}$ signifies stationary probabilities of each energy state in the steady state battery operation. For example, with an assumption that there is a $8.1 \mathrm{kWh}$ capacity of battery bank and $\Delta=350 \mathrm{~W}$, limiting probabilities vector $\boldsymbol{\pi}$ is comprised of 24 limiting probabilities in the steady states. $\pi$ can be obtained in several ways, such as solving a transposed eigen-vector of $\boldsymbol{P}$, a system of linear equations, or a unique vector $\pi$. The third way is illustrated as follows. For any probability vector $\boldsymbol{v}$ and for large values of $n$, there is a unique limiting probability vector $\boldsymbol{\pi}$ from the result of the newly proposed Markov chain model:

$$
\begin{gathered}
\boldsymbol{\pi}=\lim _{n \rightarrow \infty} \boldsymbol{v} \cdot \boldsymbol{P}^{\boldsymbol{n}} \\
\boldsymbol{\pi}=\left[\begin{array}{lllll}
\pi_{1} & \pi_{2} & \cdots & \pi_{23} & \pi_{24}
\end{array}\right]_{24 \times 24} .
\end{gathered}
$$


Namely, (4.7) and (4.8) indicate that regardless of any initial SOC (vector $\boldsymbol{v}$ ), $\boldsymbol{v}$ converges to a fixed vector in the steady state when $n$ gets larger, which represents the expected probabilities of SOC in a steady state battery operation.

Limiting probabilities $\pi$ can also be used to obtain loss of load probability (LOLP) $\pi_{L} \cdot \pi_{L}$ indicates the probabilities of encountering a power deficiency in every discharging process at each energy state: in other words, the load demand is not completely fulfilled by renewable energy storage systems. $\pi_{L}$ is determined by summing the multiplying discharging transition probabilities $p_{-i}$ with the limiting probabilities as follows [22]:

$$
\pi_{L}=\sum_{i}\left[p_{-i} \sum_{j \leq i} \pi_{j}\right]
$$

where $i \in\{1, \cdots, 23\}$ that is each energy state and $\pi_{j}$ is each limiting probability.

Finally, the power supply availability in steady state condition can be obtained by subtracting $\pi_{L}$ in (4.9) from the total sum of probability 1 as follow

$$
A_{S S}=1-\pi_{L}
$$

where $A_{S S}$ is the availability in steady state condition which represents the probability that renewable sources coupled with an energy storage can supply power to loads. 


\subsubsection{Availability vs Capacity}

Under the assumption that the charging and discharging processes are linear, the capacity of the renewable energy storage system can be determined as

$$
C_{\text {bank }}=(N-1) \cdot \Delta \cdot T
$$

where $N$ is the total number of states in a battery bank and $T$ is the time step (time scale) in the Markov chain. From the previous results, the relationship between availability and capacity can be found by proving that the limiting probabilities depend on the capacity. Equation (4.8) indicates that the levels of limiting probabilities are determined by the levels of energy states, which will depend on the capacity of the battery bank. A larger battery bank capacity can handle a greater degree of variable power output with more energy transition states.

\subsubsection{Statistical Test for Markov Chain Model}

When Markov chain is used for many other applications, it is important to check whether the applications satisfy two Markov chain properties: dependence (4.12) and stationary (4.13). If the successive events are dependent of each other and the transition probabilities in Markov chain are independent of time, they follow a first order Markov chain. The studies [44] and [64] already demonstrated the first order Markov chain properties using hourly solar irradiance (GHI) wind speed variable. Therefore, before 
constructing the Markov transition probabilities matrix of net power (PV + wind - BS), the testing of Markov chain properties are evaluated.

1) Dependency Test

Equation (4.12) indicates Markov chain dependency test [38]

$$
\alpha=2 \sum_{i, j}^{N} n_{i j} \ln \frac{p_{i j}}{p_{j}}
$$

where $N$ is the total number of battery states, $n_{i j}$ is the number of transition from state $i$ to $j, p_{i j}$ is transition probability $\left(p_{i}\right.$ in (4.2)), and $p_{j}$ is marginal probability. If the dependency test with $(N-1)^{2}$ DF (degree of freedom) asymptotically follows $\chi^{2}$ distribution, it can be interpreted that the subsequent events are independent.

2) Stationary Test

Stationary test is defined as [38]

$$
\beta=2 \sum_{s}^{S} \sum_{i, j}^{N} n_{i j}(s) \ln \left(\frac{p_{i j}(s)}{p_{i j}}\right)
$$


where $S$ is the number of subintervals in the test period and $\boldsymbol{n}_{\boldsymbol{i j}}(\boldsymbol{s})$ is the elements of the tally matrix at $s$ th subinterval. If the stationary test (4.13) with $\boldsymbol{N}(\boldsymbol{N}-\mathbf{1})(\boldsymbol{S}-\mathbf{1})$ DF has a $\chi^{2}$ it can be interpreted that the Markov chain is stationary.

\subsubsection{Sizing Battery Bank (Energy Storage)}

Before the relationships between battery bank capacity and availability are studied, it is important to note that the data timeline is divided into two parts as shown in Fig. 4.2: battery bank capacity and real-time operation evaluation. Moreover, a BS power consumption (2.6) is calculated based on the average daily traffic profile in Europe from [47]. The probabilities of battery bank SOC in the steady state operation are estimated based on $\pi_{i}$ in [22]. However, each $\pi_{i}$ has a small value in the given number of battery bank SOC so it might be helpful to separate the states by three levels in order to understand the property of SOC for reliable battery bank utilization. In the case of the $8.1 \mathrm{kWh}$ battery bank capacity for configuration $1(\mathrm{C} 1), 24 \mathrm{SOC}$ can be separated into three levels of limiting probabilities: the sum of SOC less than or equal to \#5 (bottom), between \#6 and \#22 (middle), and greater than or equal to \#23 (top). Since discharging below DOD level has a permanent reduction on the battery's life, the bottom level is determined according to the bottom $20 \%$ in each of the 24 states. For the top level, top $10 \%$ in the 24 states is determined (also, this methodology is applied for $\mathrm{C} 2$ in the same way). With a maximum power of about $11.5 \mathrm{~kW}(6 \times 240 \mathrm{~W}+10 \mathrm{~kW})$ for configuration 1 and $4.8 \mathrm{~kW}(20 \times 240 \mathrm{~W})$ 
for $\mathrm{C} 2$, the renewable sources with an energy storage system in each configuration supply power to an LTE BS.

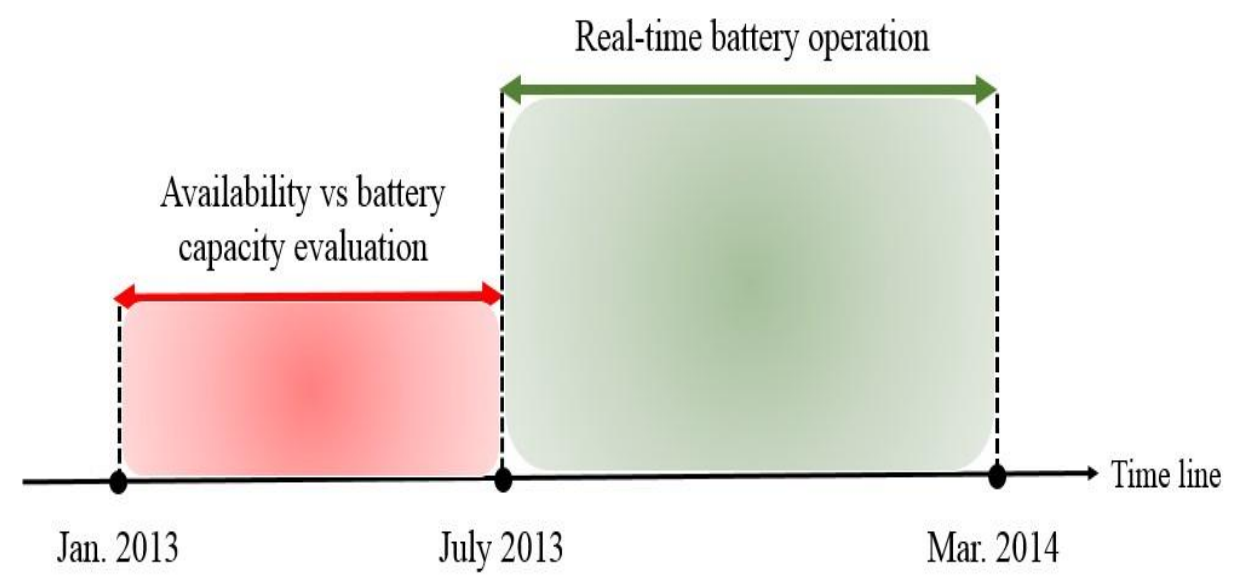

Fig. 4.2. Data time line: power supply availability evaluation (red) and real-time operation period (green).

Table 4.1 indicates the test statistics for the proposed Markov chain model in Fig. 4.1 and (4.2) regarding on the dependency in (4.12) and stationary test (4.13). As shown in Table 4.1 it can be interpreted that the successive net power events in the proposed Markov chain are dependent because both configurations $(\mathrm{C} 1$ and $\mathrm{C} 2)$ indicate the statistics of $2.05 \times 10^{5}$ and $2.36 \times 10^{5}$ that are greater than Chi-square values of 583.61 and 792.92 . Furthermore, for the stationary test, it can be interpreted that the proposed Markov process is stationary with the 12 subintervals $(S=12)$ because the statistics of $4.6 \times 10^{3}$ and $5.28 \times$ $10^{3}$ are less than the Chi-square values of $1.8 \times 10^{4}$ and $8.53 \times 10^{3}$. 


\begin{tabular}{|c|c|c|c|c|c|}
\hline Configuration & Test & DF & $\begin{array}{c}\text { Significance } \\
\text { level }\end{array}$ & $\begin{array}{c}\text { Chi-square } \\
\text { value }\end{array}$ & Statistic \\
\hline \multirow{2}{*}{ C1 } & Dependency & 529 & 0.05 & 583.61 & $2.05 \times 10^{5}$ \\
\cline { 2 - 6 } & Stationary & 6072 & 0.05 & $1.8 \times 10^{4}$ & $4.6 \times 10^{3}$ \\
\hline \multirow{2}{*}{ C2 } & Dependency & 729 & 0.05 & 792.92 & $2.36 \times 10^{5}$ \\
\cline { 2 - 6 } & Stationary & 8316 & 0.05 & $8.53 \times 10^{3}$ & $5.28 \times 10^{3}$ \\
\hline
\end{tabular}

Table 4.1: The proposed Markov chain dependency and stationary test for two configurations.

Fig. 4.3 and 4.4 shows the two pie-charts of limiting probabilities $\pi_{i}$ that describe the three levels' percentage distribution for each configuration. When the battery banks in two cell sites are compared in terms of the bottom level, $33 \%$ of configuration 1 (C1) indicates the better result than $57 \%$ of configuration 2 (C2), but the both levels still indicate the large proportion in $\pi_{i}$. The bottom level results can be interpreted that the two configuration would have the risk probabilities of $33 \%$ and $57 \%$ at any given battery operation point in time which may affect the battery life reduction. Once the $\pi_{i}$ is calculated, the loss of load probabilities $\pi_{L}$ is determined to be $14 \%$ and $30.36 \%$ and finally, the power supply availability for BSs are estimated to be $86 \%$ and $69.64 \%$, which implies more is needed to be done, such as increase in generation or battery bank capacity, for the configurations to be more sustainable power supply network system. 


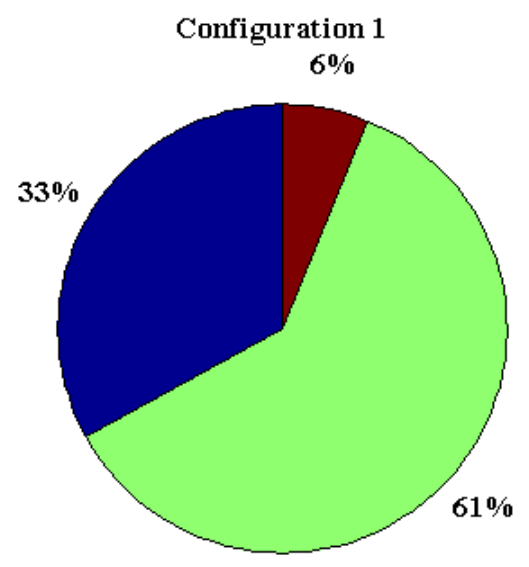

Bottom $\square$ Middle $\square$ Top

Fig. 4.3: 8.1 kWh battery bank capacity with 24 SOC states.

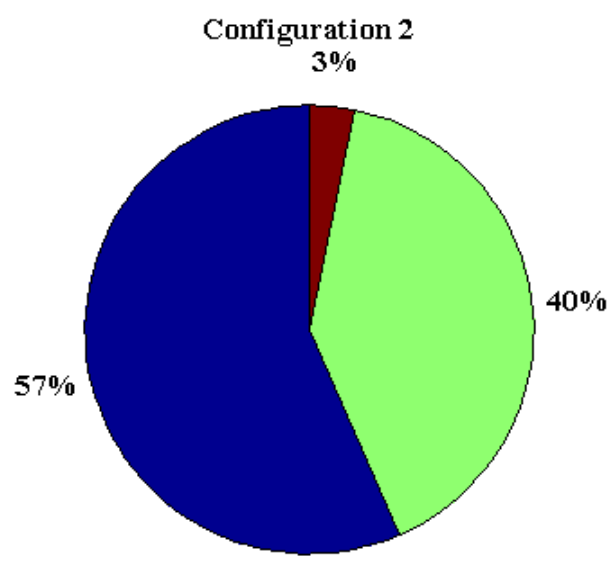

Bottom $\square$ Middle

Top

Fig. 4.4: 9.45 kWh battery bank capacity with 28 SOC states. 
Fig. 4.5 shows how the availability values affect the design of battery capacity in two configurations. The results show that slopes of both curves are expected to increase asymptotically at a given large capacity, which is over $18 \mathrm{kWh}$ for $\mathrm{C} 1$ and over $12 \mathrm{kWh}$ for $\mathrm{C} 2$. That is, the power supply availability would not improve at some point due to the limit of renewable sources' capacity. This can also be interpreted as, a significant increase in the slope at some point does not necessarily suggest a suitable battery capacity for a given target availability. One of the most interesting things about designing a site configuration in SWA is determining the size of energy storage. As shown in Fig. 4.3 and 4.4 , the battery capacity of $8.1 \mathrm{kWh}$ for the $\mathrm{C} 1$ and $9.45 \mathrm{kWh}$ for the $\mathrm{C} 2$ are located around knee point on the curves, which might be properly designed given the generation capacity. Therefore, instead of increasing the battery SOC states (capacity) in (4.11), managing load power consumption would provide a better solution to increase the power supply availability. 


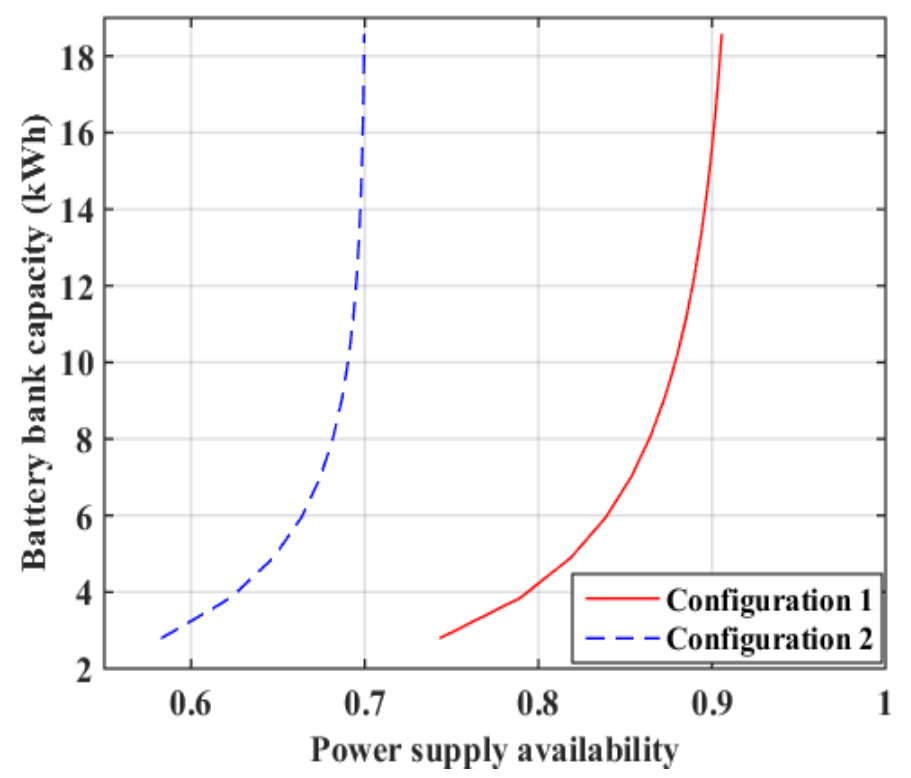

Fig. 4.5: Sizing battery bank capacity (SOC) depending on target power supply availability.

Since cost is an important factor in design and operating cellular networks, cost of power sources and energy storage is estimated from the configurations considered. Configuration 1, C1 consist of 6 MX60-240 PV modules, an Excel $10 \mathrm{~kW}$ wind turbine and an energy storage of $8.1 \mathrm{kWh}$, whereas in Configuration 2, C2 consist of $20 \mathrm{MX} 60$ $240 \mathrm{PV}$ modules and an energy storage of $9.45 \mathrm{kWh}$. Fig. 4.6 shows a simplified cost chart depending on the battery bank capacity. To compare the cost of $\mathrm{C} 1$ and $\mathrm{C} 2$, a battery cost of $150 \$$, a PV module of $350 \$$ and a wind turbine of $30 \mathrm{k} \$$ are assumed based on the discussed models in Chapter 2. 


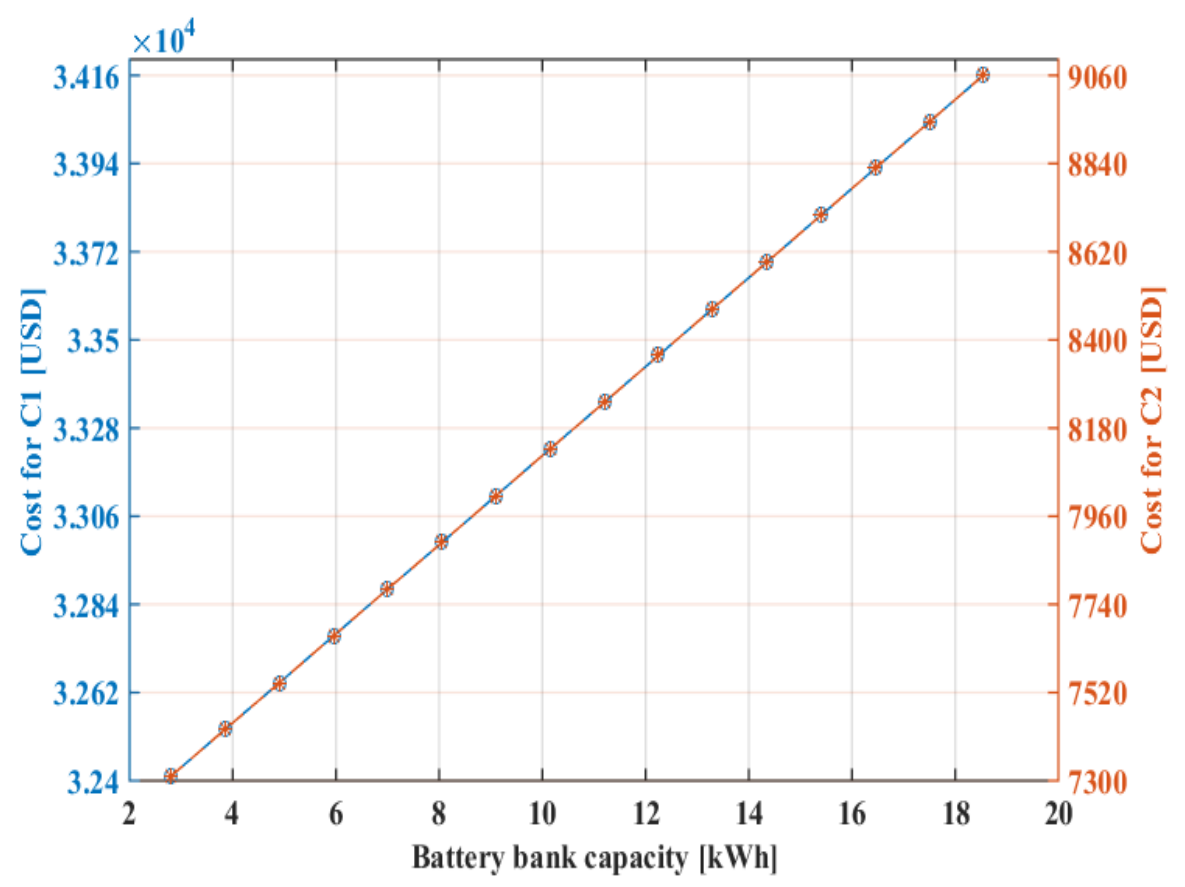

Fig. 4.6: Two configurations' cost analysis with different battery bank cost.

Fig. 4.7 shows the results of power supply availability depending on the battery bank capacity. The difference in the results obtained in Fig. 4.5 and Fig. 4.7 can be explained as following. BSs' power consumption reduction (2.7) is performed as an average aspect for long-term process (steady-state operation) in Fig. 4.7 whereas the traffic shaping is not considered in Fig. 4.5. It is worth to noticing that the traffic shaping factor $\sigma$ has a range of between 0.3 and 1 where the maximum traffic shaping of 0.3 and no traffic-shaping of 1 . This process starts by calculating the net power (4.1) with the traffic shaping factor of 0.9 for $\mathrm{C} 1$ and 0.5 for $\mathrm{C} 2$. Then, transition probability matrix (4.2) is constructed and new limiting probabilities (4.7) are obtained. For the case with a lower traffic shaping for $\mathrm{C} 2(\sigma=0.5)$, the results show that the power supply availability at the 
given capacity of $9.45 \mathrm{kWh}$ is 0.997 . This is an improved value compared to 0.676 in Fig. 4.5. The results are shown in Fig. 4.7 from which it can be observed that the availability values are different for $\mathrm{C} 1$ and $\mathrm{C} 2$ and they depend on the battery bank capacity.

Comparing the results of $\mathrm{C} 1$ and $\mathrm{C} 2$ in Fig. 4.7, the following can be observed. Although $\mathrm{C} 1$ is operated with little shaping $(\sigma=0.9)$, it can be operated to power BSs more sustainable. This is because of diverse power sources presented in C1 [28]. The results also show the limited generation capacity of the sources in $\mathrm{C} 2$.

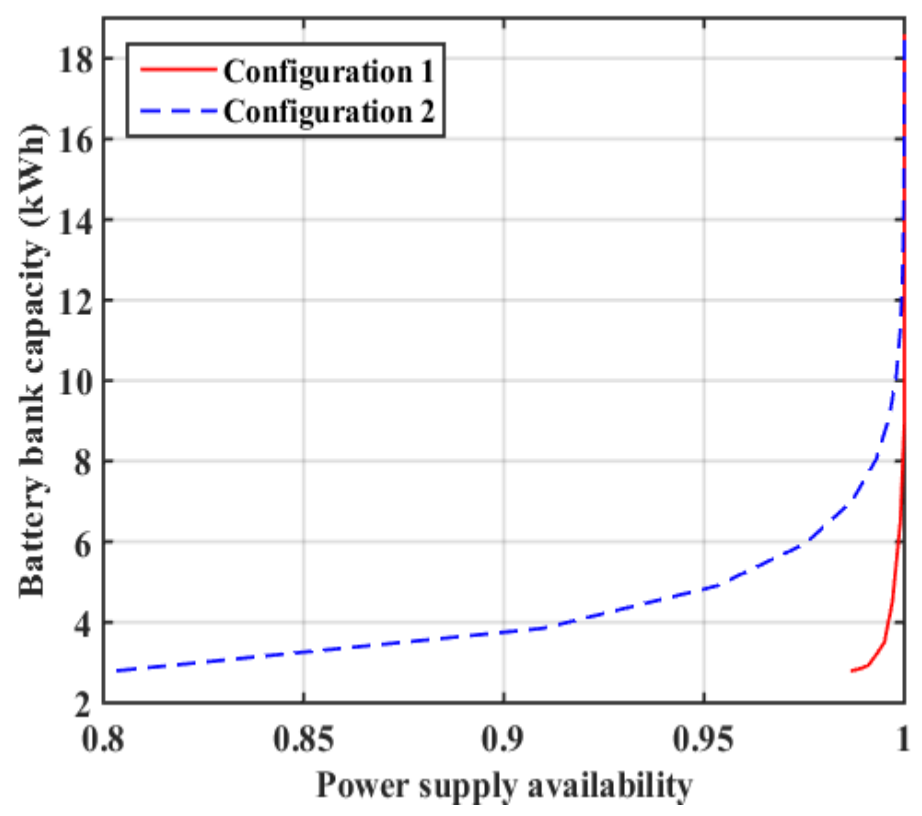

Fig. 4.7: Sizing battery bank capacity (SOC) with traffic shaping that is considered as a long-term process $\left(\sigma_{t}=0.9\right.$ for $\mathrm{C} 1$ and 0.5 for $\left.\mathrm{C} 2\right)$. 


\subsection{REAL Time CONDITION}

Similar to (4.1), it is possible to define the time-based net power $\operatorname{Net}_{t}$ as

$$
\operatorname{Net}_{t}=\text { Solar }_{t}+\text { Wind }_{t}-B S_{t}
$$

In the previous section, the Markov chain model was applied to estimate the battery transition from one SOC to another [22]. However, different from the study [22], which proposed estimating the limiting probabilities $\boldsymbol{\pi}_{\boldsymbol{i}}$ (expected probabilities of SOC in a steady state battery operation) that may be appropriate for the process in a long period sense, this section investigates how a real-time battery SOC is estimated.

This process begins by calculating the net power profiles up to $M$ hours in the future $\left(N e t_{t+1}, \cdots, N e t_{t+M}\right)$ based on the proposed NB model in Chapter 3. The integer $M$ is determined corresponding to battery bank capacity [48] and these profiles are used to calculate the net power transition probabilities $p_{i}$. Then, $M$-step net power transition probability matrix $\boldsymbol{P}^{\boldsymbol{M}}$, which includes $M$ hour-ahead battery SOC transition information, is constructed in order to determine the battery state probability vector,

$$
v_{t+M}=v_{t} P^{M}
$$

where $\boldsymbol{v}_{\boldsymbol{t}}$ is the measured battery SOC at time $t$. Therefore, the $\boldsymbol{v}_{\boldsymbol{t}+\boldsymbol{M}}$ represents the probability distribution of the battery bank SOC at time $t+M$ as follows

$$
\boldsymbol{v}_{\boldsymbol{t}+M}=\left[v_{1, t+M}, v_{2, t+M}, \cdots, v_{N-1, t+M}, v_{N, t+M}\right]_{1 \times N}
$$


where each $v_{i, t+M}$ represents the battery bank state probability. Once the $\boldsymbol{v}_{\boldsymbol{t}+\boldsymbol{M}}$ in $(4.15)$ is calculated, the maximum probability value in $\boldsymbol{v}_{\boldsymbol{t}+\boldsymbol{M}}$ is set as 1 and is selected as the maximum likelihood battery bank SOC at time $t+M$ so that the low level of SOC would be prevented by the stored energy in battery bank and adequate load control. For example, when the variables in (4.11) are assumed as $N=28, \Delta=350$ and $T=1$ hour, the required battery bank would be $9.45 \mathrm{kWh}(=27 \times 350 \mathrm{~W} \times 1$ hour $)$ and if the battery SOC at time $t$ represents fully charged state $\left(\boldsymbol{v}_{\boldsymbol{t}}=[0, \cdots, 1]_{1 \times 24}\right)$, then the $M$ hour ahead state vector $\boldsymbol{v}_{\boldsymbol{t}+\boldsymbol{M}}$ is estimated by multiplying the $24 \times 24$ transition matrix $\boldsymbol{P}^{\boldsymbol{M}}$.

Prediction uncertainty is the main reason in using the $M$ hours-ahead battery bank SOC estimation. As demonstrated in Chapter 3, the proposed NB model shows good agreement between actual and prediction values regarding on the MBE and RMBE metric, so prediction uncertainty can be addressed with the proposed NB model.

Table 4.2 shows a summary of the statistical values for renewable energy prediction for $\mathrm{C} 1$ and $\mathrm{C} 2$. Based on 24-hour basis, the mean value of hourly observed renewable energy, MBE, and RMBE are evaluated. As demonstrated in Chapter 3, the proposed NB model for $\mathrm{C} 1$ and $\mathrm{C} 2$ also show good result for the MBE in (3.15) and RMBE in (3.18) metric, which indicate the hourly average error of $-38.31 \mathrm{Wh}$ and $42.7 \mathrm{Wh}$ regarding on the hourly average actual renewable energy of $1.071 \mathrm{kWh}$ and $794.09 \mathrm{Wh}$. In other words, $\mathrm{C} 1$ and $\mathrm{C} 2$ indicate the $\mathrm{RMBE}$ of $-3.58 \%$ and $5.3 \%$ that represent the proportion of errors when they are compared with the hourly average energy production for 8 months. 


\begin{tabular}{|c|c|c|c|}
\hline Configurations & Mean $\left[\mathrm{Wh} / \mathrm{m}^{2}\right]$ & MBE $[\mathrm{Wh}]$ & RMBE $(\%)$ \\
\hline $\mathrm{C} 1$ & 1071.4 & -38.31 & -3.58 \\
\hline $\mathrm{C} 2$ & 794.09 & 42.7 & 5.38 \\
\hline
\end{tabular}

Table 4.2: Statistics of predicting renewable energy in two configurations. 


\section{Chapter 5. Integrated Power Management in SWA}

This chapter introduces how the balance of power between renewable sources' generation and BSs' consumption is managed depending on predicted renewable energy and stored energy state in battery banks. Specifically, real-time traffic shaping and power sharing algorithm are investigated, which affect the battery SOC in order to not only increase the use of renewable sources, but also decrease the excessive battery discharging. In order to simplify the calculations, this study deals with two BSs in a cellular network that operate according to a high-level controller in [65]. Then, mean down time (MDT) and battery throughput, which can quantify the critical infrastructure resiliency and battery life in years, are evaluated with 8-month battery operation simulation.

\subsection{Sustainable Wireless Area (SWA)}

\subsubsection{System Architecture}

This dissertation proposes a sustainable wireless area (SWA) concept, which is designed by interconnecting neighboring BSs in a dc microgrid configuration as shown in Fig. 5.1 (originally developed by A. Kwasinski). With the coverage radius of 250 meters for each BS, seven BSs are clustered as a SWA in an urban area (Chapter 2). In the SWA architecture, the arrangement of renewable sources can be centered or distributed corresponding to each cell site's configuration ( $\mathrm{C} 1$ and $\mathrm{C} 2)$ in order to address the relatively large physical footprint. Fig. 5.2 (provided by A. Kwasinski) also shows the selfcontained SWA electrical scheme, which has a battery bank in each cell site. Different to 
conventional cell sites where the battery banks are used for back up power system during the power outage in cellular networks, in the SWA, the battery banks play a pivotal role such as managing renewable sources' power output variability, sharing power between cell sites, and storing sufficient energy from renewable sources. The goal of the SWA architecture is to be self- sufficient the majority of time from renewable sources and only in a minority of times from conventional power grid.

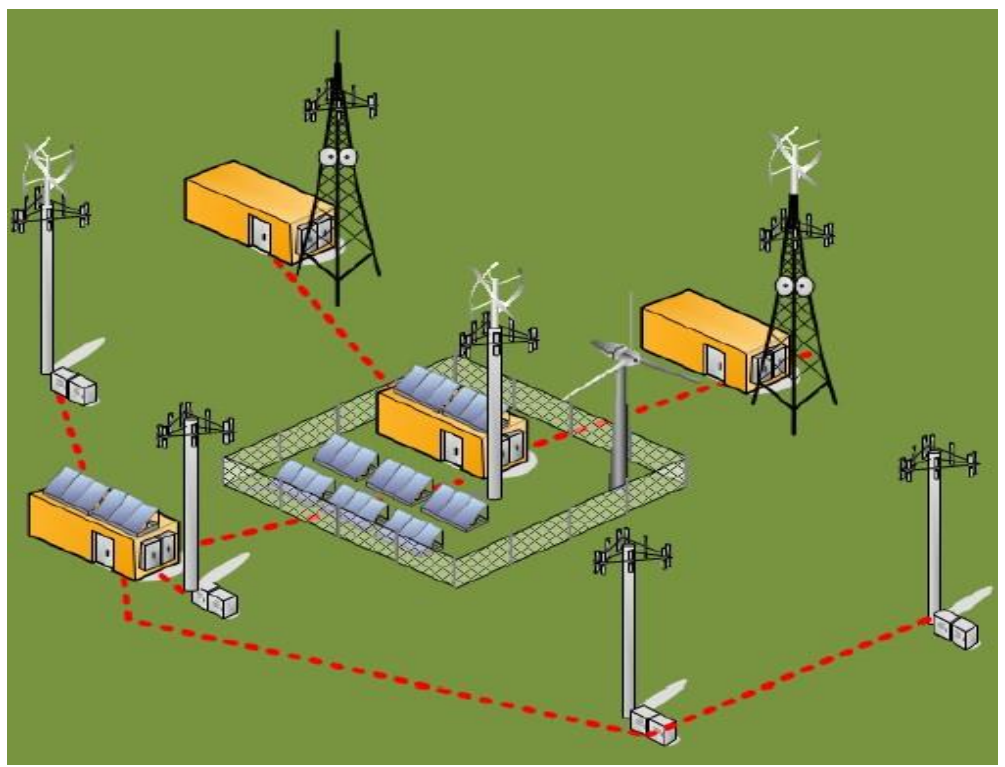

Fig. 5.1: Proposed SWA architecture that comprises of seven base stations with renewable source and storage system from [46]. 


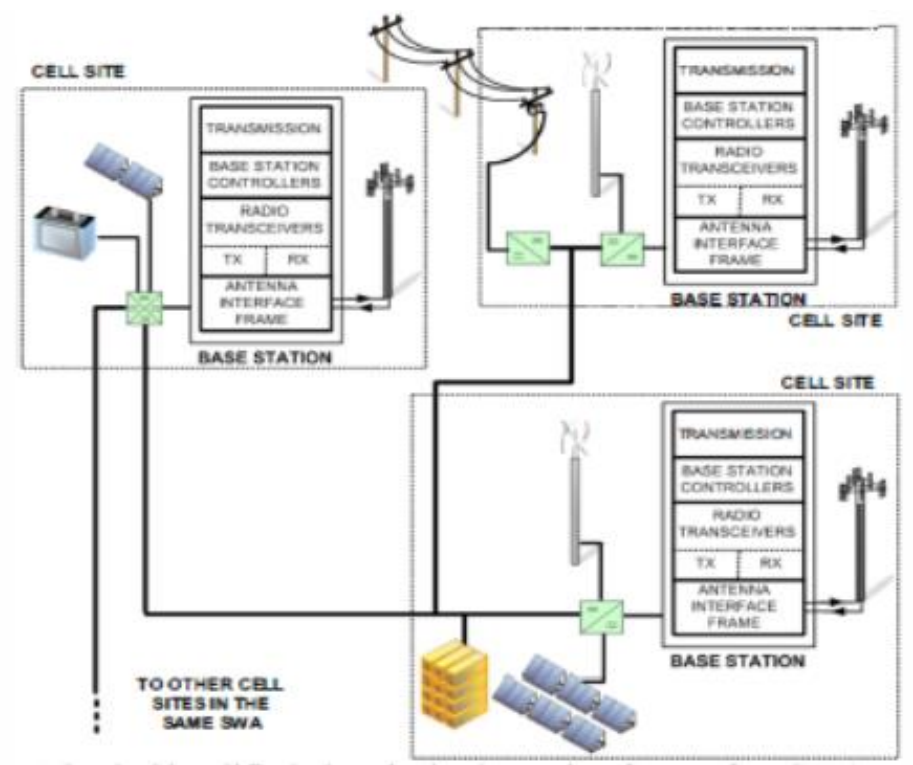

Fig. 5.2: Electrical scheme of a SWA from [65].

\subsubsection{High Level Controller}

The self-contained SWA operates the integrated traffic-power management by the command of controller in [65]. As shown in Fig. 5.3 (originally developed by A. Kwasinski) the controller consists of two levels in which the top level determines the SWA operation condition by collecting all information from renewable sources, battery banks, and BSs. The typical bottom level controller operates based on the high-level commandsvoltage and current level for power source converter and traffic level for BSs-but the bottom level controller can act autonomously during the failure in the communication link between two levels. Also, the output information at bottom level is transmitted to the top level in order to control the dynamin behavior of the SWA operation. This feedback loop 
(interactions between two levels of the electric controller) is achieved every alternative hour. Therefore, this dissertation focuses on the high-level controller that controls the traffic-power management in order to realize the reliable network system that has sustainable power supply availability.

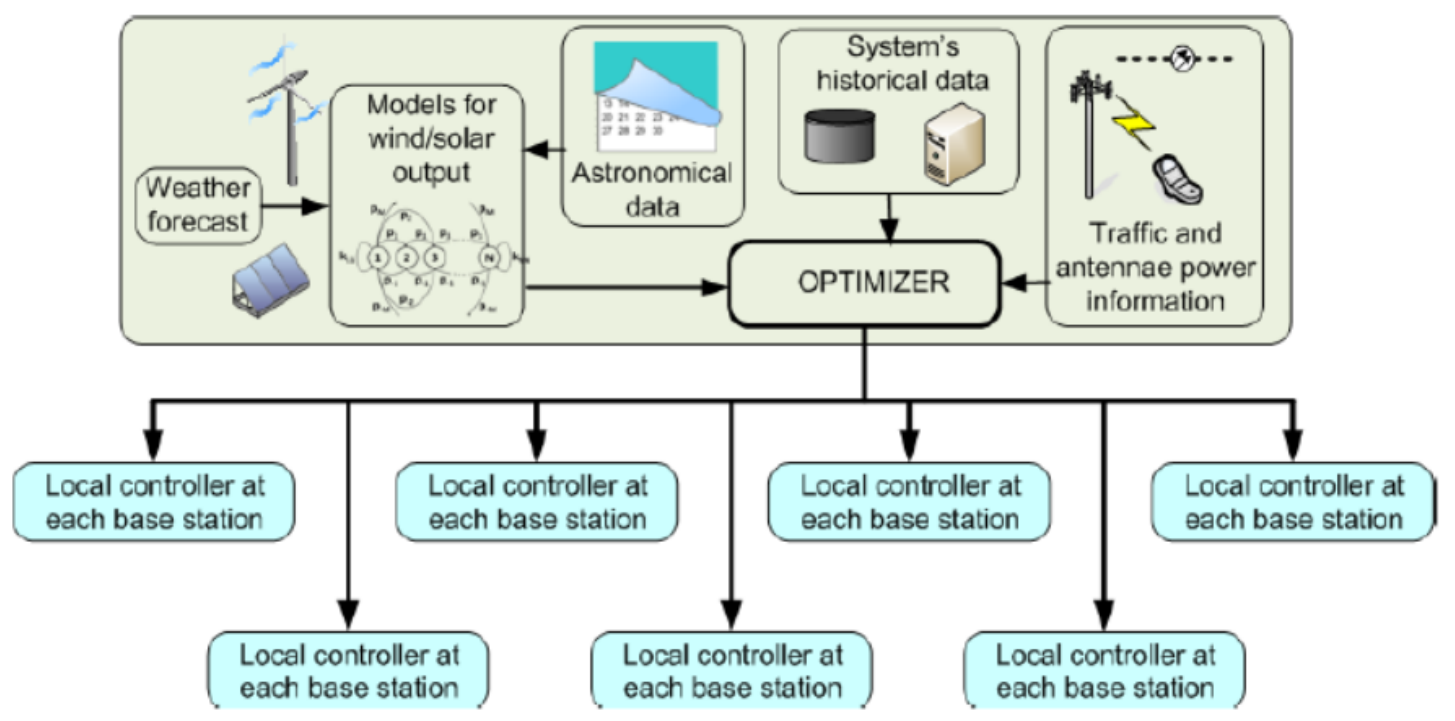

Fig. 5.3: Hierarchical controller architecture from [65].

\subsection{INTEgrated TrafFic-PoWer CONTROL}

This section investigates how the high-level controller manages the integrated traffic-power in the SWA. Based on the predicted renewable energy and battery SOC, the control factor in (2.6) and cell sites' operational condition are determined in the high-level controller. 


\subsubsection{Traffic Shaping Strategies}

Based on (2.6) and (4.7), the possible scenarios for choosing control factor $\sigma_{t+1}$ that represents traffic load shaping can be organized as shown in Table 5.1. As mentioned earlier, since the control strategies depend on the forecasted renewable energy and the battery SOC, all cases are considered to provide proper strategies. The first step to obtain the control factor $\sigma_{t+1}$ is to determine $\Delta s_{\max }$, which is not only the upper limit of the logistic function, but also the condition for initiating the control strategies. As defined in [48], $\Delta s_{\max }$ represents the maximum value of $N e t_{t+1}$ that is set with the maximum traffic shaping $\left(\sigma_{t+1}=0.3\right)$ so $\Delta s_{\max }$ can also be used as Event 1 trigger condition in Table 5.1, which indicates that the $\Delta s_{\max }$ is greater than 0 or not. Once Event 1 is determined, each control strategy under Event 1 can be chosen in 3 cases in order to choose $\sigma_{t+1}$. This study focuses only on the real-time Markov chain model under the condition of $\Delta s_{\max }>0$ in Case 2, since the logistic function was studied in [48] and the other cases are straightforward.

As shown in Table 5.1, the real-time iterative process using a Markov chain model in Fig. 5.4 is activated when the maximum likelihood SOC at time $t+M$ in (4.15) is less than $S_{\text {lim }}\left(S O C_{t+M}<S_{\text {lim }}\right)$, where $S_{\text {lim }}$ represents a lower limit for battery SOC. $S_{\text {lim }}$ can be determined based on the battery technology such as lead-acid or lithium-ion battery. However, if the maximum probability state in $\boldsymbol{v}_{\boldsymbol{t}+\boldsymbol{M}}$ in (4.15) indicates less than $S_{\text {lim }}$, control factor $\sigma_{t+1}$ will be adjusted (decreased) and the iterative Markov chain process in Fig. 5.4 is repeated until the maximum probability state is equal to the $S_{\text {lim }}$. Therefore, 


\begin{tabular}{|c|c|c|c|}
\hline Event 1 & \multicolumn{2}{|c|}{$\Delta s_{\max } \leq 0$} & $\Delta s_{\max }>0$ \\
\hline Case 1 & \multicolumn{2}{|c|}{$\begin{array}{c}\text { If } S O C_{t}<S_{l i m} \\
\sigma_{t+1}=0.3\end{array}$} & 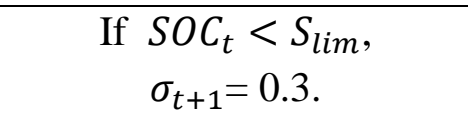 \\
\hline \multirow[t]{6}{*}{ Case 2} & \multicolumn{2}{|c|}{ If $S_{\text {lim }} \leq S O C_{t}<S_{c}$} & If $S_{\text {lim }} \leq S O C_{t}<S_{c}$ \\
\hline & $S O C_{t}$ & $\sigma_{t+1}$ & Logistic function for $\sigma_{t+1}$. \\
\hline & {$[0.8,0.9)$} & 0.8 & \\
\hline & {$[0.7,0.8)$} & 0.7 & But if $S O C_{t+M}<S_{\text {lim }}$, \\
\hline & $\vdots$ & $\vdots$ & real-time Markov chain \\
\hline & {$[0.3,0.4)$} & 0.3 . & for $\sigma_{t+1}$ \\
\hline Case 3 & \multicolumn{2}{|c|}{ 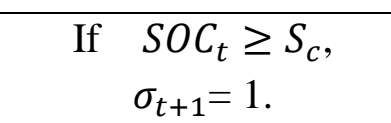 } & $\begin{array}{l}\text { If } \quad S O C_{t} \geq S_{c}, \\
\sigma_{t+1}=1\end{array}$ \\
\hline
\end{tabular}

Table 5.1: Traffic shaping scenario.

using the real-time Markov model, the $\sigma_{t+1}$ is controlled in order to satisfy the condition $S O C_{t+M}>S_{\text {lim }}$. If the real-time Markov chain model fails to satisfy the condition, the $\sigma_{t+1}$ is set as 0.3 which represents the maximum traffic shaping.

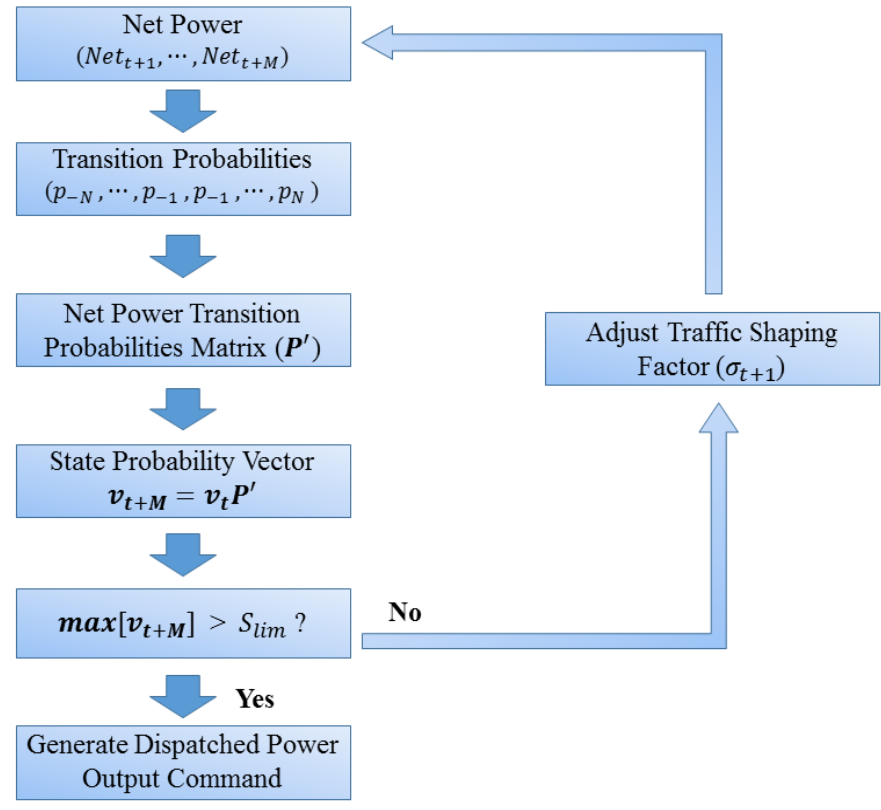

Fig. 5.4: A real-time iteration algorithm using Markov chain. 
Fig. 5.5 shows an example of above explanation. Thanks to the control strategies and $M$ hours-ahead SOC estimation, the excessive battery discharges (under $S_{\text {lim }}$ ) and \#1 state (fully discharged) are avoided (red curve) from the no traffic shaping (blue curve) as shown in Fig. 5.5 (a). Fig. 5.5 (b) shows the control factor $\sigma_{t+1}$, which is determined corresponding to control strategies in Table I. Fig. 5.5 (c) shows the renewable energy, which the predicted values (red circle) tend to follow the actual renewable energy (blue star).

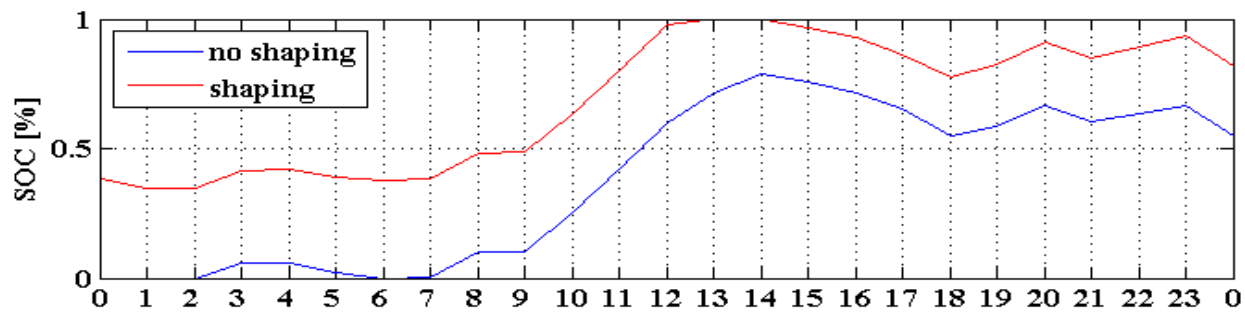

(a)

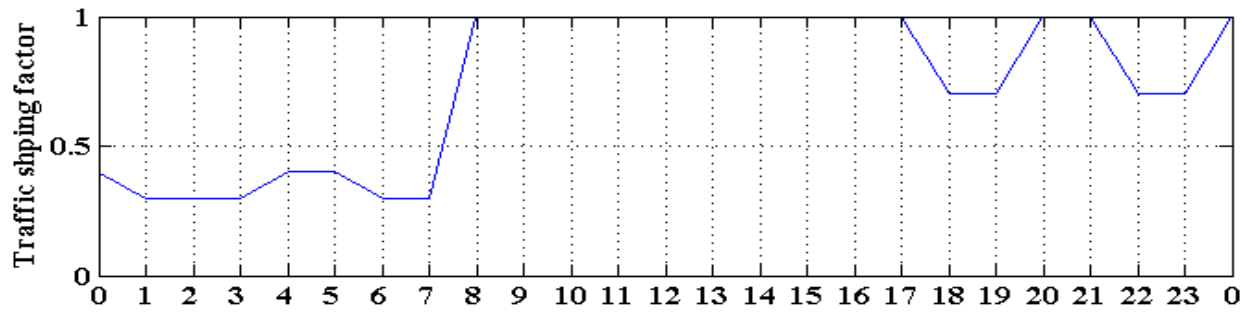

(b)

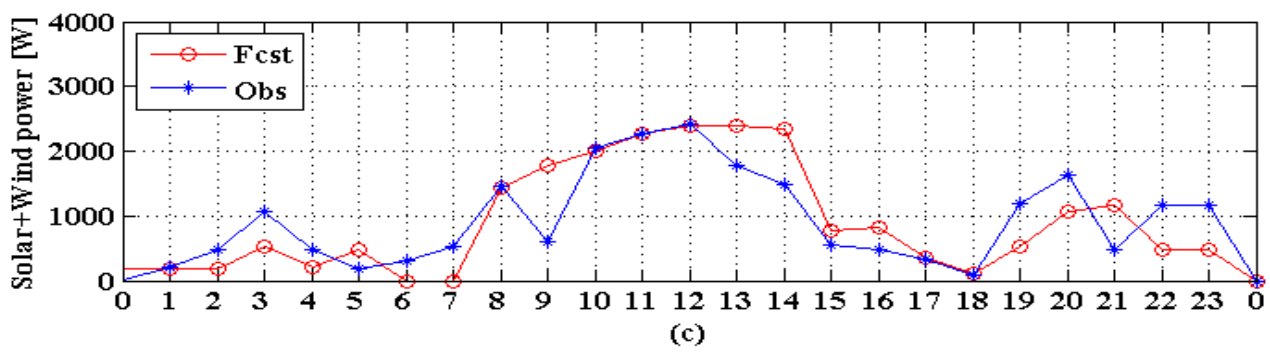

Hour of day, September 4th 2013

Fig. 5.5: One day results for traffic shaping strategies in Sep. $4^{\text {th }}$ 2013: (a) SOC, (b) traffic shaping factor, and (c) actual and forecasted renewable energy. 


\subsubsection{Power Sharing Strategies}

First, the two BSs in a SWA are operated independently with the proposed control strategies in Table 5.1. However, when one battery bank SOC at $t+1$ is estimated to be lower than the lower limit $\left(S O C_{t+1}<S_{\text {lim }}\right)$ while the other battery bank is estimated to be sufficient energy, or one battery bank SOC at $t+1$ is expected to be higher than fully charged $\left(S O C_{t+1}>1\right)$ while the other battery bank is not expected to be fully charged, the battery banks are controlled to share power in the SWA. This power shaping aims to prevent battery deep discharges and make the SWA to be more resilient network system. The power sharing strategies in Table 5.2 are activated after the traffic load shaping scenarios in Table 5.1 are applied. Since the power transfer between battery banks in the SWA causes the charge-discharge losses by the battery round-trip efficiency, in order to reduce the round-trip losses the power sharing is operated when the two events in Table 5.2 happened only after the traffic shaping. For multiple cell sites, the average high level battery bank SOC at time $t+1$ is determined as follows:

$$
S O C_{H, t+1}=\frac{\sum_{h=1}^{n} S O C_{h, t+1}}{n}, \quad 1 \leq n \leq 7
$$

where the $S O C_{h, t+1}$ represents the expected high level battery bank SOC that is greater than 0.6 and the $n$ indicates the number of the high level battery bank SOC in SWA. The expected average low level battery SOC, $S O C_{L, t+1}$, is also defined with $S_{l i m}$ on the same 


\begin{tabular}{|c|c|}
\hline Event 2 & $\begin{array}{l}\text { When } S O C_{H, t+1} \leq 1, \\
\left(C_{\text {bank }, L, t+1} \cdot S_{\text {lim }}-S O C_{L, t+1}\right) \cdot \eta_{\text {batt }} \text { is transferred to } \\
\text { each } S O C_{l, t+1} \text { from each } S O C_{h, t+1} \text {. }\end{array}$ \\
\hline Event 3 & $\begin{array}{l}\text { When } S O C_{H, t+1}>1 \text {, } \\
\left(S O C_{H, t+1}-C_{\text {bank,H,t+1}}\right) \cdot \eta_{\text {batt }} \text { is transferred to } \\
\text { each } S O C_{l, t+1} \text { from each } S O C_{h, t+1} \text {. }\end{array}$ \\
\hline
\end{tabular}

Table 5.2: Power sharing scenarios.

plan as (5.1). Table 5.2 shows the power sharing scenarios that are explained above. Similar to the traffic shaping strategies in Table 5.1, the power sharing strategies begin by determining the event, which the $S O C_{H, t+1}$ is greater than 1 or not. In Table 5.2 $C_{b a n k, L, t+1}$ and $C_{b a n k, H, t+1}$ represent the average low and high level battery bank capacity at time $t+l$ and $\eta_{\text {batt }}$ represents the battery charge-discharge efficiency.

\subsection{SWA RESILIENCY}

The resiliency of CIs (e.g., cellular networks) can be improved by the use of renewable sources, especially during the occurrence of destructive natural disasters since the generation of renewable sources does not have lifelines [28], [66]. The study [67] discusses the metric of CIs resiliency which how the degree of dependence between CIs can be characterized by quantifying the resiliency metric. From reliability theory availability of a system or portion of a system is calculated by [67]

$$
A=\frac{M U T}{M U T+M D T}
$$


where mean up time (MUT) represents the expected time that the system is in a required operation and mean down time (MDT) represents the expected failure time that the system is not working or is in state of repair. Again, the MDT is used to express repair rate $\mu$ as follows

$$
\mu=\frac{1}{M D T}
$$

where $\mu$ represents how fast the CI can be recovered from the failure. Therefore, the MDT can be considered as the metric that quantifies the CIs resiliency.

From the view of MDT in [67], this dissertation investigates the evaluation of timebased CIs resiliency based on the $N e t_{t}$ in (4.7). Since the renewable sources in C1 and C2 do not depend on lifelines, in order to characterize the degree of dependence among the cell sites ( $\mathrm{C} 1$ and $\mathrm{C} 2)$, namely how the dependence influences the resiliency of each cell site, the $N e t_{t}$ is considered to represent the concept of the MUT $\left(N e t_{t}>0\right)$ and MDT $\left(\mathrm{Net}_{t} \leq 0\right)$ for the SWA resiliency evaluation. In addition, since the MDT (or $\mu$ ) is related with the logistical management in human-centered aspect [67], the proposed traffic shaping and power sharing strategies (logistic management) will be simulated in the next section in order to look for that how the strategies have an effect on the time of MDT $N e t_{t} \leq 0$. 


\subsection{BATTERY BANK LIFETIME MODEL}

Under various conditions, a battery operation may be affected when it processes the charge and discharge cycle. Benchmarking project in [68] defined the battery stress factors, which causes an aging process by chemical damage mechanisms. In terms of stress factors, this dissertation focuses on the battery throughput $(\mathrm{kWh})$ factor in order to see how the traffic shaping and power sharing control affect the battery life time.

Fig. 5.3 shows the expected number of cycles to failure (CTF) and throughput corresponding to DOD for a US 250E XC2 battery.

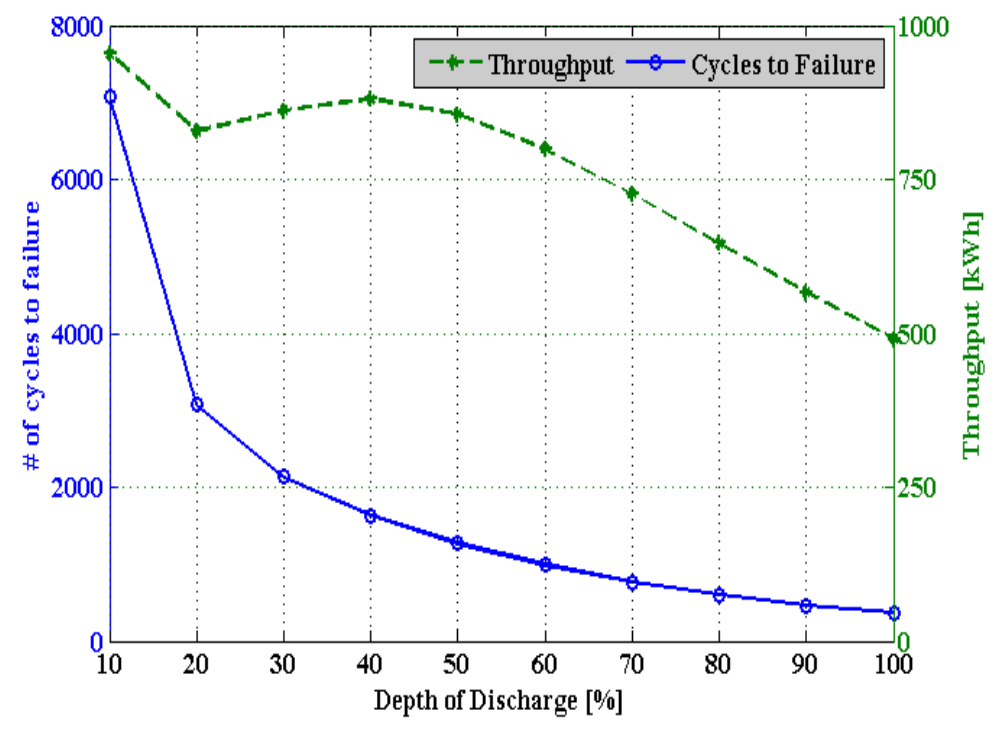

Fig. 5.6: Expected life cycle \& throughput vs DOD for deep-cycle battery model for US $250 \mathrm{E}$ XC2.

The CTF and throughput curves are estimated by the manufacturer data in [69] which is measured at a 20 -hr rate with $225 \mathrm{Ah}$ and $6 \mathrm{~V}$. The CTF curve is found by fitting a double exponential curve 


$$
C T F=\alpha_{1} \cdot e^{a_{2} \cdot x}+\alpha_{3} \cdot e^{a_{4} \cdot x}
$$

where $\alpha_{i}$ is the fitting coefficients $\left(\alpha_{1}=3.388 \times 10^{4}, \alpha_{2}=-0.223, \alpha_{3}=4415\right.$, and $\alpha_{4}=-$ 0.025) and $x$ is the percentage DOD values. Based on the CTF curve, the throughput is calculated as

$$
T_{b a t t}=\frac{\sum_{i=1}^{n}\left(D O D_{i} \cdot C T F_{i} \cdot C\right)}{n}
$$

where the subscript $i$ is a trial number, $C T F_{i}$ is the cycles to failure at $D O D_{i}$ which is determined by rainflow counting method in [70], and $C$ is a nominal battery capacity $(1.35$ $\mathrm{kWh}=6 \mathrm{~V} \times 225 \mathrm{Ah}$ ). While the CTF curve shows the dependency on the DOD which the CTF decreases as the DOD increases, the $T_{\text {batt }}$ curve indicates less dependence on the DOD. For this reason, HOMER [71] suggested a way to calculate the life time of battery bank based on the assumption that the $T_{\text {batt }}$ is independent of the DOD. Thus, the battery bank life in year is calculated as follows

$$
\text { Life } e_{\text {bank }}=\frac{T_{\text {batt }} \cdot N}{T_{\text {bank }}}
$$

where $N$ is the number of batteries in a battery bank and $T_{b a n k}$ is the total throughput in a year. 


\subsection{SiMULATION RESULTS}

The first evaluation is to investigate the local operation in the SWA that how the integrated traffic-power control affects the battery bank SOC in terms of the resiliency and battery life. In this case, two cell sites are operated independently based on the strategies in Table 5.1. Then the results of independent operation are compared with the power sharing results, which are operated by the strategies in Table 5.1 and 5.2.

Fig. 5.4 (a) and (b) show the two cumulative distribution functions (CDFs) performing with the control algorithms in Table 5.1. Along with the different settings of control factor such as no traffic shaping, with traffic shaping (Table 5.1), and maximum traffic shaping $\left(\sigma_{t+1}=0.3\right)$, the battery bank operations in two configurations are simulated. This dissertation assumes that when the expected battery bank SOC is fully discharged (\#1), BS will be connected to grid or diesel generator to be powered. First, when the two cell sites are operated without the traffic shaping, the CDFs indicate the time availability of $82.82 \%$ for $\mathrm{C} 1$ and $64.54 \%$ for $\mathrm{C} 2$, respectively. Similar to the previous steady state operation results, which indicate the availability of $86 \%$ for $\mathrm{C} 1$ and $69.64 \%$ for $\mathrm{C} 2$, respectively, this 8-month real-time simulation also shows that $\mathrm{C} 1$ has the better time availability than $\mathrm{C} 2$. When the traffic shaping (load control) is applied, the available time proportion in the two configurations indicates $94.17 \%$ and $79.22 \%$, which $11.35 \%$ (about 28 days) and $14.68 \%$ (about 36 days) are increased. In other words, when the MDT (\#1) is calculated in days - by multiplying the time proportion by 243 days ( 8 months) the proportion of MDT is reduced from about 42 days (C1) and 86 days (C2) to 14 days and 50 days. If the maximum traffic shaping is applied for the whole test period, the time 
availability can be increased to $97.02 \%$ and $83.01 \%$ for the two configurations, but this aggressive setting results in maximum reduction in video quality and increase in data delay. 


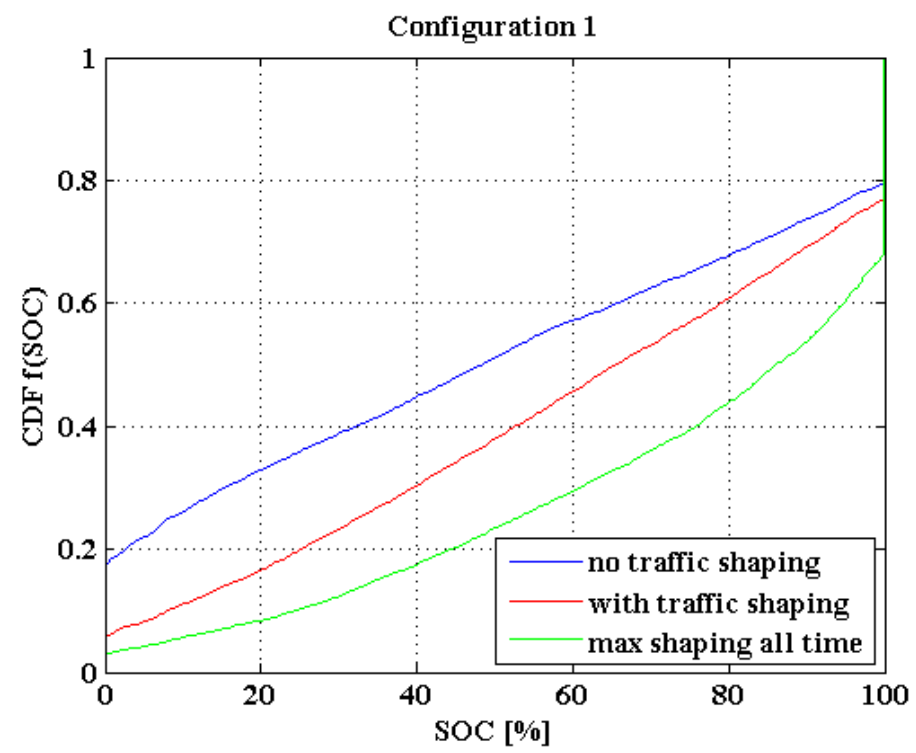

(a)

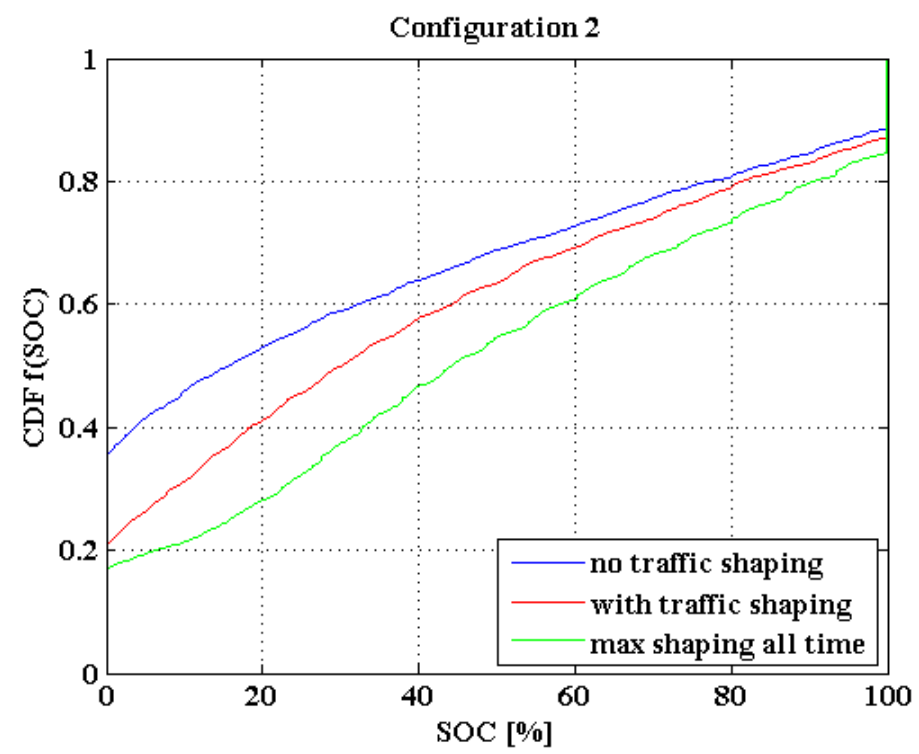

(b)

Fig. 5.7: Cumulative distribution functions (CDFs) for the battery SOC with different operating conditions for 8 months: configuration 1 (a) and configuration 2 (b). 
Fig. 5.5 (a) and (b) show the stacked bar plots that are same results with the CDFs in Fig. 5.4. As the same way in the three levels of $\boldsymbol{\pi}_{\boldsymbol{i}}$, the number of battery bank SOC is divided into three levels - bottom (blue), middle (light green), and top (red) levels - in order to clearly understand time proportion at each level. The results show that the bottom level in $\mathrm{C} 1$ decreases from $32.87 \%$ to $16.6 \%$ when the traffic shaping algorithms in Table I are applied. That is, the time proportion of $16.27 \%$ (about 40 days) is improved from the bottom level that permanently affects battery life reduction. Also, the middle level increases from $40.76 \%$ to $52.54 \%$ and top level increases from $26.37 \%$ to $30.86 \%$. In the case of $\mathrm{C} 2$, the bottom levels indicate $52.91 \%$ and $41 \%$ for without and with traffic shaping. Although the time proportion of $11.91 \%$ (about 29 days) is improved from the bottom to middle level due to the effect of the real-time control, it still shows the significant time proportion in the bottom level. 


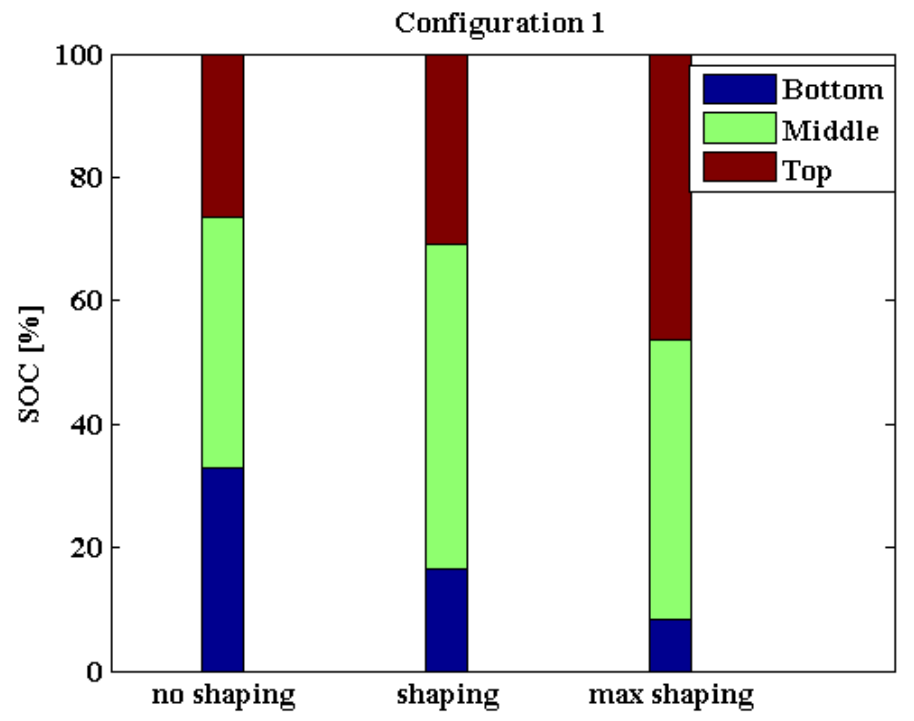

(a)

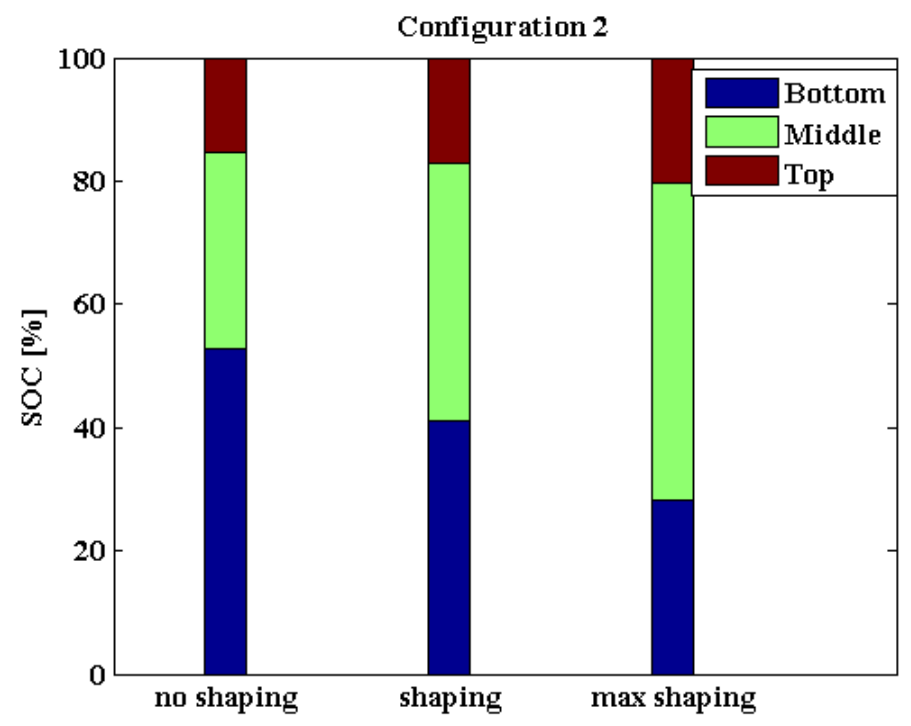

(b)

Fig. 5.8: Percentage of three level of SOC with different operating conditions for 8 months: configuration 1 (a) and configuration 2 (b). 
Table 5.3 shows the results of local battery bank operation that draws a comparison between no traffic shaping and traffic shaping. The average of DOD, number of cycles and MDT in days are evaluated from the 8-month simulation as well as the battery bank CTF, throughput, and life in year are modeled in order to evaluate the effectiveness of the traffic shaping in each cell site. In the case of battery bank life model, $\mathrm{C} 1$ indicates the better performance than $\mathrm{C} 2$ in terms of the longer CTF and higher throughput. However, in the matter of battery bank life, $\mathrm{C} 2$ has the longer life time of 1.11 year than $\mathrm{C} 1$ of 0.93 year. From the evaluated items in Table 5.3, we can draw a conclusion that the battery bank charge-discharge rate in $\mathrm{C} 1$ has the faster than $\mathrm{C} 2$. The charge-discharge rate is the one of the major stress factor that changes the rate of chemical damage mechanism in a battery bank [68]. As shown in 8-month simulation results, the average DOD of C2 indicates significantly large value of $98.89 \%$ (close to fully discharged SOC at each cycle) while it has the lower number of cycle of 225 than $\mathrm{C} 1$ of 633 . That is, the battery bank in $\mathrm{C} 2$, which is only coupled with the PV modules, operates with the deep cycle and low discharge rate. This characteristics might be evident that when the PV modules are the only power generation resources for the BS, the continuous discharges occur during night time. For the case of traffic shaping in Table 5.3, the results are discussed with the following power sharing in SWA. 


\begin{tabular}{|c|c|c|c|}
\hline & Evaluation item & $\begin{array}{c}\mathrm{C} 1 \\
\text { no shaping/shaping }\end{array}$ & $\begin{array}{c}\mathrm{C} 2 \\
\text { no shaping/shaping }\end{array}$ \\
\hline \multirow{2}{*}{$\begin{array}{c}\text { 8 months } \\
\text { simulation }\end{array}$} & Avg. DOD [\%] & $64.37 / 51.55$ & $98.89 / 96.32$ \\
\cline { 2 - 4 } & \# of cycles & $633 / 647$ & $225 / 230$ \\
\cline { 2 - 4 } & MDT [days] & $42 / 14$ & $86 / 50$ \\
\hline \multirow{2}{*}{$\begin{array}{c}\text { Battery bank } \\
\text { life model }\end{array}$} & CTF & $892 / 1224$ & $375 / 401$ \\
\cline { 2 - 4 } & Throughput[kWh] & $767.6 / 846.7$ & $499.2 / 518.8$ \\
\cline { 2 - 4 } & Life in year & $0.93 / 1.02$ & $1.11 / 1.15$ \\
\hline
\end{tabular}

Table 5.3: Comparison between no traffic shaping and traffic sharing in two configurations.

For the power sharing evaluation, this dissertation focuses on the time proportion in bottom level that is directly related to a battery life. Fig. 5.6 shows the CDFs that the BSs are controlled by the strategies in Table 5.1 and 5.2 for 8 months. In Fig. 5.6, C2 (blue line) shows the noticeable increase around $S_{\text {lim }}$ (30\% of SOC) where the energy transfer happens from $\mathrm{C} 1$ to $\mathrm{C} 2$ corresponding to the strategies Table 5.2. Since in the case of $\mathrm{C} 2$, the time proportion under $S_{\text {lim }}$ indicates $49.88 \%$ for the previous traffic shaping case (value in parenthesis) in Table 5.4, the noticeable increase around $S_{\text {lim }}$ can be explained as the results of Event 2 in Table 5.2 (also, all other values within parenthesis in Table 5.4 stand for the values from the previous traffic shaping results in the local operation). When the power sharing is activated, MDT and the time proportion at bottom level are significantly improved in $\mathrm{C} 2$ while $\mathrm{C} 1$ is also improved slightly. For the measure of time availability, C2 indicates the time availability of $91.55 \%$ from $79.22 \%$ and for the measure of time proportion at bottom level, $\mathrm{C} 2$ is improved from $41 \%$ to $25.74 \%$ for 8 months simulation. 


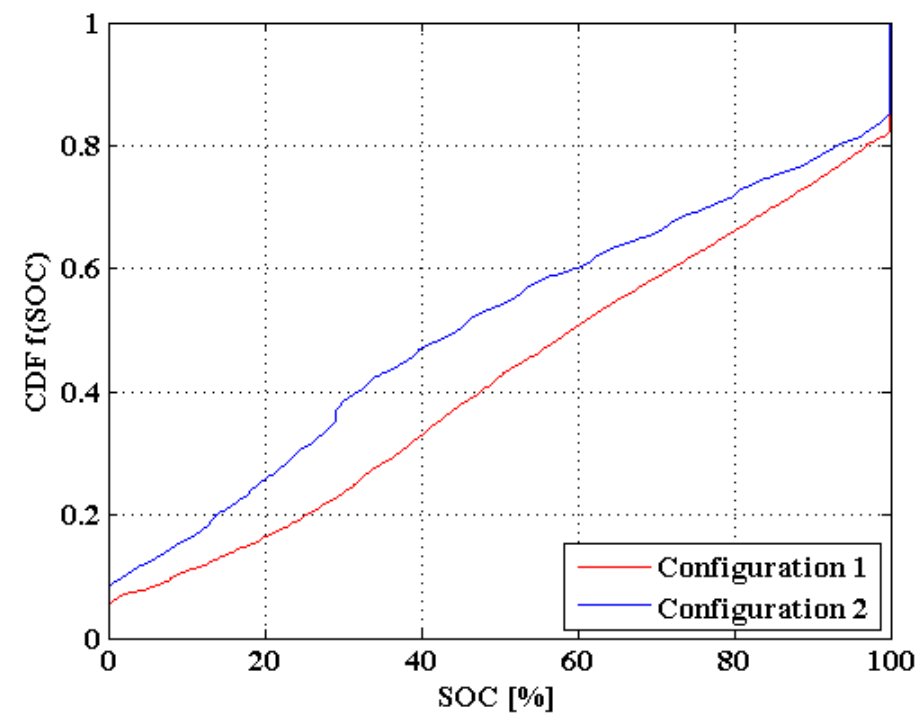

Fig. 5.9: Cumulative distribution functions (CDFs) for the power sharing in SWA.

\begin{tabular}{|c|c|c|c|}
\hline & & $\begin{array}{c}\mathrm{C} 1 \\
\text { time proportion [\%] }\end{array}$ & $\begin{array}{c}\mathrm{C} 2 \\
\text { time proportion [\%] }\end{array}$ \\
\hline \multirow{3}{*}{ SOC [\%] } & 0 & $5.62(5.83)$ & $8.45(20.78)$ \\
\cline { 2 - 4 } & $\leq 20(\mathrm{DOD})$ & $16.41(16.6)$ & $25.74(41)$ \\
\cline { 2 - 4 } & $\leq 30\left(S_{\text {lim }}\right)$ & $23.53(23.2)$ & $38.44(49.88)$ \\
\cline { 2 - 4 } & $\leq 90\left(S_{c}\right)$ & $73.85(69.14)$ & $77.71(82.97)$ \\
\cline { 2 - 4 } & 100 & $17.83(23.08)$ & $14.81(12.74)$ \\
\hline
\end{tabular}

Table 5.4: SOC results for power sharing in SWA.

Table 5.5 also indicates the significantly improved results in $\mathrm{C} 2$ while $\mathrm{C} 1$ keeps the similar results. The MDT in C2 shows the most noticeable change when the power sharing strategies are activated. The MDT is effectively improved more than twice from 50 days to 20 days but it is more remarkable when the power sharing is compared with the no traffic 
shaping of 86 days in Fig 5.4 (b). That is, the MDT of three cases in C2 is significantly reduced in the order of no traffic shaping, local traffic shaping, and power sharing in SWA which indicate 86, 50, and 20 days. Thanks to the control strategies in Table 5.1 and 5.2, not only the SWA resiliency is increased, but also the battery banks operations are improved in terms of the MDT and battery life.

\begin{tabular}{|c|c|c|c|}
\hline \multirow{2}{*}{$\begin{array}{c}\text { 8 months } \\
\text { results }\end{array}$} & Evaluation item & $\begin{array}{c}\mathrm{C} 1 \\
\text { no sharing/sharing }\end{array}$ & $\begin{array}{c}\mathrm{C} 2 \\
\text { no sharing/sharing }\end{array}$ \\
\cline { 2 - 4 } & Avg. DOD [\%] & $51.55 / 52.48$ & $96.32 / 72.94$ \\
\cline { 2 - 4 } & MDT of cycles & $647 / 784$ & $230 / 383$ \\
\hline $\begin{array}{c}\text { Battery life } \\
\text { model } \\
\text { evaluation }\end{array}$ & Throughput[kWh] & $14 / 14$ & $50 / 21$ \\
\cline { 2 - 4 } & Life in year & $1224 / 1204$ & $401 / 593$ \\
\hline
\end{tabular}

Table 5.5: Comparison between local traffic shaping and power sharing in two configuration. 


\section{Chapter 6. Conclusions}

Renewable energy sources are one of the most effective ways to reduce carbon emission and increase resiliency in cellular networks. However, as the power output of renewable sources is expected to be variable, it is valuable to study the prediction of renewable sources' power output. Moreover, it is also beneficial to study the control scheme of battery bank SOC, which can mitigate the power output variability in cellular networks. This dissertation discusses the design and operation of SWAs, which include renewable sources (solar and wind), energy storage, and BS and traffic-power management algorithms for their operation.

First, hourly renewable energy prediction using the proposed NB model was investigated which can predict up to two days ahead. Publicly available weather observation and forecast data were used as training sets and input values in the model. A key contribution of this study is to reduce the GHI overlapping classification by partitioning daily intervals into subsets. By limiting the classification range into one hour, the GHI variation can be mitigated which the NB model can follow the actual values' trend under various weather condition. The proposed NB model is fairly simple in that it requires small training data (less than two month) and uses only four weather variables (temperature, relative humidity, dew point, and sky coverage), whereas the prediction accuracy certainly was reasonable compared to the recent other predicting models.

For the assessment of prediction error, this dissertation contains discussion concerning MAPE error test criterion, which is unsuitable for interpreting real-time 
weather changes. In other words, MAPE might address several specific weather types, such as sunny, rainy, or cloudy days that the forecasting patterns can be trained under consistent weather condition. Moreover, this dissertation demonstrates that the proposed NB model can be used for the estimating battery bank SOC based on the MBE and RMBE metric which measure the tendency of the renewable energy that is over-estimated or underestimated given the testing period.

For an eight months period with hourly resolution (14 hours per day for GHI prediction), the proposed NB model provides prediction results with statistical values that are RMBE of $2.73 \%$, RRMSE of $41.7 \%$, and a correlation coefficient of $86.33 \%$ with GHI mean of $333.04 \mathrm{Wh} / \mathrm{m}^{2}$. For the mixed weather types, clear 4 days represent RMBE of $-1.49 \%$, MAPE of $2.85 \%$, RRMSE of $2.93 \%$, and a correlation coefficient of $99.85 \%$, which are considerably matched with the actual GHI.

Second, this dissertation proposes integrated traffic-power control algorithms that can effectively increase the power supply availability in the SWA only from the use of renewable energy sources; hence the SWA resiliency and battery life were improved. The integrated traffic-power control begins by modelling of renewable sources, BS, and energy storage from the actual solar irradiance, wind speed, and daily traffic profiles. Also, this dissertation proposes the two ways to estimate the battery bank SOC using Markov chain: steady-state and real-time way. The former one was used for sizing energy storage capacity while the latter one was used for the integrated traffic-power management in the high-level controller. Based on the predicted renewable energy and battery bank SOC information, the high-level controller determines the appropriate set points for the BSs operation in the 
SWA. In the local operation, the battery bank SOCs are separately controlled using the iterative real-time Markov chain model to prevent the battery life reduction. In the global operation, the power is shared in the SWA in order to increase the resiliency (hence availability) by making up the power deficiency in the local operation.

Two cell sites' configurations in the SWA are then compared in the view of resiliency and battery bank life based on the battery bank SOC CDF. When the two cell sites operate separately, the time availability proportion indicates $82.82 \%$ of $\mathrm{C} 1$ and $64.54 \%$ of $\mathrm{C} 2$ for 8 months. However, with the traffic shaping, the time availability indicates $94.17 \%$ of $\mathrm{C} 1$ and $79.22 \%$ of $\mathrm{C} 2$, which increase $11.35 \%$ (28 days) for $\mathrm{C} 1$ and $14.68 \%$ (36 days) for $\mathrm{C} 2$ from the MDT. More importantly, the time availability of C2 shows up to $91.55 \%$ when the power sharing in SWA is activated. This approach is novel in that, the battery bank, which shows better performance in the local operation (here, $\mathrm{C} 1$ ), is not significantly affected by the battery bank in C2. Indeed, when the power sharing is operated, C1 indicates slightly improved time availability from $94.17 \%$ to $94.38 \%$ while other factors such as CTF, throughput, and life in year maintain the similar values.

Lastly, the proposed model and algorithms can be expanded to various applications. For example, the proposed battery bank SOC models can assist system planners and operators to design and operate the battery bank efficiently with the estimated SOC information. Moreover, power consumption reduction can be realized using demandresponse (load control) during times of daily peak demand, which allows end-users to contribute to reduce carbon emission. In terms of educational value, more importantly, this dissertation helps to improve integrated thinking that includes the knowledge of power 
systems, communication systems and computing systems, which is currently needed in smart-grids. 


\section{References}

[1] W. Vereecken, W. Van Heddeghem, D. Colle, M. Pickavet, and P. Demeester, "Overall ICT Footprint and Green Communication Technologies," in ISCCSP, 2010, pp. 1-6.

[2] M. Pickavet, W. Vereecken, S. Demeyer, P. Audenaert, B. Veermeulen, C. Develder, D. Colle, B. Dhoedt, and P. Demeester, "Worldwide energy needs for ICT: the rise of power-aware networking," International Symposium on Advanced Networks and Telecommunication Systems (ANTS'08), Bombay, India, Dec. 2008.

[3] W. Vereecken et al., Power consumption in telecommunication networks: overview and reduction strategies, IEEE Communications Magazine, vol. 49, no 6, 62-69, 2011.

[4] Amit Kumar, Tanvir Singh, and Divya Khurana, "Energy Optimization in Wireless Communication Network through Renewable Energy Sources (RES)," Power Electronics (IICPE), 2012 IEEE 5 th India International Conference, Dec. 2012.

[5] X. Wang, A. V. Vasilakos, M. Chen, Y. Liu, and T. T. Kwon, "A Survey of Green Mobile Networks: Opportunities and Challenges," Journal Mobile Networks and Applications, vol. 17, no. 1, pp. 4-20, Feb. 2012.

[6] M. A. Marsan, G. Bucalo, A. D. Caro, M. Meo, and Y. Zhang, "Towards Zero Grid Electricity Networking: Powering BSs with Renewable Energy Sources," IEEE international Conference on Communications Workshops (ICC), pp. 59-601, 2013.

[7] S. Vadgama and M Hunukumbure, "Trends in Green Wireless Access Networks," IEEE International Conference on Communications Workshops (ICC), June 2011. 
[8] E. Altman, M. K. Hanawal, R. ElAzouzi, and S. Shamai, "Tradeoffs in Green Cellular networks," in SIGMETRICS Performance Evaluation, 2011, pp. 67-71.

[9] Z. Niu, Y. Wu, J. Gong, and Z. Yang, "Cell zooming for cost-efficient green cellular networks," IEEE Communications Magazine, vol. 48, no. 11, pp. 74-79, November 2010.

[10] K. Son, H. Kim, Y. Yi, and B. Krishnamachari, "Base station operation and user association mechanisms for energy-delay tradeoffs in green cellular networks," IEEE J. Sel. Areas Commun., vol. 29, no. 8, pp. 1525-1536, Sep. 2011.

[11] "Optimizing performance and efficiency of Pas in wireless base stations," White Paper, Texas Instruments, Feb. 2009.

[12] P. Nema, R. K. Nema, and S. Rangnekar, "PV-solar/wind hybrid energy for GSM/CDMA type mobile telephony base station," International Journal of Energy and Environment, vol. 1, Issue. 2, pp. 359-366, 2010.

[13] C. McGuire, M. Brew, F. Darbari, S. Weiss, and R. Stewart, "WindFi a renewable powered base station for rural broadband," in Proc. IWSSIP, Apr. 2012, pp. 265268.

[14] Y. Chia, S. Sun, and R. Zhang. "Energy cooperation in cellular networks with renewable powered base stations," IEEE Wireless Communications and Networking Conference (WCNC), pp. 2542-2547, 2013.

[15] M. A. Marsan, G. Bucalo, A. D. Caro, M. Meo, and Y. Zhang, "Towards Zero Grid Electricity Networking: Powering BSs with Renewable Energy Sources,” IEEE international Conference on Communications Workshops (ICC), pp. 59-601, 2013. 
[16] A. Kwasinski, "Telecommunications Outside Plant Power Infrastructure: Past Performance and Technological Alternatives for Improved Resilience to Hurricanes," in INTELEC, 2009, pp. 1-6.

[17] Yun Tiam Tan, and Daniel S Kirschen, "Impact on the Power System of a Large Penetration of Photovoltaic Generation," IEEE Power Engineering Society General Meeting, 2007.

[18] A. Murata, H. Yamaguchi, and K. Otani, "A method of estimating the output fluctuation of many photovoltaic power generation systems dispersed in a wide area," Elect. Eng. Japan., vol. 166, pp. 9-19, Mar. 2009.

[19] T. Hoff and R. Perez, "Modeling PV fleet output variability,” Solar Energy, 2011.

[20] Matthew Lave, Jan Kleissl, and Joshua S. Stein, "Wavelet-Based Variability Model (WVM) for Solar PV power Plants," IEEE Trans. Sustainable Energy.

[21] L. L. Bucciarelli, Jr., "Estimating loss-of-power probabilities of stand-alone photovoltaic solar energy systems," Solar Energy, vol. 32, pp. 205-209, 1984.

[22] J. Song, V. Krishnamurthy, A. Kwasinski, and R. Sharma, "Development of a Markov-chain-based energy storage model for power supply availability assessment of photovoltaic generation plants," IEEE Trans. Sustain. Energy, vol. 4, no. 2, pp. 491-500, Apr. 2013.

[23] J. P. Barton and D. G. Infield, "Energy storage and its use with intermittent renewable energy," IEEE Trans. Energy Convers., vol. 19, no. 2, pp. 441-448, Jun. 2004. 
[24] J. Song, R. Zhao, and A. Kwasinski, "Design considerations for energy storage power electronics interfaces for high penetration of renewable energy sources," in Proc. IEEE.

[25] T. Hoff and R. Perez, "Modeling PV fleet output variability," Solar Energy, 2011.

[26] R. Zamora and A. Srivastava, "Control for microgrids with storage: Review, challenge, and research needs," Renewable and Sustainable Energy Reviews, vol. 14, pp. 2009-2018, 2010.

[27] A. Kwasinki, "Technology Planning for Electric Power Supply in Critical Events Considering a Bulk Grid, Backup Power Plants, and Micro-Grids," Systems Journal, IEEE, vol. 4, no. 2, pp. 167-178, June 2010.

[28] A. Kwasinski, V. Krishnamurthy, J. Song, and R. Sharma, "Availability Evaluation of Micro-Grids for Resistant Power Supply During Natural Disasters," Smart Grid, IEEE Transactions on, vol. 3, no. 4, pp. 2007- 2018, Dec. 2012.

[29] D. Robinson, D. Arent, and L. Johnson, "Impact of distributed energy resources on the reliability of critical telecommunications facilities," in Por. Int. Telecommunications Energy Conf. (INTELEC), 2006, pp. 1-7.

[30] A. Kwasinski, "Effects of Notable Natural Disasters from 2005 to 2011 on Telecommunications Infrastructure: Lessons from On-Site Damage Assessments,” in INTELEC, 2011, pp. 1-9.

[31] A. Kwasinski, and A. Tang. "Telecommunications Performance in the M=9.0 Offshore East Coast of Japan Earthquake and Tsunami, March, 2011," in Japan Society 
of Civil Engineers' International Symposium on Engineering Lessons Learned from the Giant Earthquake, Tokyo, Japan, 2012.

[32] A. Kwasinski, W. W. Weaver, P. L. Chapman, and P. T. Krein, "Telecommunications power plant damage assessment for hurricane katrina—Site survey and follow-up results," IEEE Syst. J., vol. 3, no.3, pp. 277-287, Sep. 2009.

[33] NOAA [Online]. Available: http://www.noaa.gov.

[34] J. Garche and A. Jossen, "Battery management systems (BMS) for increasing battery life time," in Proc. 3rd Int. Conf. Telecommunications Energy Special, Dresden, Germany, May 2000, pp. 81-88.

[35] K. W. E. Cheng, B. P. Divakar, H. Wu, K. Ding, and H. F. Ho, "Battery management system (BMS) and SOC development for electrical vehicles," IEEE Trans. Veh. Technol., vol. 60, no. 1, pp. 76-88, Jan. 2011.

[36] H. T. Lin, T. J. Liang, and S. M. Chen, "Estimation of battery state of health using probabilistic neural network,” IEEE Trans. Ind. Informat., vol. 9, no. 2, pp. 679685, May 2013.

[37] M. Chen and G. A. Rincon-Mora, "Accurate electrical battery model capable of predicting runtime and I-V performance," IEEE Trans. Energy Convers., vol. 21, no. 2, pp. 504-511, June 2006.

[38] S. Drouilhet and B. L. Johnson, "A battery life prediction method for hybrid power applications," U.S. Dept. Energy, Chicago, IL, Contract DE-AC36-83CH10093, Jan. 1997. 
[39] D. Salomonsson, L. Soder, and A. Sannino, “An Adaptive Control System for a DC Microgrid for Data Centers," IEEE Trans. Industry Applications, vol. 44, no. 6, pp. 1910-1917, Nov. 2008.

[40] J. Mitra, and M. R. Vallem, "Determination of Storage Required to Meet Reliability Guarantees on Island-Capable Microgrids with Intermittent Source," IEEE Trans. Power Systems, vol. 27, no. 4, pp. 2360-2367, Nov. 2012.

[41] G. M. Masters, Renewable and Efficient Electric Power Systems, Hoboken, NJ: Wiley, 2004.

[42] WeatherSpark

[Online].

Available: http://www.weatherspark.com/averages/29684/Austin-Texas-United-States accessed: $31^{\text {st }}$ Mar 2014.

[43] C. Chen, S. Duan, T. Cai, and B. Liu, "Online 24-h solar power forecasting based on weather type classification using artificial neural network," Solar Energy, vol, 85, no. 11, pp. 2856-2870, 2011.

[44] P. Poggi, G. Notton, M. Muselli, and A. Louche. Stochastic study of hourly total solar radiation in Corsica using a Markov model. International Journal of Climatology, Volume 20, Issue 14, Pages 1843-1860, 2000.

[45] J. Shi, W. J. Lee, Y. Liu, Y. Yang, W. Peng, "Forecasting Power Output of Photovoltaic System Based on Weather Classification and Support Vector Machines," in IEEE Transactions on Industry Applications, vol. 48, no. 3, pp. 1064 1069, Jun. 2012 
[46] A. Kwasinski and A. Kwasinski, "Architecture for green mobile network powered from renewable energy in microgrid configuration,” in Proc. IEEE WCNC, Shanghai, China, Apr. 2013, pp. 1273-1278.

[47] G. Auer.; et al.; "Energy Efficiency Analysis of the Reference Systems, Areas of Improvements and Target Breakdown," Energy Aware Radio and network tecHnologies (EARTH) Project deliverable D2.3, document INFSO-ICT-247733 EARTH, 2010.

[48] A. Kwasinski and A. Kwasinski, "Role of energy storage in a microgrid for increased use of photovoltaic systems in wireless communication networks," Telecommunication Energy Conference (INTELEC), 2014 IEEE $36^{\text {th }}$ International, IEEE, 2014.

[49] Charles J. Hanley, Georgianne H. Peek, and John D. Boyes, "Solar Energy Grid Integration System-Energy Storage (SEGIS-ES)," Sandia National Lab., Albuquerque, NM, and Livermore, CA, Rep. SAND2008-4247, Jul. 2008.

[50] The magazine of technology insiders IEEE spectrum, IEEE, Dec. 2012, pp. 14.

[51] J. P. Barton and D. G. Infield, "Energy storage and its use with intermittent renewable energy," IEEE Trans. Energy Convers., vol. 19, no. 2, pp. 441-448, Jun. 2004

[52] V. Kostylev and A. Pavlovski, "Solar power forecasting performance - towards industry standards," in $1^{\text {st }}$ International Workshop on the Integration of Solar Power into Power System, Aarhus, Denmark, Oct. 2011. 
[53] P. Fu and P. Rich, "A geometric solar radiation model and its applications in agriculture and forestry," Computers and Electronics in Agriculture, vol. 364, pp. 311-327, Jan. 2009.

[54] A. C. Harvey, Forecasting, Structural Time Series Models and the Kalman Filter, Cambridge University Press, 1989.

[55] G. Reikard, "Predicting solar radiation at high resolutions: A comparison of time series forecasts," Solar Energy, vol. 83, no. 3, pp. 342-349, March 2009.

[56] A. Mellit and A. Massi Pavan, "A 24-h forecast of solar irradiance using artificial neural network: Application for performance prediction of a grid-connected PV plant at Trieste, Italy," Solar Energy, vol. 84, no. 6, pp. 807-821, May 2010.

[57] A. Sfetsos, A. H. Coonick, "Univariate And Multivariate Forecasting of Hourly Solar Radiation with Artificial Intelligence Techniques," Solar Energy, vol. 68, no.2, pp. 169-178, 2000.

[58] Y. Kemmoku, S. Orita, and S. Nakagawa, "Daily insolation forecasting using a multi-stage neural network," Solar Energy, vol. 66, no. 3, pp. 193-199, Jun. 1999.

[59] T. Mitchell, Machine Learning, $1^{\text {st }}$ ed., McGraw-Hill Science/Engineering/Math, 1997, pp. 177-184.

[60] W. Hrdle, Smoothing Technique: With Implementation in S. New York: SpringerVerlag, 1991.

[61] M. H. DeGroo and M. J. Schervish, "Probability and Statistics," Reading, MA: Addison Wesley, 2001. 
[62] J. S. Armstrong and F. Collopy, "Error measures for generalizing about forecasting methods: Empirical comparisons," Int. J. Forecast., vol. 8, pp. 69-80, 1992.

[63] G. Papaefthymiou and B. Klockl, "MCMC for Wind Power Simulation," IEEE Transactions on Energy Conversion, vol. 23, no. 1, pp. 234-240, March 2008.

[64] A. Shamshad, M. A. Bawadi, W.M.A. Wan Hussin, T.A. Majid, and S.A.M. Sanusi, "First and second order Markov chain models for synthetic generation of wind speed time series," Energy, vol. 30, no. 5, pp. 693-708, 2005.

[65] A. Kwasinski and A Kwasinski, "Operational aspects and power architecture design for a microgrid to increase the use of renewable energy in wireless communication networks", in Proc. IEEE the International Power Electronics Conference (IPEC) ECCE-Asia, 2014.

[66] A. Kwasinski, "Lessons from Field Damage Assessments about Communication Networks Power Supply and Infrastructure Performance during Natural Disasters with a focus on Hurricane Sandy," FCC Wksp. Network Resiliency, Feb. 2013, New York City, NY, USA.

[67] A. Kwasinski, "Field technical surveys: an essential tool for improving critical infrastructure and lifeline systems resiliency to disasters," to be presented at the 2014 IEEE Global Humanitarian Technology Conference, San Jose, California.

[68] H. Bindner, T. Cronin, P. Lundsager, J. F. Manwell, U. Abdulwahid, and I. BaringGould, "Lifetime modeling of lead acid batteries," Riso Nat. Lab., Roskilde, Denmark, Apr. 2005. Riso Rep.

[69] U.S.Battery [Online]. Available: http://www.usbattery.com 
[70] S. D. Downing, and D. F. Socie, "Simple rainflow counting algorithms," International Journal of Fatigue, pp. 31, Jan. 1982.

[71] T. Lambert, P. Gilman, and P. Lilienthal, Micropower System Modeling with Homer. John Wiley and Sons, Inc., 2006, pp. 379-418. 


\section{Vita}

Youngsung Kwon was born in Seoul, Korea (Republic of Korea) in 1982, to Myungsik Kwon and Aewall Kang. He received his B.S. in Electrical Engineering from Soongsil University, Seoul, South Korea in 2009. He was accepted to the graduate program in Fall of 2010 and received the M.S.E. degree from the department of Electrical and Computer Engineering at The University of Texas at Austin on May 2012. During his doctoral studies, his primary research interests were focused on design of renewable energy sources, energy storage, and power output management algorithms for their operation in Microgrids.

Email: youngsung.kwon@utexas.edu

This dissertation was typed by the author. 